\title{
Development of an Equivalent Circuit of a Large Power System for Real- time Security Assessment
}

\author{
By \\ Gayan Wijeweera

\begin{abstract}
A thesis submitted to
the Faculty of Graduate Studies

in partial fulfilment of

the requirements for the degree of

Doctor of Philosophy
\end{abstract} \\ Department of Electrical and Computer Engineering \\ Faculty of Engineering \\ University of Manitoba \\ Winnipeg, Manitoba
}

October 2016

(C) Copyright

2016, D. G. P. Wijeweera 


\begin{abstract}
More and more system operators are interested in calculating transfer capability in realtime using real-time power flow models generated from the Energy Management System (EMS). However, compared to off-line study models, EMS models usually cover only a limited portion of the interconnected system. In most situations, it is not practical to extend the EMS model to capture the impact of the external systems and therefore using an equivalent network becomes necessary.
\end{abstract}

The development of equivalent circuits to represent external areas was a topic discussed over the last 50 years. Almost all of these methods require impedance information about the external area to develop the equivalent circuit. Unfortunately utilities do not have the external impedance information in the real-time. Therefore, normal industry practice is to use off-line studies to develop an equivalent circuit and use that circuit in the real-time operation without any validation. This can result in errors in the security assessment. Therefore, power industry need a method to develop or validate an equivalent circuit based on the available real-time information. This thesis work is focussed on meeting that industry need.

The work on this thesis presents two new methods that can be used to generate an equivalent circuit based on the boundary conditions. This method involves calculating 
equivalent impedance between two areas based on the boundary stations voltages, voltage angles and power leaving the boundary stations into external areas.

This thesis uses power system simulation between two areas to change the system condition to obtain different boundary bus voltages, voltage angles and power injections to generate necessary data. Regression analysis and least square method is then used to generate the equivalent circuit using these data. It is expected that system changes will provide necessary information in the real-time to generate the equivalent circuit.

The proposed methodology is validated with modified three area 300 bus system as well as using Manitoba Hydro's system. Contingency analysis, transfer level calculation and PV curves analysis is used to validate the proposed method. Simulation results show that the proposed method produces adequate accuracy in comparison with detailed off-line system models.

The main advantage of the proposed method as compared to other existing methods such as Ward and REI is that the proposed method does not require external impedance information to generate the equivalent circuit. The ability to generate reasonably good equivalent circuit only using available boundary information will help utilities to generate or validate the equivalent circuit based on the current system conditions, which will intern help improve the accuracy of the security assessment. 


\section{Acknowledgments}

I would like to express my appreciation and sincere thanks to Udaya Annakkage, my research advisor, for his guidance, support and advice throughout this research. I was truly enriched by the many discussions we had over the course of this work and look forward to continued collaboration.

I would like to thank Professor Athula Rajapakse for acting as my co-advisor in my M.Sc. and Ph.D. work as well as introducing me to Professor Cyrus Shafai as graduate student. I am grateful to Professor Ahmed Shalaby for acting as committee member during $\mathrm{Ph} . \mathrm{D}$. studies and providing valuable comments and advice.

I would like to thank Professor Cyrus Shafai for accepting me as one of his graduate student in September 2005 and all the help, guidance and life lessons provided to me during my early days in Canada.

I would like to express my sincere gratitude to my supervisor Dr. Wenjie Zhang

for numerous long discussions we had and various guidance and support provided to make my thesis a success. 
I am grateful to my employer, Manitoba Hydro, for financially supporting my studies and my supervisors Allan Silk, Michelle Rheault and Maria Neufeld for all the support provided to me during my studies.

I am very much grateful to Lisa Ruchkall and Chris Beaudoin for going through my thesis and providing valuable comments to improve my writing.

I wish to thank my parents for their continued love and support. Last but not least I would like to thank my wife Gayani for numerous sacrifices she made to support my education and raising our children Gavin, Galen and Garren. 


\section{Dedication}

To all the men and women working hard to make the world a better place. 


\section{Contents}

Front Matter

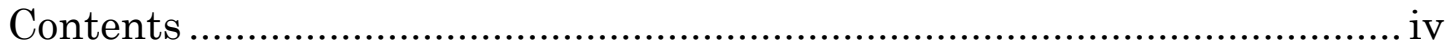

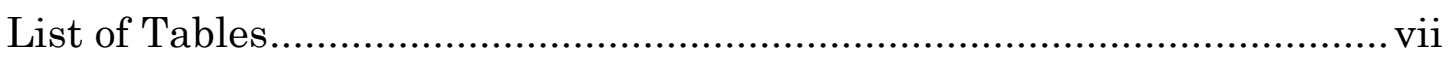

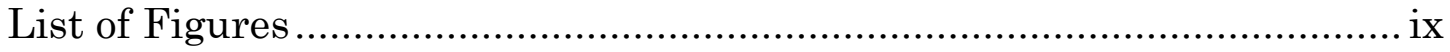

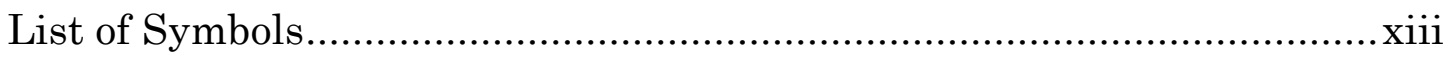

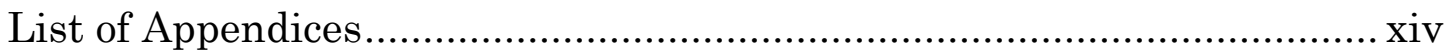

1 Introduction 1

1.1 Power System Control Centre ......................................................... 1

1.2 Situational Awareness and Security Assessments in Power Systems 4

1.2.1 Real-time Security Assessment ............................................... 7

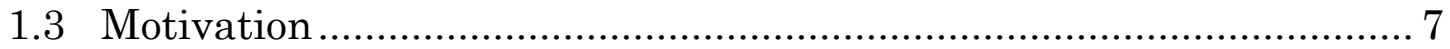

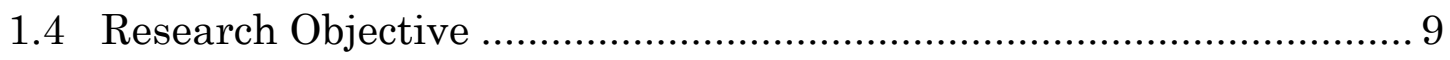

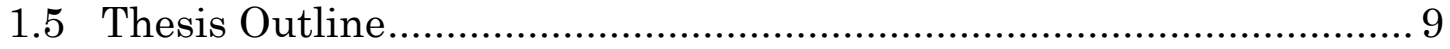

2 Developing an Equivalent Circuit $\quad 11$

2.1 Modelling Power System for Real-time Security Assessments ......... 11

2.2 Modelling the External Network Data in Real-time Security

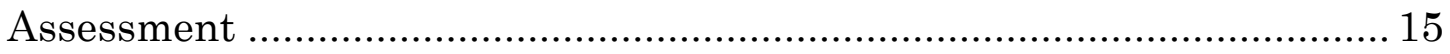

2.2.1 Use of Unreduced Power System Model for the External

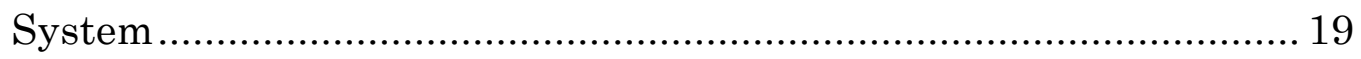

2.3 Use of an Equivalent Network to Represent the External System ... 22 
2.3.1 Evolution of Equivalent Circuits over Time ............................ 23

2.4 Main Methods Used in Developing Equivalent Circuits ................... 28

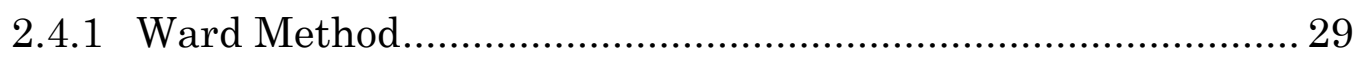

2.4.2 Ward PV Method.................................................................. 30

2.4.3 Extended Ward Method .......................................................... 30

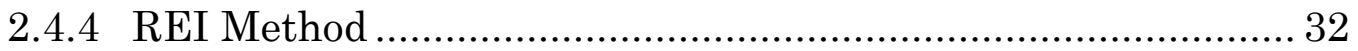

2.4.5 Adaptive Reduction Method ................................................. 35

2.4.6 External Network for Optimal Power Flow Applications ....... 36

2.4.7 PTDF Based Power System Equivalents .................................. 38

2.4.8 Equivalent Network for Policy Analysis ................................... 41

2.4.9 Reduced Network model for Fast Nodal Price Calculation..... 42

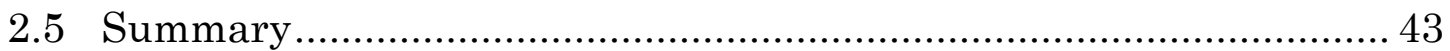

3 Development of an Equivalent Circuit using Linear Regression Method 45

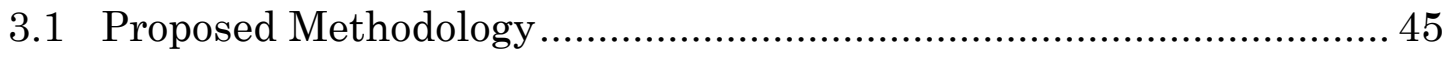

3.2 Developing Equivalent Circuit for Extended 300 bus system ........... 48

3.2.1 Boundary Matching and Model Merging ................................ 59

3.2.2 Validating Equivalent Circuit .................................................. 63

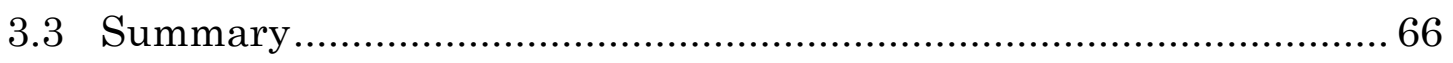

4 Development of an Equivalent Circuit using Least Square Method 67

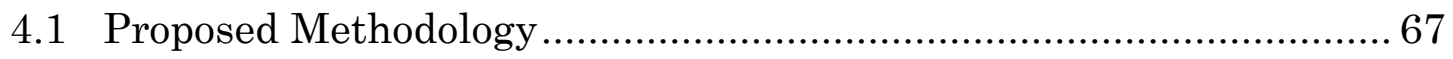

4.2 Developing an Equivalent Circuit...................................................... 71

4.2.1 Applying Additional Constraints ............................................. 76

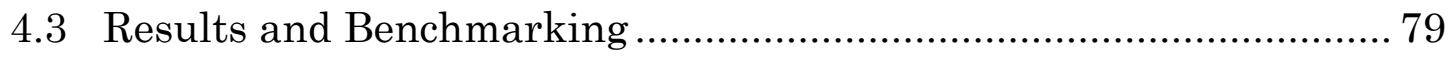

4.3.1 Contingency Analysis ............................................................. 79

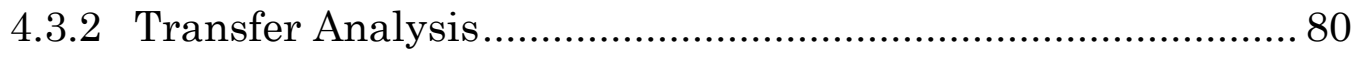

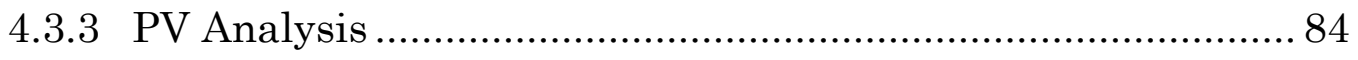

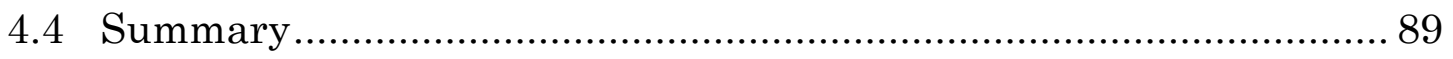


5 Model Validation with Manitoba Hydro System 90

5.1 Introduction to Manitoba Hydro …………........................................ 90

5.2 Manitoba Hydro Power System Model .............................................. 91

5.3 Creating Equivalent Circuit for Manitoba Hydro System ................. 98

5.4 Benchmarking the Equivalent Circuit ........................................... 101

5.4.1 Contingency Analysis ............................................................ 101

5.4.2 Use of Equivalent Circuit in Transfer Calculation ............... 115

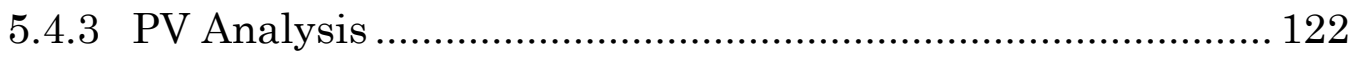

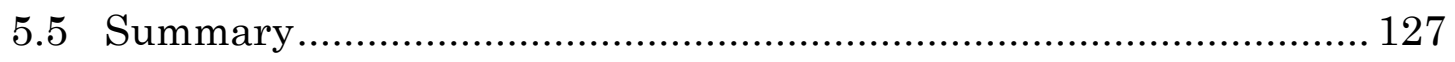

6 Proposed Improvements to Real-time Limit Calculation Process 128

6.1 Contingency Analysis Associated with Load and Generation Loss. 128

6.2 System Response during Contingency Analysis ............................. 135

6.3 Compensation for Reduced Generation and Loads in the Cut-off Area 139

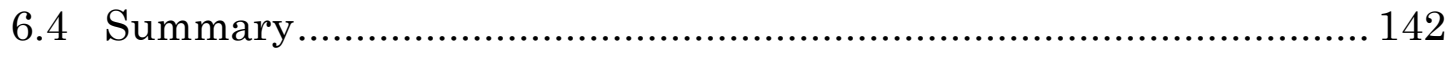

7 Conclusions and Future Work 143

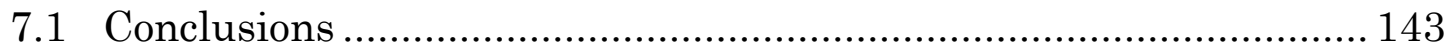

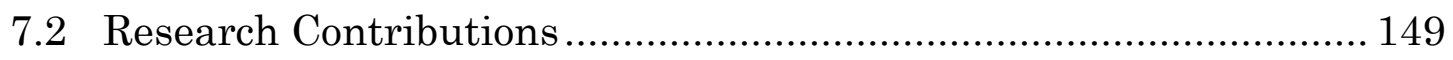

7.3 Future Work ……………......................................................... 150

$\begin{array}{ll}\text { Back Matter } & 153\end{array}$

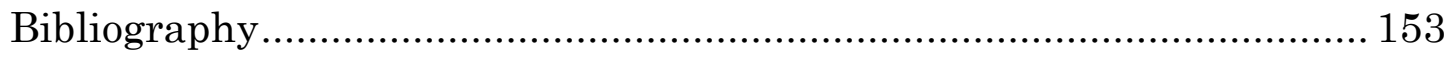

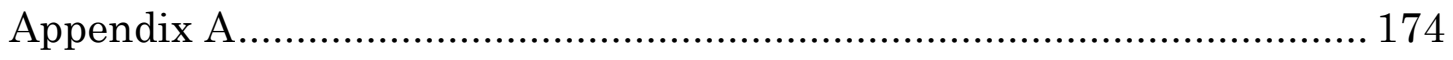




\section{List of Tables}

Table 1: Active Power Flow Across Various Interfaces and Paths ............................... 53

Table 2: Equivalent Circuit Parameters and correlation coefficient .............................. 59

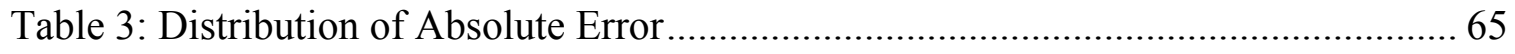

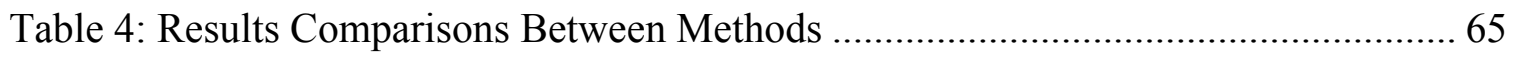

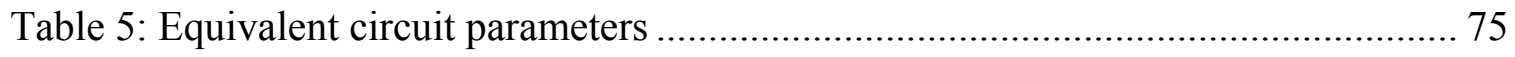

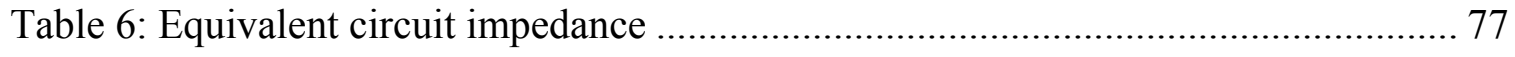

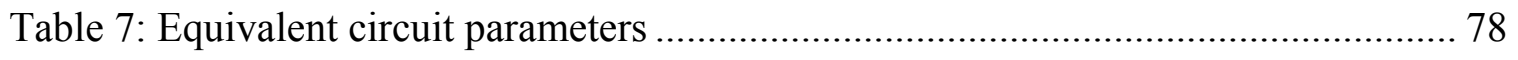

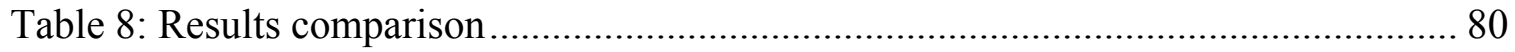

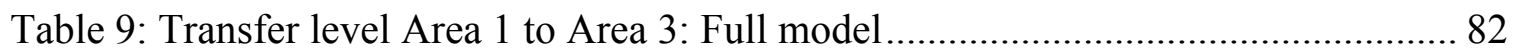

Table 10: Transfer level Area 1 to Area 3: Reduced Model......................................... 83

Table 11: Transfer level Area 1 to Area 2: Full model ................................................. 83

Table 12: Transfer level Area 1 to Area 2: Reduced Model........................................... 83

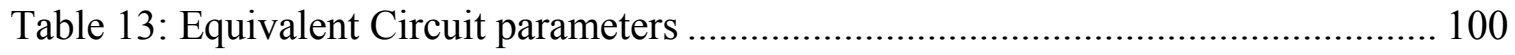

Table 14: Pre Contingency Flow on Selected Lines ............................................... 102

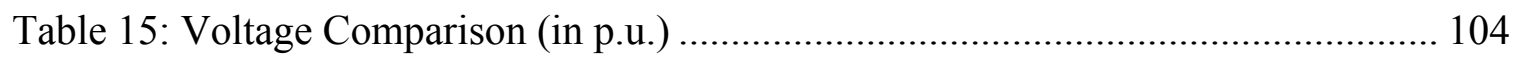

Table 16: Interface Active Power Comparison (MW) ............................................. 106

Table 17: Interface Reactive Power Comparison (MVAr) ...................................... 108

Table 18: Active Power Comparison Along the Lines (MW) ................................... 110 
Table 19: Line Reactive Power Comparison (MVAr) .............................................. 112

Table 20: Phase Shifter Angle Comparison (Degree)............................................. 114

Table 21: Transfer Level Comparison between Two Models ((MW) .......................... 120 


\section{List of Figures}

Figure 1: Internal System External System and Buffer Zone........................................... 16

Figure 2: Part of the Interconnected System............................................................. 17

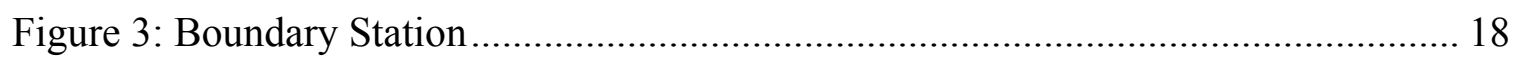

Figure 4: Modelling Cut-off Lines in Boundary Stations .............................................. 18

Figure 5: North American Interconnection Regions....................................................... 21

Figure 6: Step1-Identify the Group of Buses Connected to Boundary ............................. 33

Figure 7: Step2-Adding Bus Injection in the Group to a Fictitious Node ........................ 33

Figure 8: Step3-Use Gaussian Elimination to Remove all the Buses in the Designated

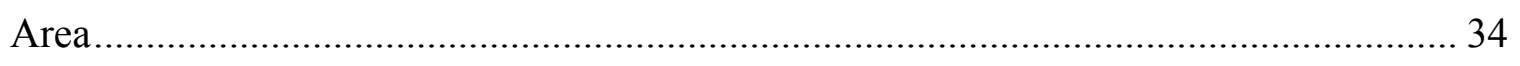

Figure 9: Part of the Interconnected System................................................................. 46

Figure 10: Estimating Equivalent Circuit Parameters ………………………………...... 47

Figure 11: Three area 300 Bus System ..................................................................... 50

Figure 12: Three Area System with Added External Area ............................................... 51

Figure 13: Plot of Power Flow into Boundary Station with Voltages and Angle Difference Multiplication for $138 \mathrm{kV}$ Boundary Station ............................................... 56

Figure 14: Plot of Power Flow into Boundary Station with Voltages and Angle Difference Multiplication for $230 \mathrm{kV}$ Boundary Station ............................................... 56 
Figure 15: Plot of Power Flow into Boundary Station with Voltages and Angle

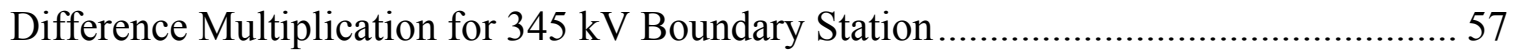

Figure 16: Four Area System with Equivalent Circuit ............................................ 58

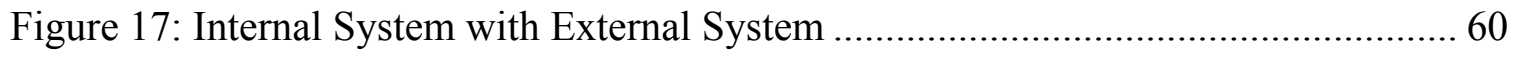

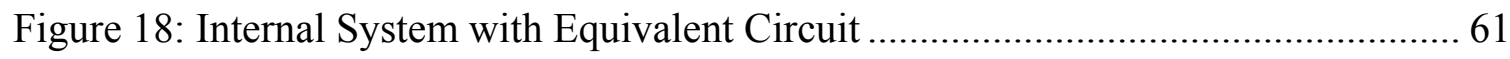

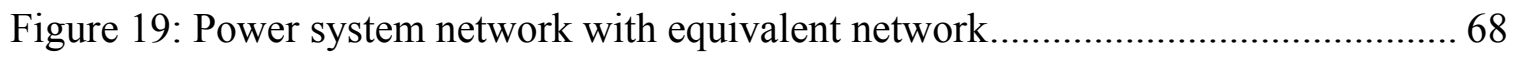

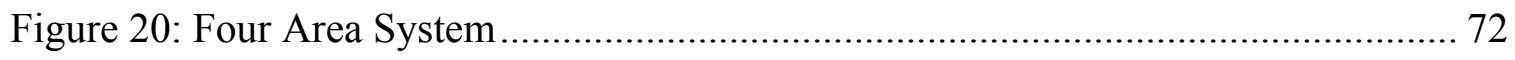

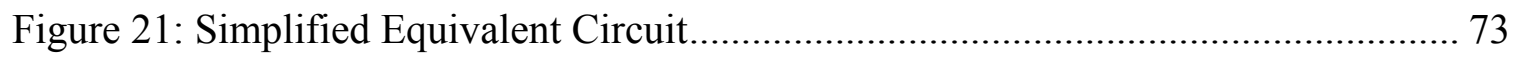

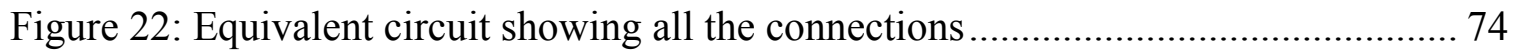

Figure 23 : Equivalent circuit connections ...................................................... 78

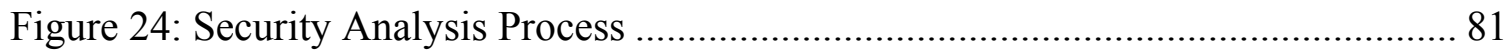

Figure 25: Pre Contingency voltage variation of bus 34 with transfer level .................. 85

Figure 26: Post Contingency voltage variation of bus 34 with transfer level.................. 85

Figure 27: Pre Contingency voltage variation of bus 45 with transfer level .................. 86

Figure 28: Post Contingency voltage variation of bus 45 with transfer level.................. 86

Figure 29: Pre Contingency voltage variation of bus 60 with transfer level .................. 87

Figure 30: Post Contingency voltage variation of bus 60 with transfer level................. 87

Figure 31: Pre Contingency voltage variation of bus 562 with transfer level ................ 88

Figure 32: Post Contingency voltage variation of bus 562 with transfer level................ 88

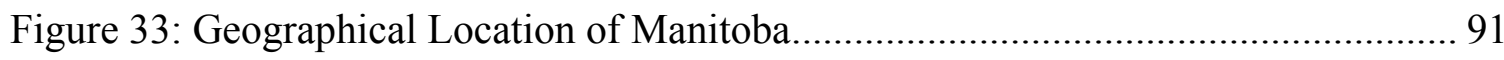

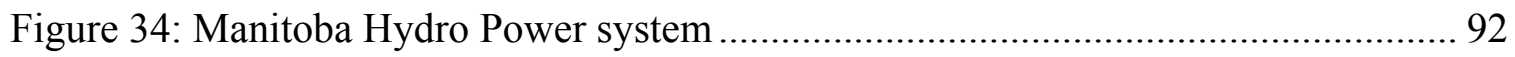

Figure 35: Modelling boundary buses in the EMS model. Actual system (above) and how

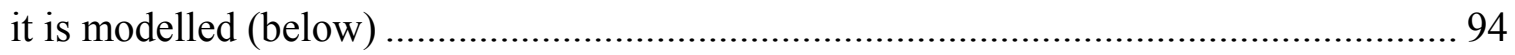




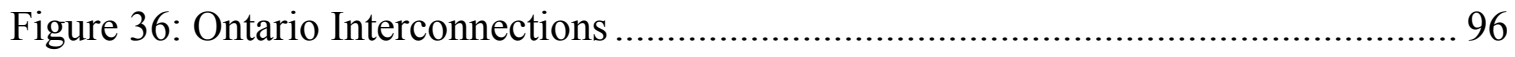

Figure 37: Flow Change on $500 \mathrm{kV}$ line and East-West tie lines.................................... 97

Figure 38: Flow change on East-West tie line for Loss of $500 \mathrm{kV}$ Line in Manitoba...... 98

Figure 39: Location of Selected Boundary Stations in the USA ……............................ 99

Figure 40: Lines Selected for Contingency Analysis ................................................... 102

Figure 41: Transfer Limit Calculation Process ......................................................... 116

Figure 42: Transfer level Calculation Process for Prior Outage Condition.................... 119

Figure 43: PV Analysis for Major Manitoba Stations. Variation of bus voltage for Station

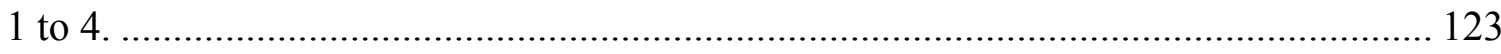

Figure 44: PV Analysis for Major Manitoba Stations. Variation of bus voltage for Station

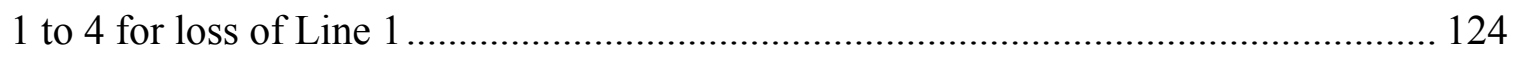

Figure 45: PV Analysis for Major Manitoba Stations. Variation of bus voltage for Station

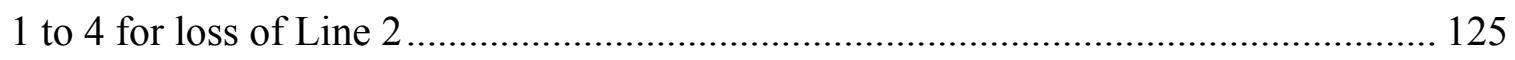

Figure 46: PV Analysis for Major Manitoba Stations. Variation of bus voltage for Station

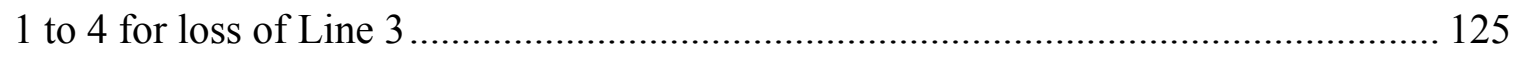

Figure 47: PV Analysis for Major Manitoba Stations. Variation of bus voltage for Station

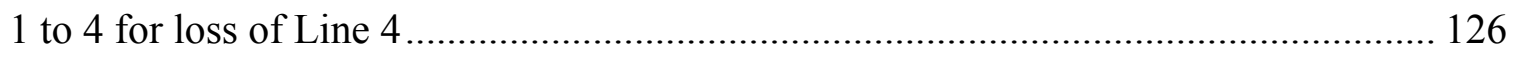

Figure 48: PV Analysis for Major Manitoba Stations. Variation of bus voltage for Station

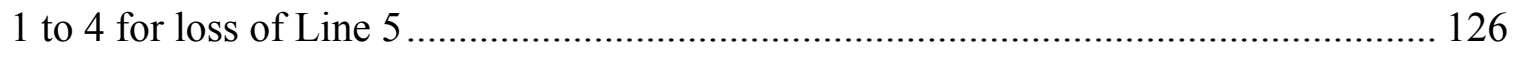

Figure 49: PV Analysis for Major Manitoba Stations. Variation of bus voltage for Station

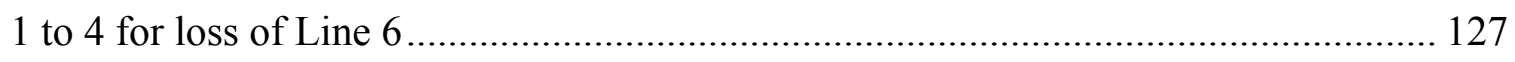

Figure 50: Change of Manitoba Hydro Total Export with a loss of industrial customer 132

Figure 51: Change of Manitoba Hydro total export with loss of a tie line ..................... 133 
Figure 52: Algorithm to simulate both governor and AGC response.......................... 135

Figure 53: Proposed Real-time security assessment method ..................................... 137

Figure 54: Part of the Interconnected System........................................................ 140

Figure 55: Proposed Equivalent system.............................................................. 141 


\section{List of Symbols}

$\mathrm{n} \quad$ Number of nodes in the power system

$\mathrm{Y}_{\mathrm{ii}} \quad$ Self admittance of the node $\mathrm{i}$

$Y_{i j} \quad$ Mutual admittance of the node $i$ and $j$

$\mathrm{V}_{\mathrm{i}} \quad$ Phasor voltage of the node $\mathrm{i}$

$\mathrm{I}_{\mathrm{i}} \quad$ Phasor current flowing intothe network at node $\mathrm{i}$

$\mathrm{P}_{\mathrm{i}} \quad$ Active power at node $\mathrm{i}$

$\mathrm{Q}_{\mathrm{i}} \quad$ Reactive power at node $\mathrm{i}$ 


\section{List of Appendices}

Appendix A PSSE file used to modify three area 300 bus system 


\section{Chapter 1}

\section{Introduction}

A reliable power supply is the expectation of every customer connected to the electric grid and it is also the vision of every power utility. This can be achieved since every major power grid is monitored and controlled in real-time by the system operators located in power system control centres. This Chapter provides an introduction to power system control centres, and the power system security assessment carried out by the control centres.

\subsection{Power System Control Centre}

The power system control centre can be considered as the central nerve system of the power system [1-4]. It is the duty and the responsibility of the system operators located in the control centre to monitor and operate the power system in most secure and reliable manner. 
Control centres are equipped with many tools to help system operators manage the power system. The main application used by every control centre is the Energy Management Systems (EMS's). An EMS consists of a collection of many tools that enable power system operators to carry out their functions in an efficient and effective manner.

A Supervisory Control and Data Acquisition (SCADA) system is one of the main components of an EMS [5-6]. SCADA systems bring system wide data into the control centre and send various control signals to remote devices/stations through dedicated communication systems. The data coming into control centre includes station voltage, active and reactive power flow on lines and equipment, status indication of circuit breakers and switches, and various alarms and warnings. This data is processed through the state estimator to correct any errors introduced due to measuring and data transmission and to estimate any missing data [7-12].

The meter values (once processed) are displayed in one of many display screens in the EMS. The power system may be displayed geographically or with electrical schematics. Various displays are provided for generation, transmission, and distribution systems.

Meter readings are also checked against various system operating limits. For example, line and transformer loadings are checked against equipment ratings while station voltages are checked against voltage criteria. When a facility is operating outside the desired operating range, warning alarms are created in the EMS for operators to take correc- 
tive actions. These operator actions may include switching reactors/capacitors, changing transformer taps, turning on and off generators, changing generator loading levels, and operating breakers. These functions may be carried out remotely from the control centres through SCADA or done at local stations.

Today, most area power systems are interconnected to form the regional or continental power grids for reliability and economic benefit. However, an area power system will be impacted by neighbouring power systems due to interconnections. Therefore, most area control centres are equipped with the EMS that has the ability to monitor the interconnected system beyond their functional control areas. Control centres monitoring the system beyond their control areas share real-time data through Inter-Control Centre Communications Protocol (ICCP) [13-15].

Most control centres also act as "Balancing Authorities" responsible for balancing generation with system load, export/import commitments, and operating reserve requirements within the control areas [16-23]. These functions include unit commitment and economic dispatch calculations [24-33] and Automatic Generation Control (AGC) control for variation in load, frequency and interchange schedule [34-40].

There are other auxiliary applications in today's control centre. Some of these applications include weather forecast, fire-monitoring, outage management, and transmission scheduling and tagging. 


\subsection{Situational Awareness and Security Assess- ments in Power Systems}

Situational Awareness (SA) is defined by M.R. Endsley as "the perception of the elements in the environment within a volume of time and space, the comprehension of their meaning and the projection of their status in the near future" [41, 42]. In simple terms, Situational Awareness is the ability to identify, process, and comprehend the critical components of information about what is happening and what is about to happen. The application of SA techniques can be traced back over 60 years ago in the United States Air Force [43]. Since then situational awareness and security assessment has been heavily adopted by air traffic controllers and nuclear power plants $[44,45]$.

Situational awareness and security assessment also find application in the power industry [46-49]. Today's power system control centres are equipped with various advanced situational awareness and security assessment tools to provide system operators with early warning and mitigation plans. The basic form of situational awareness in a control centre is to monitor facility operating conditions such as station voltages, loading levels, and so on, to compare against operating limits, and to generate display system statuses and alarm to draw operators' attention. This basic SA application ensures that line loading and bus voltages are operated within acceptable levels during normal system operation. 
The power system is not a static system. It is a dynamic system and vulnerable to various types of disturbances such as outage to a transmission line, power transformer, or generator. The system operator must always posture the system such that the loss of a single element from the current operating state does not jeopardize the reliability of the power system. In the power system industry, the potential loss of a contingent element is referred to as n-1 (n minus one) contingency.

The need for a security assessment tool, which can predict the stability of the power system after a disturbance, was first recognized by the United States Federal Energy Regulatory Commission (FERC) in its final report prepared for the North East blackout in 1965. The report recommended that utilities should have a security assessment tool in the control centre that is capable of periodically assessing the system security for selected key contingencies [50]. This tool is commonly known as Real-Time Contingency Analysis (RTCA) and is now part of the EMS used in most utilities [51-54]. RTCA simulates a set of pre-identified contingencies on the real-time power flow model. More details on creating real-time power flow model will be discussed in Chapter 2 . Alarms are generated when post-contingency equipment loading or voltage exceeds postcontingency operating limits. The alarms allow system operators to take proactive actions to ensure system stability in the event of any single pre-identified contingencies. This process of creating power flow models and performing contingency analysis runs periodically in a predetermined time interval to assess the system security in a continuous manner. The current industry practice for security assessment is to design and operate the power system to withstand a set of contingencies. 
With this approach, contingency analysis assesses power system security at the current operating state. In other words, RTCA addresses the question as to whether the current operating condition is secure or not. However, it cannot answer the question as to how secure the current operating condition is.

Traditionally System Operating Limits (SOL) are derived from the off-line operating studies. The engineers responsible for power system operations perform studies with off-line power system model to determine the transfer capabilities across various internal and external interfaces and establish any operating restrictions. These limits are then implemented in the EMS to provide operating limits to the operators. Since transfer capabilities are function of operating condition such as generation and loading conditions, it is impossible for the off-line studies to account for the all real-time operating conditions, for which the transfer capability is derived. As a result, more conservative assumptions are made to cover the worst operating conditions, which results in more restricted system operation.

Power utilities have experienced increased challenges in maintaining power system reliability over the years. Capital cost constraints compounded with the increased difficulty in acquiring a right of way to build new facilities due to environmental regulations, have forced system operators to look at new ways of maximizing the use of the existing power grid without compromising system security. One of the strategies that many power utilities have taken to address this challenge is the development of the real-time security assessment technology [55]. 


\subsubsection{Real-time Security Assessment}

North American Electric Reliability Corporation (NERC) defines real-time assessment as "An evaluation of system conditions using Real-time data to assess existing (pre-Contingency) and potential (post-Contingency) operating conditions. The assessment shall reflect applicable inputs including, but not limited to: load, generation output levels, known Protection System and Special Protection System status or degradation, Transmission outages, generator outages, Interchange, Facility Ratings, and identified phase angle and equipment limitations" [136]. Based on this definition, this thesis adopts the term "real-time security assessment" for security assessment performed using the real-time data. The term "online" for the real-time and "off-line" using non real-time data are also commonly used in the literature.

\subsection{Motivation}

As presented in Section 1.2, real time security analysis is based on the real-time power flow model generated from the EMS. Proper representation of external system is necessary for the accurate real time security assessment. The common practice is to use an equivalent circuit as developed in the offline studies to represent the external area in the real time security assessment.

Today, commercially available power system study applications can be used to automatically create an equivalent circuit using admittance reduction methods [150]. De- 
veloping equivalent circuits in the utility environment typically consists of the following steps:

- Create an off-line power flow model that represents the typical operation of the system.

- Identify the external area. Since real-time studies start from the power flow model generated from the utility's EMS, the external area for the real-time studies is the area not modelled in the utility's EMS.

- Use impedance reduction methods to generate the equivalent circuits for the external area.

- Model the equivalent circuits in the utility's EMS.

- Update the equivalent circuits using the state estimator to meet boundary conditions at the boundary of the buffer zone.

Clearly the equivalent circuit created with the above method is only valid for the original base case conditions. To account for different operating conditions, several equivalent circuits may be developed to represent various operating conditions such as export, import, high load, low load, summer, winter, etc.

The equivalent circuit is only accurate if the equivalent circuit impedance can be adaptive to the changes of impedance in operating condition of the external area. Unfortunately, utilities do not have real-time information of the external system, which represents a challenge in developing accurate equivalent circuit for real-time security assessment. 
The objective of the real-time analysis is to eliminate some of the conservative assumptions used in off-line studies so as maximize the use of the transmission system. Therefore, there is a dire need in the power system real-time security assessment to develop accurate equivalent circuits that are based on available real-time information, which is a new area of research in the industry today.

\subsection{Research Objective}

The objective of this thesis is to develop equivalent circuits using available realtime boundary information to represent the external area system for real-time security assessment. Real time boundary information such as boundary bus voltages, voltage angle and power injection from the external stations are commonly available from station metering such as Phasor Measurement Units (PMU). This thesis proposes two methods of deriving equivalent circuit to enhance the real time security assessment.

\subsection{Thesis Outline}

In this chapter background information of security assessments and power system control centres was introduced. It was shown that real-time security assessment plays a key role in the security of the power system. 
Chapter 2 presents details on how to present the power system in the real-time security assessments. Special attention is given to modelling external data in the security assessment.

Chapter 3 presents the proposed method to develop equivalent circuit based on the boundary information using linear regression method. The proposed method is validated with the modified IEEE three area 300 bus system.

Chapter 4 presents the improved method to generate equivalent circuit using least square method. This method is also validated using IEEE three area 300 bus system.

Chapter 5 introduces Manitoba Hydro system and present the work related to developing equivalent circuit for the Manitoba Hydro EMS system.

Chapter 6 proposes a few ways in which current security assessment can be improved. Particular attention will be given to the contingencies associated with generation and load losses as well as modelling various components in the power system during the contingency analysis.

Chapter 7 summarizes the study work in this thesis and presents suggestion for the future works. 


\section{Chapter 2}

\section{Developing an Equivalent Circuit}

This chapter describes details on modelling power system in the real-time security assessment. Special attention will be given to modelling external area in the security assessment.

\subsection{Modelling Power System for Real-time Secu- rity Assessments}

Most real-time security assessments in control centres start from the real-time power flow model. Therefore creating a power flow data model file which represents the actual operating state of the power system is very important. This section provides a very brief overview on the creation of a power flow model and the various equations associated with solving power flow model. Detailed discussion on power flow models can be found in the literature [56-58]. The network equation of the power system can be written in matrix form as: 


$$
\left[\begin{array}{c}
I_{1} \\
I_{2} \\
. . \\
I_{n}
\end{array}\right]=\left[\begin{array}{cccc}
Y_{11} & Y_{12} & . . & Y_{1 n} \\
Y_{21} & Y_{22} & . . & Y_{2 n} \\
. . & . . & . . & . . \\
Y_{n 1} & Y_{n 2} & . . & Y_{n n}
\end{array}\right]\left[\begin{array}{c}
V_{1} \\
V_{2} \\
. . \\
V_{n}
\end{array}\right]
$$

Where:

$\mathrm{n} \quad$ is the total number of nodes in the power system

Yii is the self admittance of the node $\mathrm{i}$

Yij is the mutual admittance of the node $i$ and $j$

$\mathrm{Vi} \quad$ is the phasor voltage of the node $\mathrm{i}$

Ii is the phasor current flowing into the network at node $\mathrm{i}$

If the current injections at all buses are known, the above equation will become a set of linear equations. The equations can be solved to find the voltage at each node. In practice, not all the current injections into the system are known. The relationship between current $\left(\mathrm{I}_{\mathrm{k}}\right)$ at any node $\mathrm{k}$, with active power $\left(\mathrm{P}_{\mathrm{k}}\right)$, reactive power $\left(\mathrm{Q}_{\mathrm{k}}\right)$, and voltage $\left(\mathrm{V}_{\mathrm{k}}\right)$ is given by the following formula (where $\mathrm{j}$ is the imaginary unit).

$$
I_{k}=\frac{P_{k}-j Q_{k}}{V_{k}}
$$

The four basic parameters associated with each bus in a practical power system are: active power $(\mathrm{P})$, reactive power $(\mathrm{Q})$, voltage magnitude $(\mathrm{V})$, and voltage angle $(\theta)$. Only some of the parameters are known for any given bus in the power system. Depend- 
ing on the known parameters, buses in the network can be divided into three different types:

- Load bus or PQ buses. These are the buses with loads where active and reactive power consumption is known. Voltage and voltage angle for these buses can be derived by solving the network equations.

- Generation buses or PV buses. These are the buses with generators where active power and voltage magnitude is known. These buses change their reactive power output to maintain the specified local or remote bus voltage. The network solution will provide the reactive power output for these buses and voltage angle.

- Swing bus or the slack bus. These are the buses where voltage magnitude and angle is specified. This is a special generator bus in the network and it is used to balance the active power output of the island in which it is connected. Therefore, active power out of this bus is initially unknown. It is necessary to solve the network equations to determine the active power and reactive power output of this bus. Voltage angle of the swing bus is generally fixed and the voltage angles of all other buses within the same island are calculated with respect to the swing bus voltage angle.

Every effort should be taken to ensure that the power flow model represents the actual system condition under investigation since security assessment starts with the power flow model. This includes formulating power flow model data from the actual system data. 
Two types of data are required to generate the power flow model. The first set of data is the network modelling data which includes the impedance of all the network elements connected to the power system under investigation. This data is used to generate the bus admittance matrix or the Y matrix. As explained previously, the power system model contains the buses from its own system and may include parts from an external system. The impedance values of the system's own network elements can be collected with some effort. Greater effort is required to collect the impedance value of the external system. It is also very important to maintain the accuracy of these network impedance values after various system improvements and changes. Once these data are collected, the admittance matrix can be generated based on the system configuration. It is important to find a mechanism to update this admittance matrix in real-time with planned and unplanned system outages for a real-time power flow model.

The second set of data necessary to generate the power flow model is the operating data which includes active and reactive power demand at PQ buses, and the active power generation and voltage magnitude of PV buses. Unlike network model data, these operating data could change from minute to minute.

It is necessary to generate a power flow model in real-time in order to perform real-time analysis. This requires utilities to update both the $\mathrm{Y}$ matrix and the operating data on a real-time basis. As mentioned in the previous section, most power systems are interconnected with each other. Therefore, utilities have to model and update the internal 
data together with an external area in real-time for the power flow data used in the realtime security assessments.

\subsection{Modelling the External Network Data in Real-time Security Assessment}

The method of modelling external data in the security assessment depends on the utility. Various utilities choose different methods to model the external area. This section describes various choices made by utilities.

Before going further, it is important to understand some terminology and concepts associated with internal areas and external areas. A system in which a utility has control over an area and in which the utility would like to perform the security analysis is generally known as the internal system. Any system outside the internal system is generally known as the external system. As explained before, the internal system can be impacted not only by the activities happening in the internal system (where the control centre has authority) but also can be impacted by the activities happing in the external system (where the control centre has no authority). Therefore, it is necessary to model the external system accurately in any security assessments or transfer calculation assessments. The impact of the external system on the internal system reduces with increasing electrical distance. Therefore, the requirement for detail is greater in the external area immediately adjacent to the internal area than it is for the rest of the external area. This boundary area is generally known as the buffer zone or is sometimes called the internal-external area. 
The remaining external system is called the remote section or outer-external system. The internal, external, buffer and remote systems are illustrated in Figure 1.

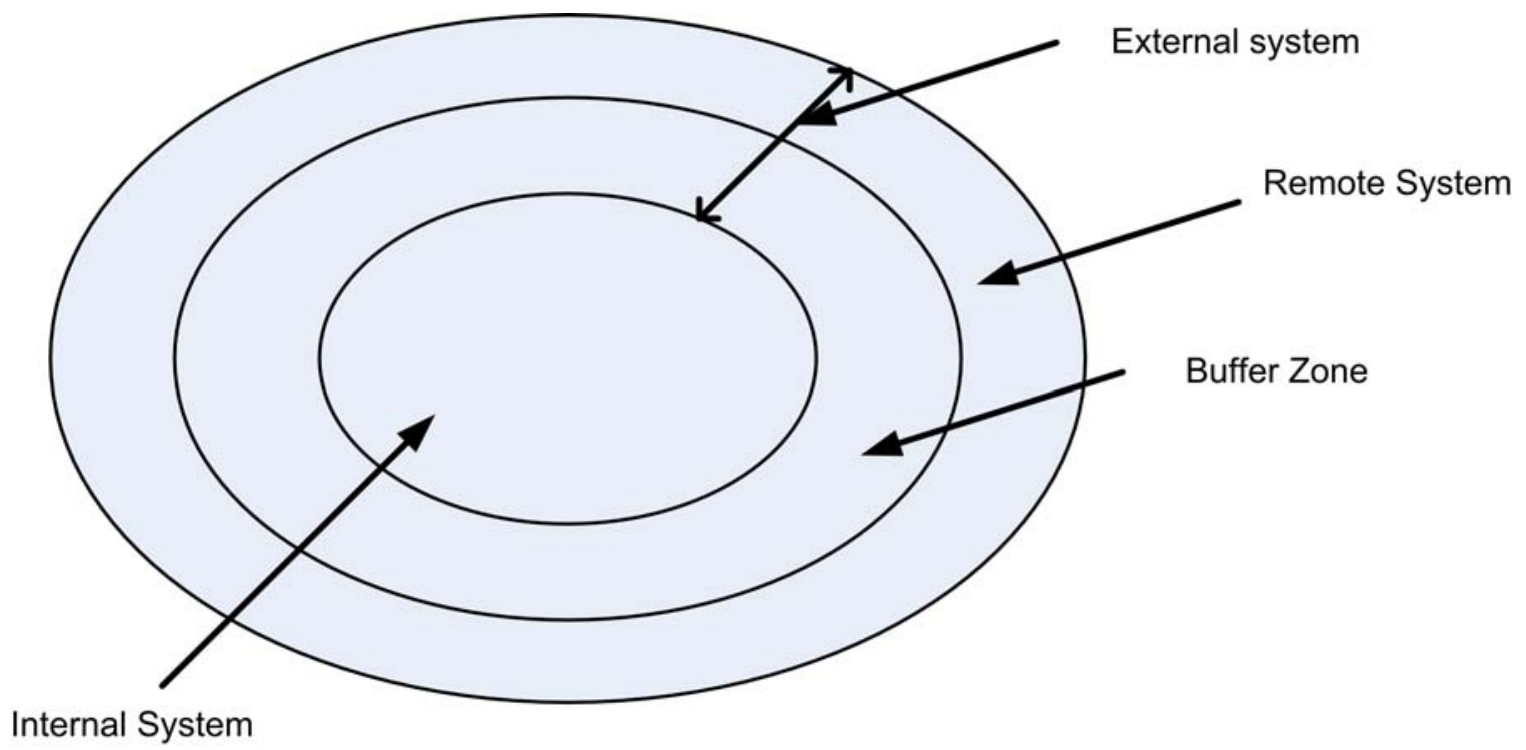

Figure 1: Internal System External System and Buffer Zone

Consider another example where part of the interconnection consists of Areas A, $\mathrm{B}, \mathrm{C}$ and $\mathrm{D}$ and tie lines connecting these areas as shown in Figure 2. Area-A is the entity interested in carrying out real-time transfer analysis and its EMS model contains all the major electrical equipments in Area-A as the internal system, and parts of Area-B, Area$\mathrm{C}$ and Area-D (as shown) as the buffer system. The station $\mathrm{C} 1$ is the boundary station in Area-C, i.e. it is at the boundary of the buffer system in Area-A's model. Similarly stations D1, D2, D3, and D4 are the boundary stations in Area-D and included in the Area-A EMS model. The rest of the area is the remote system. 


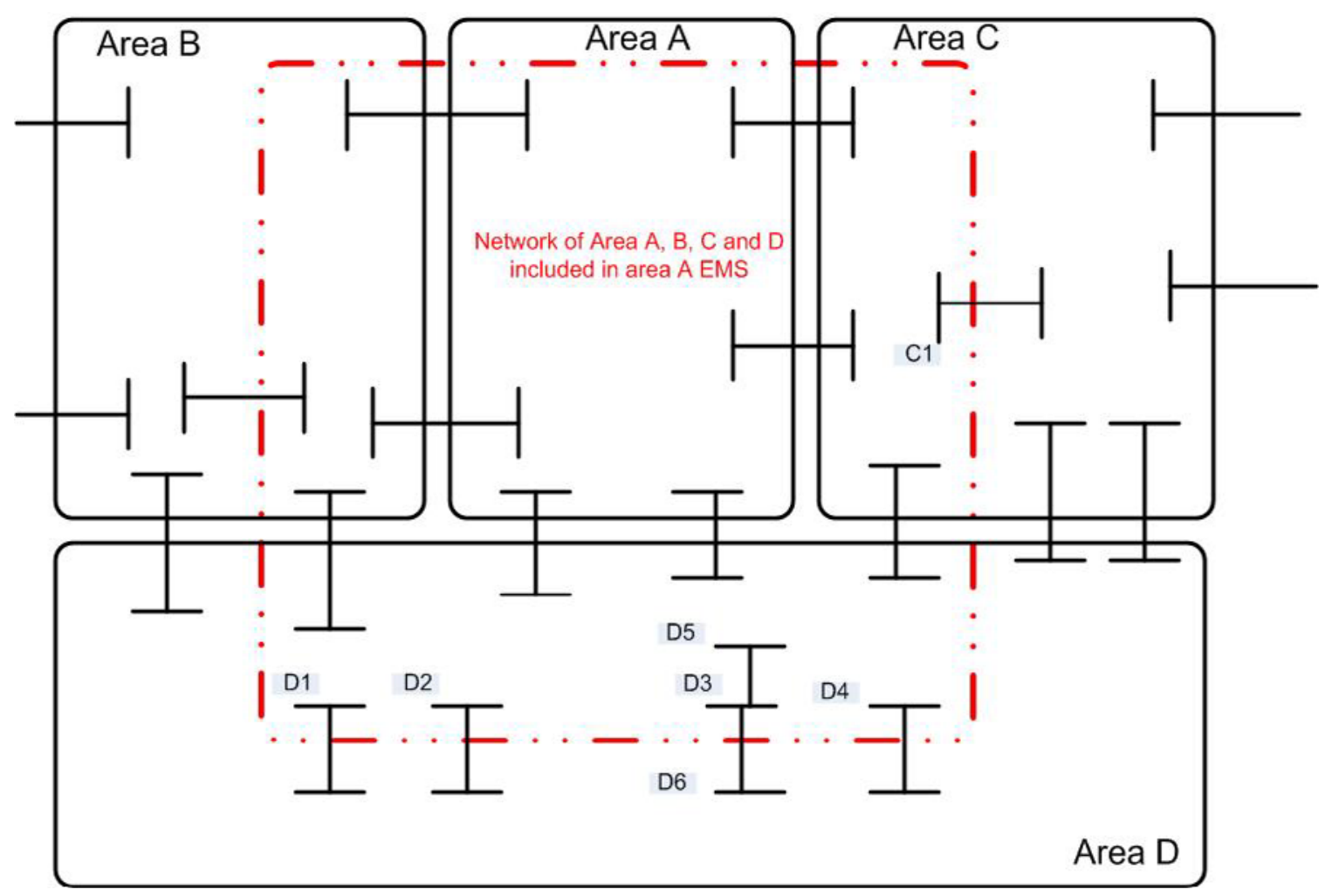

Figure 2: Part of the Interconnected System

Different utilities use different ways to represent the remote system in the security assessment. Some of these methods are:

- Ignore the remote system

- Use the full power system model

- Use an equivalent network

The easiest method is to ignore the remote system beyond the buffer zone. When using this method, most utilities model the cut off lines as a load at the boundary station. This is illustrated Figure 3 and Figure 4 for boundary station D3. Figure 3 shows the power system model for station D5, D3 and D6. The line AB is the boundary of the 
buffer zone. Station D3 is the boundary station, Station D6 is the cut off station and line between station D3 and D6 is the cut off line. Figure 4 shows the power flow between the stations D3 to D6 can be modelled in station D3 as a positive or negative load depending on the real-time power flow.

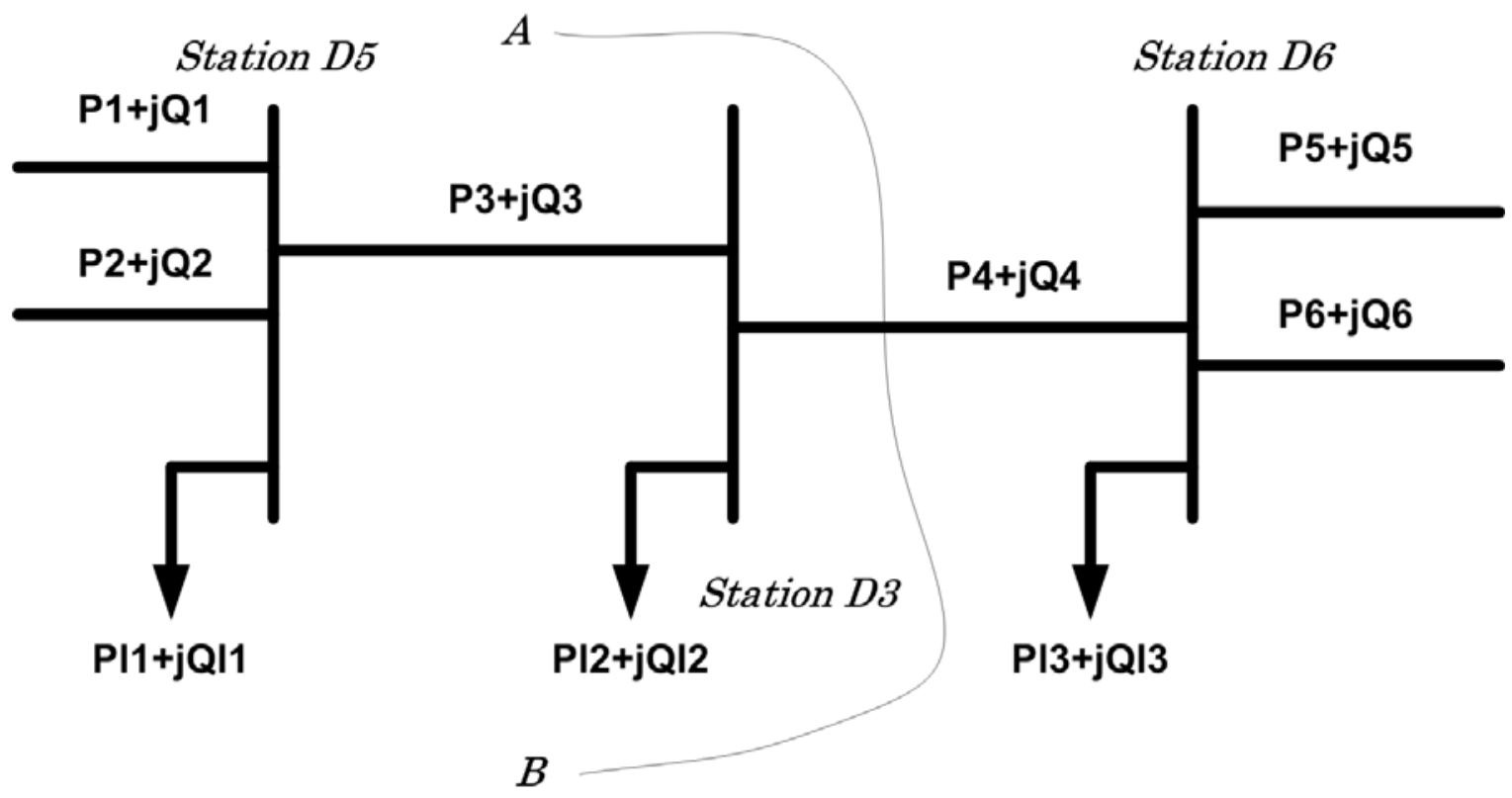

Figure 3: Boundary Station

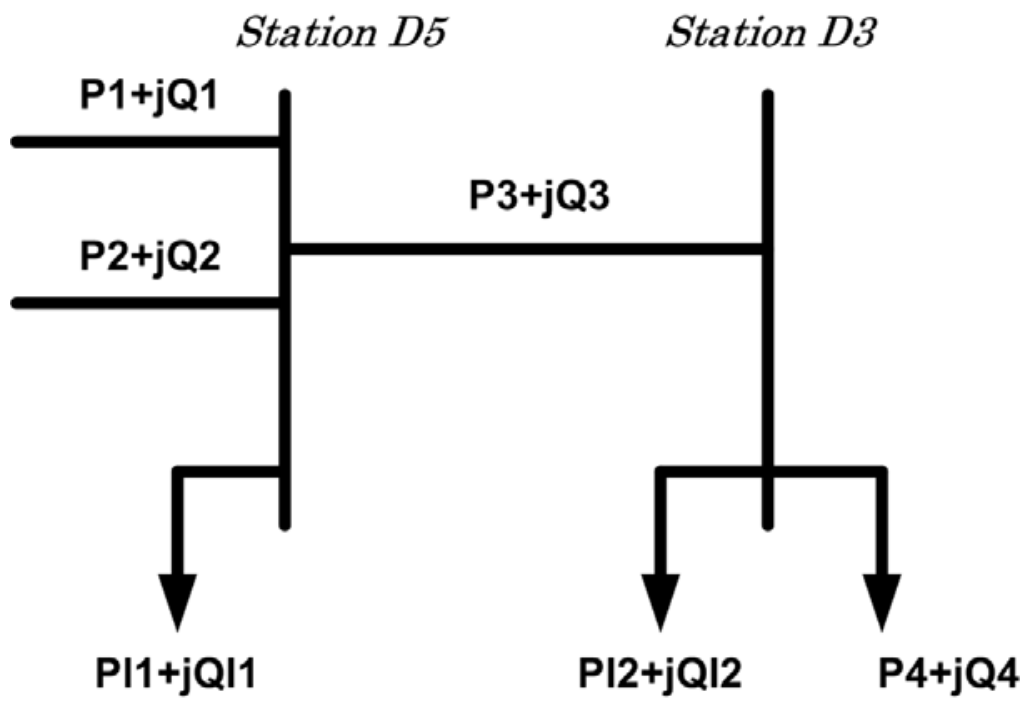

Figure 4: Modelling Cut-off Lines in Boundary Stations 
In Figure 2, it can be seen that Area-A and Area-C are connected through two tie lines. In addition, Area-A and Area-C are also connected via a high impedance path through AreaD. In the modelling method described above, the connection between Area-A and Area-C through Area-D will be lost. Therefore, unless utilities use a larger buffer zone to account for all the external impact this method will produce errors in the security assessment.

\subsubsection{Use of Unreduced Power System Model for the External System}

Most of the off-line studies typically start their analysis with the unreduced power flow model. Utilities are usually grouped based on reliability control regions and tasked to develop a set of power flow models to represent the model for a particular period [59]. Members of the model building group submit the load forecast, expected generation dispatch order, expected export and import, and outages to a central location. The model building group then use this information to generate a network admittance matrix. They use the matrix and operational data to come up with the best power flow model for the given time period. Members of the model building group then use this as the base case for their off-line studies. One of the ways to represent the internal and external system in the real-time security assessment is to use the same full system used in the off-line studies for the real-time studies. 
The objective of the real-time assessment is to provide an accurate analysis of the system based on the actual real-time conditions. The accuracy of the real-time security assessment greatly depends on the accuracy of the power flow model used since the realtime security assessment starts with this real-time power flow model. As mentioned above, network admittance can change due to planned or unplanned outages. The network admittance matrix can be reconfigured if the utility has access to the network status of the elements in real-time. Real-time operational data, which include active power output of the generators and load demand data, are required to generate the remaining part of the equations.

In order for a utility to perform a real-time analysis using the full models two conditions must be satisfied. First, it is necessary to develop the network model of the system. The model should show the network connections with the correct impedance of each link, location of generation and loads, etc. Second, the network model should be updated with real-time information. Modelling an entire area with the correct parameters in an EMS is time consuming and requires lots of resources. Therefore, once modelled, it is very important to allocate resources and create a process to keep the model up to date with system enhancements and changes. This is very challenging as there is no requirement to share the system enhancement of a particular area with other areas. It is even more challenging to update the operational data in the power flow model in real-time. The full model can not only go beyond a utility's own boundary, sometimes it can even go beyond the boundary of the reliability coordinator. This is illustrated with Manitoba Hydro's model. Manitoba Hydro is a member of the MRO (Midwest Reliability Organi- 
sation) model building group and participates in the regional model building process. In return, Manitoba Hydro receives the off-line model of the entire eastern interconnection. Manitoba Hydro uses this power system model in off-line studies. Figure 5 shows the North American interconnection regions. The MISO area (who is the Reliability Coordinator for Manitoba Hydro) is shown in the colour turquoise.

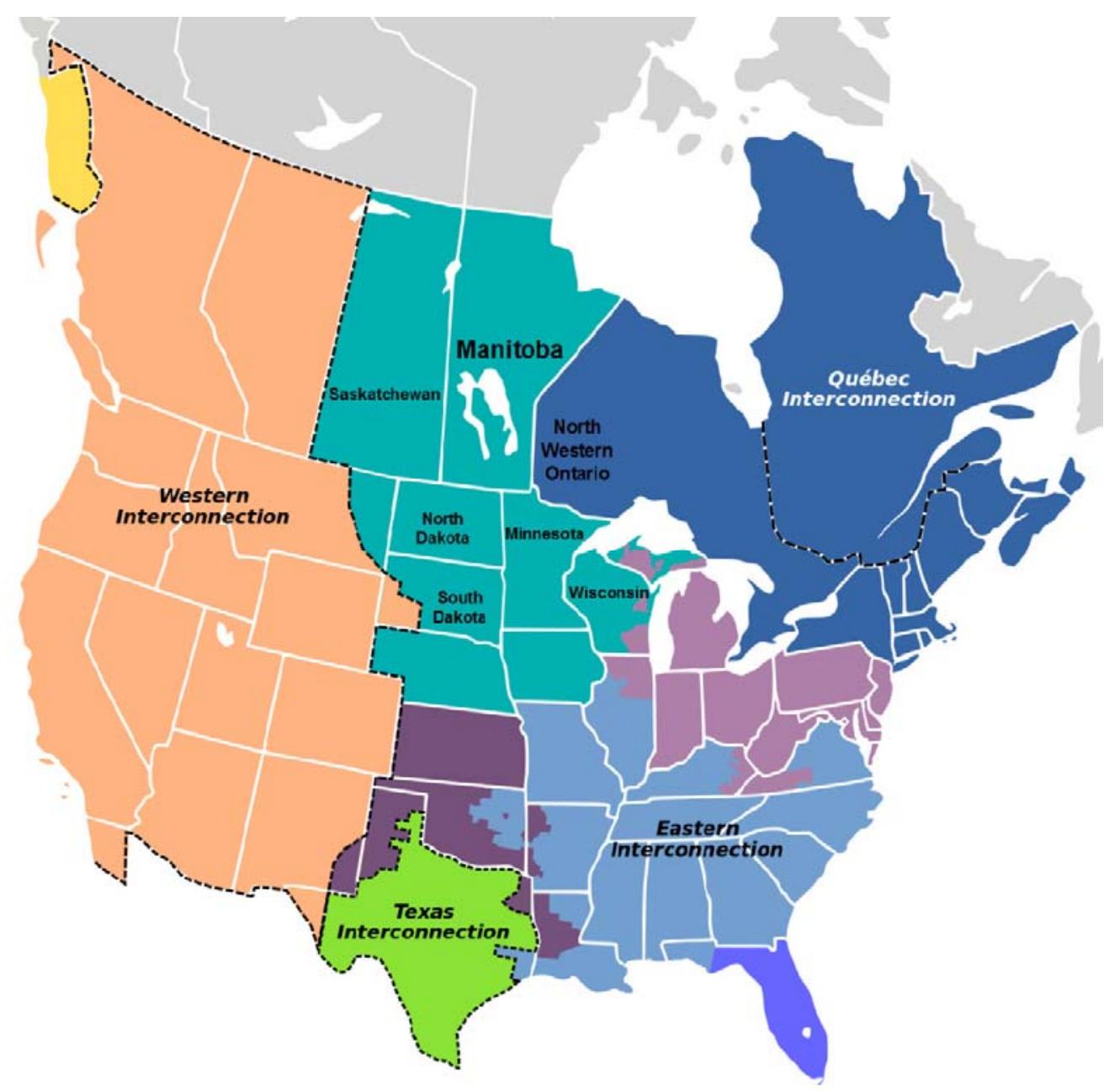

Figure 5: North American Interconnection Regions

Most control centres do not have real-time data of the entire external system. Therefore, construction of a real-time power flow model for the complete area is not a 
trivial task. Within utilitiy's own control area, there could be some small pockets of area where they do not have visibility. These areas are usually known as unobservable areas. Utilities use the data from the areas which are observable to estimate the parameters of the unobservable areas in the state estimator. Utilities use a similar concept to estimate the parameters of the external area of the power flow model by considering it as one large unobservable area. They use the available real-time data with various techniques to obtain the correct boundary condition and estimate the parameters for the external area [60$67]$.

Few utilities spend the time to match the real-time model area to the off-line model area. Instead they use the off-line studies to determine the cut-off region (where modelling beyond that will have only marginal impact to the accuracy of the analysis) and only model that area in the EMS.

\subsection{Use of an Equivalent Network to Represent the External System}

One of the most widely used methods in the industry to represent the external system is to use an equivalent circuit. This section describes the evolution of equivalent circuits over time and discusses some of the previous work carried out in the equivalent circuit's area. 


\subsubsection{Evolution of Equivalent Circuits over Time}

Prior to 1929 power systems studies engineers and researchers mainly used mathematical calculations to solve power flow equations. In 1929, the first ac network analyser, jointly designed and developed by Massachusetts Institute of Technology (MIT) and General Electric (GE), was installed in the Electrical Engineering Research Laboratory in the Massachusetts Institute of Technology at Cambridge, MA, USA [68]. Up until the 1960s, power system studies engineers and researchers around the world used network analyzers for operation and planning studies of the power system [68-72]. A network analyzer has a number of components such as generator units, line units, load units, and capacitors units to represent the power system under study. For example, a network analyzer installed in the United States Bureau of Reclamation had 12 generator units, 108 line units, 32 load units, and 42 capacitor units to model the power system [69]. Towards the late 1940s, the load and generation of power systems had increased and so too had the number of interconnections with neighbouring systems. As a result, power system engineers and researchers were forced to find a new method to represent the system with the available components in the network analyzer. This led to the start of research into equivalent circuits.

Ward's paper describes two practices used in the early 1940s to reduce the size of the network [73]. The first method is that the network that needs to be studied in detail is terminated at interconnecting points, and the adjacent system is represented by a load or generator attached at each of these terminals (referred to as stub loads and generators). 
Connections through the reduced system are omitted entirely and an estimated tie line flow, based on system readings or previous studies, is reproduced in the stub loads and generators at the interconnection terminals. However, with system changes, it is difficult to predict the value of the stub loads or generators at the boundary with sufficient accuracy, specifically for future conditions. In the second method, the system to be simplified is replaced by an equivalent circuit in which the identity of the tie-line terminals and generator busses are retained. Between these various busses, the line network is represented by an equivalent circuit. In 1949, Ward proposed a method to reduce the power system network while preserving the impact of the external system such that the reduced model could be modelled in the network analyzer [73].

Brown and Cloues noted that the industry use a different equivalent circuits for load flow studies and stability studies [74]. Therefore, it was necessary to modify the analyzer set up to perform stability studies after load flow studies and vice versa. Brown and Cloues proposed a new method to develop an equivalent circuit which could be used interchangeably for both load flow and stability studies [74].

In 1946, Jennings and George suggested that power system analysis could be performed by the accounting machines of the International Business Machine (IBM) type which was usually available to members of the light and power industry [75]. At this time, the power system industry slowly started to use digital computers for power system analysis [75-91]. With the use of digital computers, the power system industry no longer 
had to worry about the component limitations in the network analyzer and the need for equivalent circuits was diminished.

By the late 1960s, power systems had developed in size and complexity which demanded large numbers of power flow models to be solved for proper security assessments. Therefore the use of network analyzers even with equivalent circuits was not possible. The power industry found that in applications involving security questions and local control of an area for a large interconnected system it was often not economically practical to analyze the entire system due to the following reasons [92].

- Costly repetition of data processing and associated computation, since significant questions concerning system security would only appear in the proximity of a disturbance

- Excessive capital costs associated with computing facilities capable of processing large power networks

- Meter-placement costs and, at times, the practical difficulties associated with data gathering from remotely located or not wholly-owned parts of the interconnected system

- Reliability problems associated with handling the data for the entire network

At the same time, the power industry had shown the desire to use mini computers for power system analysis, which restored the need for equivalent circuits. Duran et al. identified the operational objective of the equivalent circuit as its ability to simulate the 
system conditions within the area of interest while retaining the accuracy of the results within acceptable operational limits when:

- Local disturbances such as equipment outages are present in the area

- Changes in operating conditions such as load and generation levels occur throughout the system [92].

They also identified the following difficulties in meeting the above operational requirements when using equivalent circuits:

- An equivalent network model is never exactly interchangeable with the original network from which it is derived. The best that it can do is to reproduce exactly a single set of operating conditions, the knowledge of which is generally assumed in the derivation of the equivalent. Approximate results are then obtained for moderately different operating conditions.

- Local disturbances in the area affect the neighbouring systems. Occasionally they may cause some components in the neighbouring system to reach operating limitations such as thermal or stability constraints. In the equivalent representations, however, the individuality of the neighbouring system components is lost so that it is no longer possible to detect occurrences of such events.

- Changes in operating conditions in the neighbouring systems affect, in general, the area of interest. In the equivalent representation, the effect of the variables at neighbouring system buses is aggregated at the boundary buses of the area such that the effect of changes in these variables may become difficult to trace. 
Due to the constraints listed above, Duran et al. shifted and broadened the approach of the equivalent circuit problem from the classical question of how to obtain the equivalent network. The new approach instead addressed the questions of how to choose the buses to be retained in the equivalent and how to use the equivalent for a wide set of operating conditions. They argued that it is necessary to retain the sub-system area called the buffer system between the internal area and external area as shown in Figure 1 for an accurate analysis. It is necessary to retain the complete model of the buffer system due to following reasons.

- The accuracy of the equivalent may be very sensitive to whether a bus is eliminated or not. For example, if the bus is electrically close to the study area.

- Operational limitations on neighbouring system components such as bus voltage levels or line current ratings may be violated due to area disturbances. Such conditions may prevail, for example, if power is rerouted through the neighbouring system due to area outages.

- Line power flows and voltages in the area of interest may be very sensitive to the current neighbouring system operating conditions. Generation and voltage levels in neighbouring buses are examples of such conditions.

By about the 1970s, the power system industry had access to more powerful third generation computers providing the opportunity to exercise greater control over the scheduling of power system facilities. More importantly, these advanced computers made it possible to realistically consider the application of control computers for the reliability aspect of system operation. An important consideration in the use of on-line computers 
for reliability applications was the development and testing of analytical techniques for monitoring the present status of the power system by developing a real-time power flow solution based on system measurements [62, 93-95]. The real-time power flow model derivation process used a state estimator to address any errors associated with measurements and missing data.

Power system operators faced a new challenge when it came to creating a realtime power flow model with the external system for the following reasons [62],

- It was not possible to obtain real-time operating data of the external area.

- Power utilities only modelled the major transmission elements, ignoring the majority of the sub-transmission network.

- Knowledge of forced or scheduled line outages of the external system was not always available

These factors forced the power industry to look into creating equivalent circuits to use with the state estimators $[62,63]$.

\subsection{Main Methods Used in Developing Equiva- lent Circuits}

A comprehensive review of the major methods used in the industry can be found in the literature [96-100]. Among the methods available, the most widely used methods in the industry are the Ward method, Residual Equivalent Independent (REI) method and some 
variation of the above two methods. This section provides an introduction to the Ward and REI methods, and discusses some other recent work on equivalent circuit development.

\subsubsection{Ward Method}

Ward proposed two methods called the Ward Injection method and the Ward Admittance method to develop equivalent circuits [73]. Both of these methods start with a solved power flow model containing external buses and boundary buses. Creation of an equivalent circuit involves reducing the admittance matrix of the external buses and the boundary buses using Gaussian elimination. The only difference between these methods is that in the Ward Admittance method, all bus powers in the networks are converted into shunt admittances before network reduction. As a result this converts the equivalent into a passive network. It has been reported that between these two methods, the injection method produces more accurate results than the admittance method [96].

When an external circuit contains mostly PQ buses, equivalent circuits produce better results than when the external circuit contains mostly PV buses [96, 98]. It has also been found that when contingency analysis was carried out using the Ward type equivalent circuit, it generally produced reasonably good results for the active power output. However, it generally produced poor results for the reactive power output $[96,98]$. 


\subsubsection{Ward PV Method}

As mentioned above, the Ward equivalent calculation could not produce the same level of accuracy when the reduced external network contained PV buses. Therefore, in the Ward PV equivalent method, the Ward reduction is only applied to the external PQ buses. The resultant equivalent has the Ward equivalent for the PQ buses while retaining the external PV buses. It is reported that the Ward PV equivalent produces good results for the contingency analysis [97]. However, this method requires keeping large numbers of external buses in the model. It is reported that acceptable accuracy can be achieved by only retaining small numbers of PV buses that are capable of producing large amounts of reactive power when needed [114]. However, this required development of criteria which specify how to select the PV buses to be retained under the different operating conditions.

\subsubsection{Extended Ward Method}

It is known that the Ward equivalent circuit produces reasonably accurate results for active power but less accurate results for reactive power. On the other hand, the Ward PV equivalent produces reasonably accurate results for both active power and reactive power. The objective of the Extended Ward equivalent is to combine the simplicity of the Ward equivalent with the response of the Ward PV equivalent [98]. 
Extended ward equivalent can be obtained from the Ward equivalent circuit by performing the following steps:

- Designate each boundary bus as a either PQ bus or PV bus.

- At each designated fictitious PV bus boundary, add a generator behind a fictitious impedance with power output of $0 \mathrm{MW}$ and voltage control to be the same as the boundary bus voltage.

The new generation buses added to the boundary do not contribute to the active power of the system or reactive power in the base case. However, whenever the study system conditions change, these boundary generation buses contribute by supplying or absorbing reactive power. It has been reported that the Extended Ward equivalent provides accurate results for contingency evaluation [97]. This method is praised for combining simplicity and good reactive power responses. One of the other advantages of this method is that Ward equivalent can be easily extended into the Extended Ward equivalent.

There is also another method called the "Simplified Extended Ward equivalent" circuit. In this method, the resistance of the external circuit was ignored and therefore the external equivalent circuit only contains reactance. More details on Ward type equivalent methods and how the power industry uses these equivalent circuits in various studies can be found in the literature [101-107]. 


\subsubsection{REI Method}

The Radial Equivalent Independent or REI method was first published by Paul Dimo [108] and bought to the attention of power engineers by Tinney and Powell [109]. The objective of the REI method is to aggregate the power injection of groups of buses into a fictitious REI bus. Therefore, each designated group is replaced by a single node. The bus grouping can be performed based on generation and load conformity, geographical area, electrical distance, etc.

For example: A group of buses to be eliminated have been identified and these buses connect to the set of boundary buses as shown in the Figure 6. The first step of creating a REI equivalent is to remove all the bus injections from the designated sources and add them into the fictitious node $\mathrm{R}$ as shown in Figure 7 . Therefore, the power injection at the REI bus, SR, is the summation of all the injection in the designated zone. Next connect the REI node, $\mathrm{R}$, to all the buses in the designated group through a fictitious node $\mathrm{G}$ as shown in the Figure 7. The impedance between node $\mathrm{G}$ and each bus, and the impedance between node $\mathrm{G}$ and node $\mathrm{R}$ is selected such that injection into bus $\mathrm{k}$ is equal to the impedance in the original unreduced power flow model. Finally, Gaussian elimination is used to eliminate all the buses in the designated area and the star point $\mathrm{G}$ to produce the final equivalent circuit as shown in the Figure 8. This process can be repeated to reduce all the identified groups into single REI nodes. 


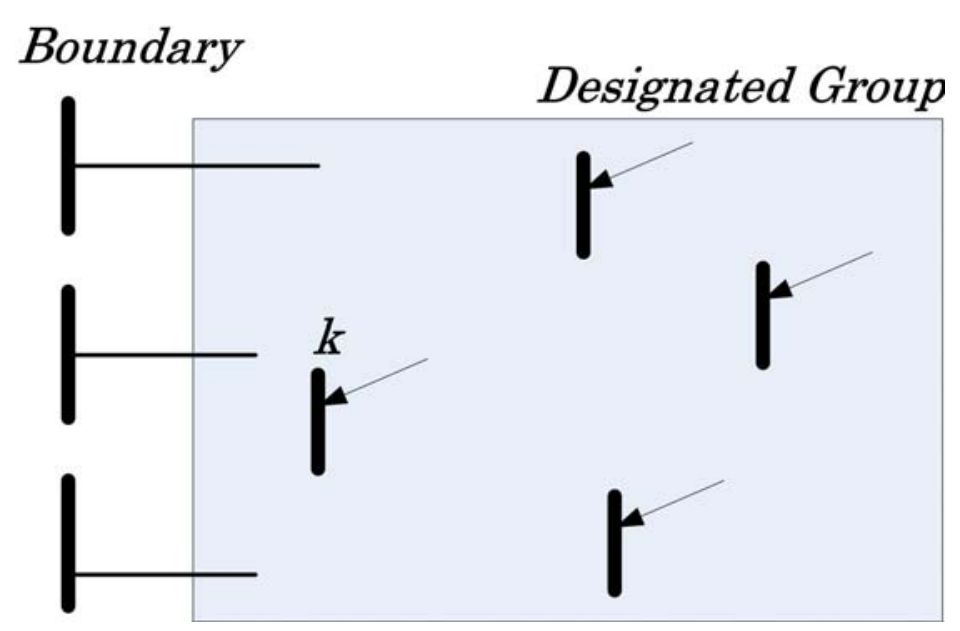

Figure 6: Step1-Identify the Group of Buses Connected to Boundary

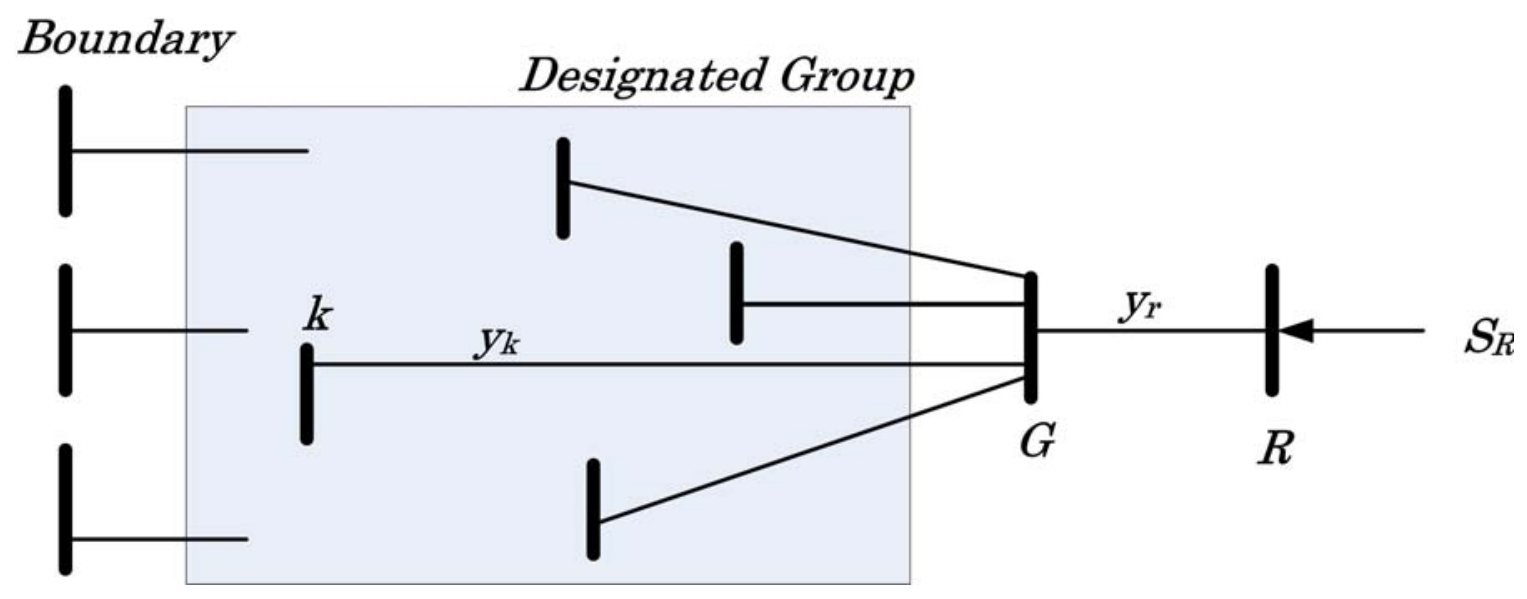

Figure 7: Step2-Adding Bus Injection in the Group to a Fictitious Node 


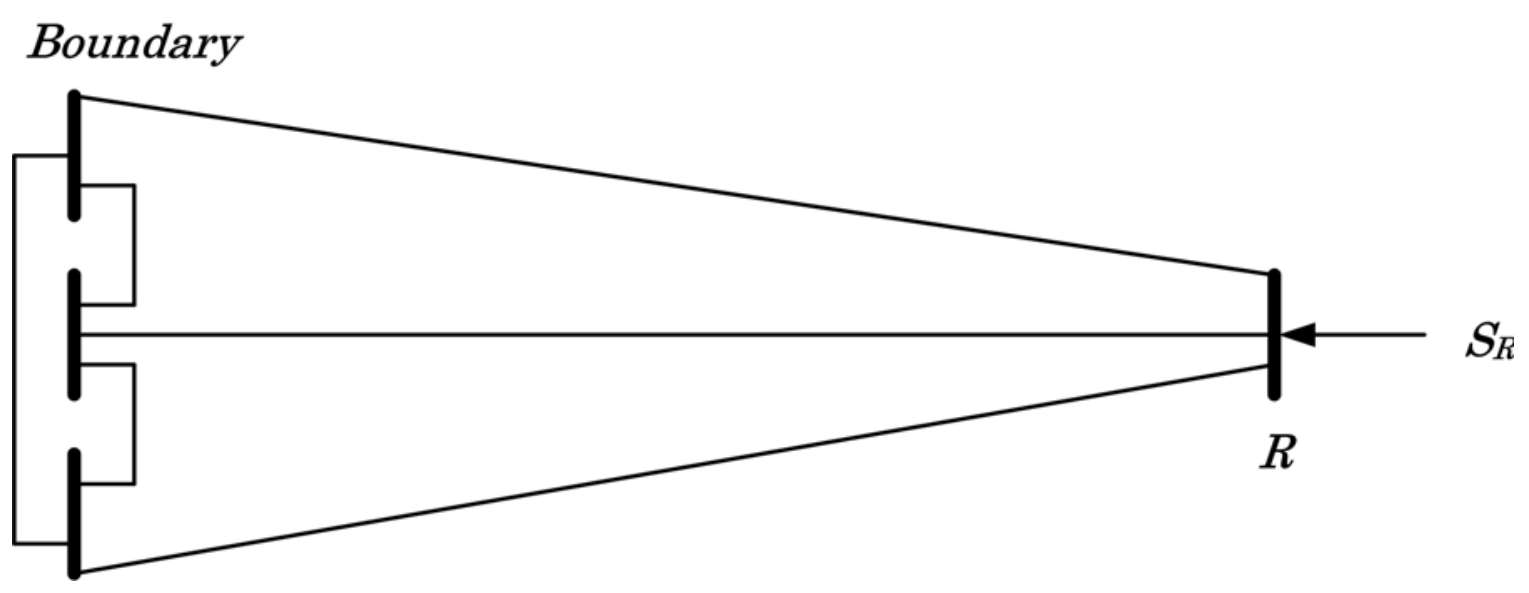

Figure 8: Step3-Use Gaussian Elimination to Remove all the Buses in the Designated Area

The REI method is derived from the solved power flow model. Impedances connecting the star node to other buses are derived to provide exact power injections into the buses in the designated area. Therefore, the REI method produces exact results for the same condition in which the power flow model was derived. However, it cannot be guaranteed that it will produce accurate results for other conditions $[96,98]$. Wu et al. carried out work to determine the necessary conditions for the REI equivalent to be accurate in other conditions [115]. The conditions they determined are only the necessary condition to produce accurate results, but they are not sufficient to produce accurate results.

The accuracy of the REI circuit for contingency analysis or conditions other than the original design also depends on the groupings of buses to be included into a REI node [98]. Various studies have been carried out on selecting the groups of buses to be reduced as REI nodes $[112,114]$. Since grouping depends on the state of the power system, groupings should be changed as conditions change. Alternatively, the number of groups 
can also be increased to improve accuracy. It is estimated that the number of groupings for a larger power system can range anywhere from 10 to 100 [109].

It has been reported that the REI equivalent produces poor results with respect to the active power and reactive power response $[96,98]$. It has been suggested that grouping generators into one group, and loads into another group, or not reducing some of the important buses can increase the accuracy [110].

\subsubsection{Adaptive Reduction Method}

Tinney and Bright proposed that network reduction can be performed automatically using computers [116]. This method is based on the network reduction method proposed by Tinney in an early publication [117]. The authors claimed this method could be used to compute equivalent circuits for any type of power flow model based on the matrix reduction. The authors used the method to develop an equivalent circuit using the Extended Ward equivalent. Some of the advantages of the Adaptive Reduction methods include [116]:

- Calculation of equivalent circuit is fast and can be automated.

- A customised equivalent circuit can be computed for each power flow problem and the equivalent can be adjusted to provide sufficient buffer zone.

- Calculated equivalents are always extremely sparse and there are no approximations involved. 
- The sparse matrix/vector operations used in the Adaptive Reduction method is used by other power system applications.

\subsubsection{External Network for Optimal Power Flow Applications}

Over time, power utilities have changed from the traditional monopoly supplier, where a single organization was responsible for the generation, transmission, and distribution of electricity into deregulated electricity market [118-128]. As the electricity market evolved it exposed new concepts such as Optimal Power Flow (OPF), marginal pricing, binding constraints, etc. The electricity market requires utilities to solve the optimization problem in order to find the cheapest set of generation to serve customer loads while respecting security constraints. This requires utilities to solve an optimal power flow periodically based on the current network structure to find out the optimum generation set that can meet the electricity demand subject to system and generator constraints. Due to the same reasons explained earlier in the text, this power flow model should not only represent the internal area, but it should also represent the external area. This creates a need for the equivalent circuit.

It has been found that the power flow based equivalencing methods may not produce acceptable results in the optimal power flow condition even under the base case solution [129]. The errors are mostly associated with the modelling of losses in the equivalent model and inability to monitor or enforce inequality constraints in the equivalent area [130]. If the losses of the equivalent are too low, an OPF will route more power through the external area, and if the losses of the equivalent are too high, the results will be oppo- 
site. At the same time, if the equivalent circuit is unconstrained, an OPF will avoid the binding constraints in the internal area by routing power through the external area.

To avoid these shortcomings Hao et al. proposed a new method to develop an equivalent circuit to use in the OPF applications developed in the Pacific Gas and Electric Company in California [130]. This method involves the following steps:

- Buses of the full model are divided as internal, boundary and external based on operators experience and the preliminary analysis.

- Reduction process is used to eliminate the external buses and to modify the boundary buses. Buses in the internal area are kept in the reduced model without any changes.

This method is similar to the Extended Ward method. However, it has the following two distinctions:

- Extended Ward method uses the admittance matrix to perform network reduction, where as the proposed method uses the Jacobian matrix in the network reduction.

- Proposed method respects the gradient optimal condition as well as the power flow balance equation when performing boundary matching.

The authors claimed that this method outperformed the Extended Ward equivalent and hence could be used not just in OPF exercises but also in security control as well. The literature review provides a few more examples where equivalent circuits are used in various optimization works [131-133]. 


\subsubsection{PTDF Based Power System Equivalents}

Efficient operation of the deregulated electricity sector requires tools to analyze behaviour of the electricity market. These tools are typically computationally demanding particularly when performing long term market simulations [134]. For example, it was reported that performing a dc OPF solution for the Western Electricity Coordinating Council (WECC) area (which contains 15,000 buses and 18,000 branches) took 15 minutes using MATPOWER [135]. Therefore, it is not possible to perform various long term market assessments using the full power system model. As a result, market operators were forced to use equivalent circuits in their analysis.

The NERC glossary of terms define the Power Transfer Distribution Factors (PTDFs) as "a measure of the responsiveness or change in electrical loadings on transmission system facilities due to a change in electric power transfer from one area to another, expressed in percent (up to $100 \%$ ) of the change in power transfer" [136]. PTDFs have proved to be a useful technique for congestion management in a deregulated market [137-140]. Therefore, PTDFs are widely used in various tools in the deregulated electricity market. PTDFs can be calculated using either ac power flow models or dc power flow models.

Tools associated with a large market require equivalents to retain the geographical scope of the entire network, retain most or all of the load and generation, and correctly model PTDF associated with desired market participants. However, the traditional 
method of calculating equivalent circuits does not provide this capability. Therefore, there was a need to develop a new method to develop equivalent circuits for these kinds of applications.

When the PTDF of a particular bus is calculated with respect to a system swing bus, they are called Injection Shift Factor (ISF). When the power exchange is between two regions rather than two buses, these ISFs are called regional ISF. Cheng and Overbye developed a method in which they used the matrix of the calculated regional ISF to come up with the equivalent impedance in the equivalent circuit [134]. They used this method to reduce a 12,925 bus systems into a 25 bus system. One of the drawbacks of this method is that it requires updating the ISF matrix and one of the other intermediate matrices (called F matrix) to calculate the equivalent impedance matrix when the operating condition changes [141]. Therefore, this method cannot be used for various planning studies where system configuration could change. Oh proposed a method where the equivalent circuit developed does not depend on the operating point in order to overcome this problem [86]. His method involves the following steps:

- Define the areas, so that all the buses in the area have the same properties.

- Ignore all the lines connecting two buses that belong to the same area.

- Lines connecting two buses that belong to the same area are aggregated.

- PTDF of the reduced network is computed.

- Reactance values for the reduced network are computed from the PTDF (obtained in the previous step) and the node branch incident matrix of the reduced network. 
He used his method, Ward method and original PTDF method proposed by Cheng and Overbye to reduce the WECC system to a 180 bus system and compared the results. For them it took $8 \mathrm{hrs}$ to develop the ISF matrix necessary for to create equivalent circuit for Cheng and Overbye method. However, Oh developed entire equivalent circuit using his method using 8 hours. When compared with the two PTDF methods both of them produce the same level of accuracy for the condition in which it was developed. However, Oh's method outperformed the original PTDF method, when tested for a different condition.

Both of PTDF methods used a dc power flow. Therefore, these methods do not address issues such as voltage and reactive power. The methods may also perform poorly in cases where the de power flow model is not a good approximation to the ac power flow model.

Most of the equivalent circuit creation method for OPF require grouping of generators. However, there are no clear guidelines as to how the grouping of generators should be carried out. Yet it is very important to aggregate buses with similar behaviour into the same group. On the other hand, congestion plays a critical role in the OPF and various planning studies. Congestion arises when the flow is equal to the line limit. Therefore, it is important to have the appropriate line limits not only for the internal lines but also for the lines in the equivalent network. Oh produced another paper to address these two issues [142]. 


\subsubsection{Equivalent Network for Policy Analysis}

Various studies related to public policies require analysing the impact of the policy on the various sectors in details. Analysing generation and investment policy options in a transmission constrained electric market is one such example [143-146]. These kinds of studies require developing an equivalent system of the entire region. The concept of traditional equivalent circuits, shown in Figure 1 is no longer suitable for this kind of study. Instead it is necessary to develop an equivalent circuit of the entire system where the internal system is spread over the entire study region.

Shi at el. used the modified Ward equivalent to develop an equivalent circuit of the Eastern Interconnection, which has approximately 62000 buses, 8000 generators and 80000 branches, into a 273 bus equivalent to use in the system planning study associated with congestion and various green house emission policies [143]. The steps they followed to develop the equivalent circuit include:

- Select the buses to be retained in the equivalent circuit. For their studies they kept the buses in the area where they expected the congestion.

- Remove small islands in the Eastern interconnection and replace high voltage dc lines as two generators at both ends of the line.

- Use the Ward equivalencing procedure to reduce the network other than reduced buses.

- Use the Ward reduction again this time keeping all the buses that were retained in the previous step and all the generator buses. 
- Use the two equivalents derived in the previous two steps to move the generators located in the external area into the boundary. The generator's active power limit was set to the original value and the reactive power limit was ignored since the intention of the study was to use the OPF with de power flow.

- Use the inverse power flow solution to move the loads into the buses so that power flow on the reduced model is exactly the same as the full model.

\subsubsection{Reduced Network model for Fast Nodal Price Calculation}

One of the requirements of the electricity market is to market response to the change in the electricity prices. For this purpose, it is necessary to calculate the marginal price of the electricity for the various nodes. This requires utilities to calculate the marginal price in the near real-time for a large number of nodes and communicate the price to electricity customers in frequent intervals. In order to reduce the number of nodes that require marginal price calculations, Singh and Sirivastava [147] proposed to reduce the number of nodes in the network using the REI method [108, 109]. As previously discussed, the REI method of reducing a network includes selecting groups of buses and reducing them into a single REI node. To select the buses for a single node Singh and Sirivastava used the following method:

- All the nodes in a group must have relatively close marginal prices.

- The nodes must be geographically close to each other.

- For a change in system loading, the differential marginal prices at all nodes in the group must remain relatively close to each other. 
Some of the equivalent nodes in the reduced model had to be designated as PV nodes and others needed to be designated as PQ nodes. They used the following logic to categorize equivalent buses as PV and PQ types:

- Groups of nodes without a generator participating in the OPF were categorized as a PQ node.

- Groups of nodes with at least one generator participating in the OPF were categorized as PV nodes.

For a selected PV node, if only one generator participates in the OPF, they used the properties of the generator as properties of the equivalent unit. However, when a node had two or more generators participating in the OPF, they used the least square approximation to calculate the properties of the equivalent generator unit [148]. The method was tested using the IEEE 118 bus system [149].

\subsection{Summary}

Utilities use different ways to present the external area in the real-time security analysis. Three most common methods include ignoring external area, use equivalent circuit, use full model to represent the external area. Use of equivalent circuit is the most common method use in the industry. 
Developing equivalent circuit research goes to more than 50 years. This Chapter described the development of equivalent circuit research over the period and various methods presented by the previous researches. From the available methods, Ward method and REI method is very popular in the industry. Both of these methods use impedance reduction methods to reduce the external area impedance to develop equivalent circuit.

Unfortunately utilities do not have external impedance information in the real time. Therefore, utilities cannot produce the external circuit in real-time using existing methods. The Chapter 3 presents a new method to develop equivalent circuit based on the available boundary information. 


\section{Chapter 3}

\section{Development of an Equivalent Circuit}

\section{using Linear Regression Method}

This chapter presents a novel method to develop an equivalent circuit using available boundary information. Boundary matching technique that can be used to connect the equivalent circuit to the internal is also presented.

\subsection{Proposed Methodology}

Consider the part of the interconnected power system shown in the Figure 2 with areas A, $\mathrm{B}, \mathrm{C}$ and $\mathrm{D}$, and the tie lines which connect those areas. This figure is reproduced again as Figure 9 as easy reference. Area-A is the entity interested in carrying out real-time transfer analysis and its EMS model contains all the major electrical equipments in AreaA, parts of Area-B, Area-C, and Area-D as shown in the diagram. Station $\mathrm{C} 1$ is the 
boundary station in Area-C and included in the Area-A EMS model. Similarly stations D1, D2, D3 and D4 are the boundary stations in Area-D and included in the Area-A EMS model.

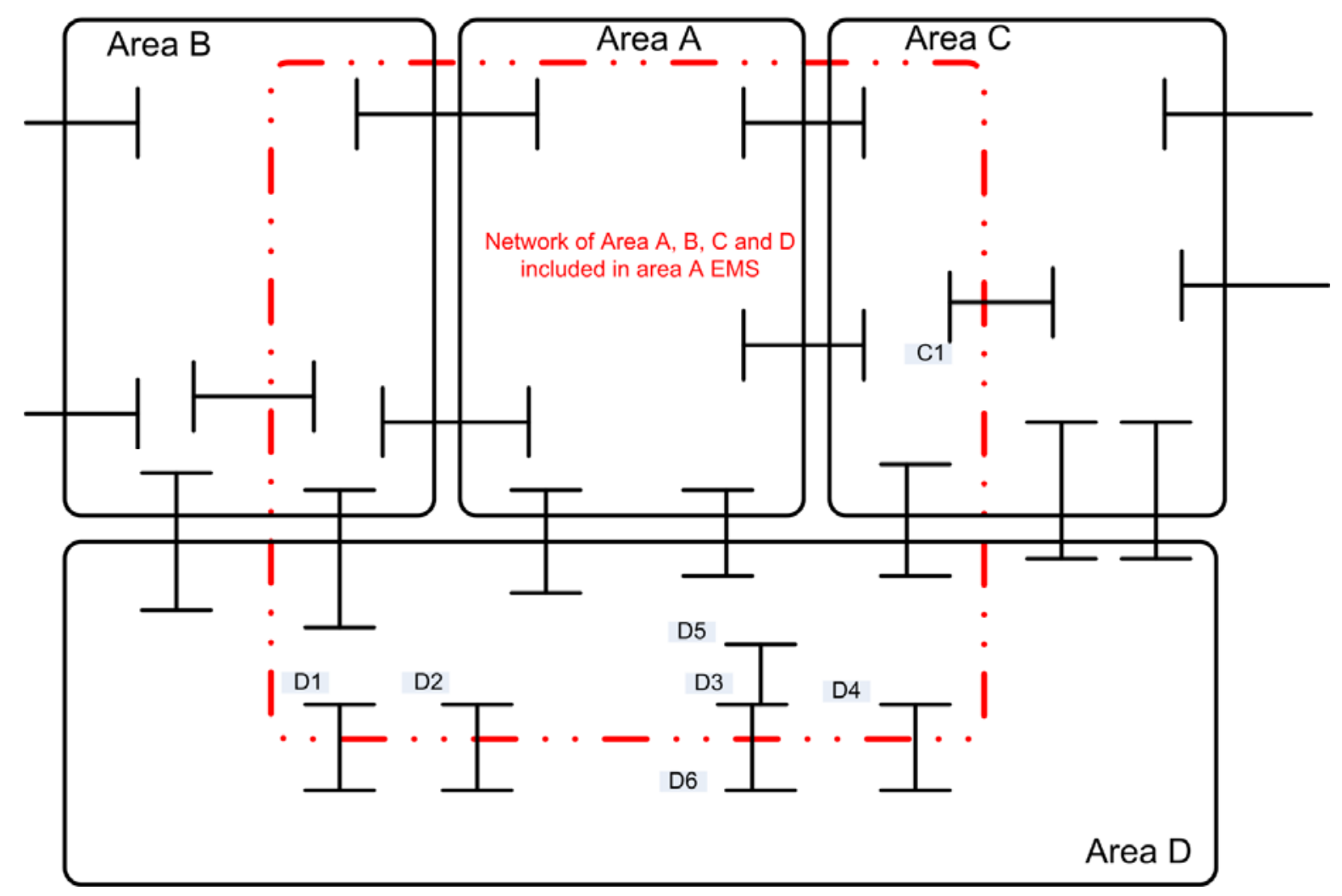

Figure 9: Part of the Interconnected System

Now consider the interaction between Area-A and Area-C. Area-A and Area-C are connected through two tie lines. In addition, Area-A and Area-C are also connected via a high impedance path across Area-D. Since Area-A's EMS model does not contain this information, it is necessary to develop an equivalent circuit to model these details. 
First, let's consider boundary stations D3 and C1. Assume that there exists a fictitious transmission line between station $\mathrm{D} 3$ and $\mathrm{C} 1$ and the admittance of this line is $\mathrm{Y}_{12}$. The objective of this exercise is to determine the admittance of this fictitious transmission line. The detailed representation of station $\mathrm{D} 3$ and $\mathrm{C} 1$ is shown in Figure 10, where $\mathrm{P}_{53}$ is the active power coming into station D3 from Station D5 and $\mathrm{V}_{1} \angle \theta_{1}$ and $\mathrm{V}_{2} \angle \theta_{2}$ are the voltage magnitudes and angles of station $\mathrm{D} 3$ and $\mathrm{C} 1$, respectively.

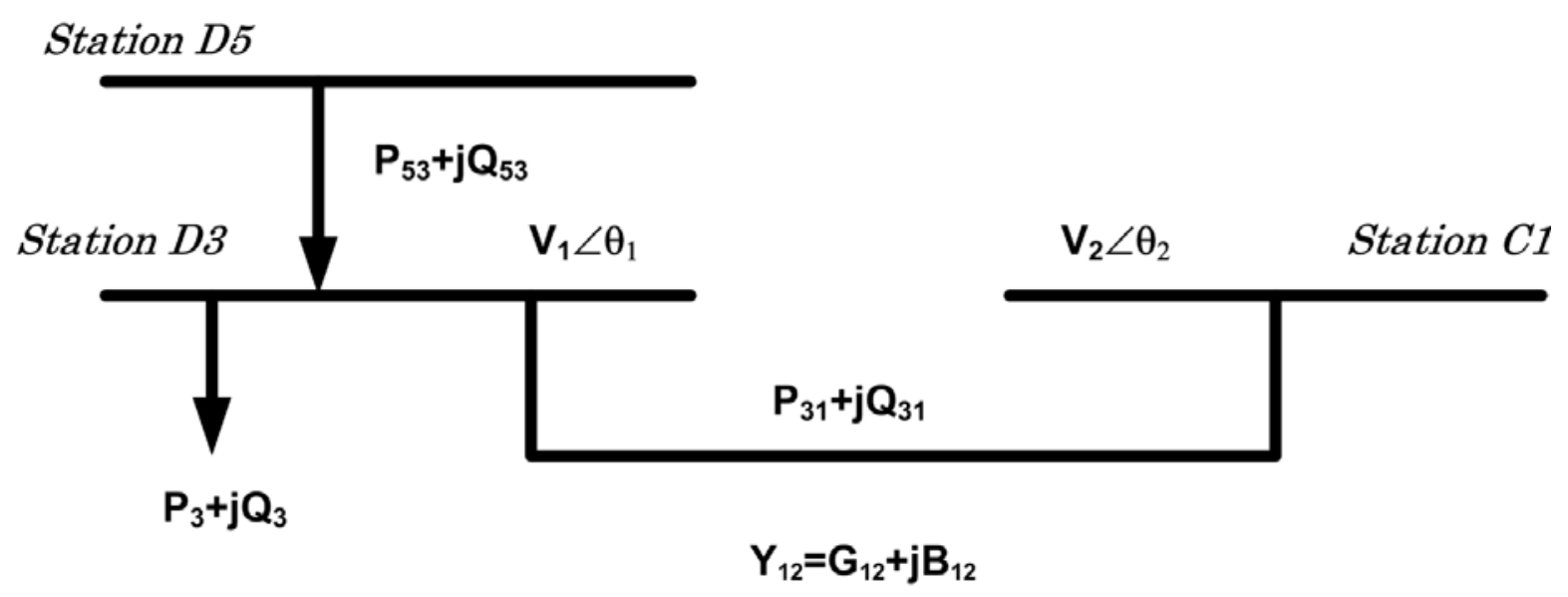

Figure 10: Estimating Equivalent Circuit Parameters

The active power flow into station D3 from station D5 $\left(\mathrm{P}_{53}\right)$ should be equal to the active power flow from station $\mathrm{D} 3$ to $\mathrm{C} 1\left(\mathrm{P}_{31}\right)$ and load $\mathrm{P}_{3}$. The load $\mathrm{P}_{3}$ represents the load at station D3 and the power from station D3 to rest of area D.

The following equation can be written for active power balance for station D3

$$
\mathrm{P}_{53}=\mathrm{P}_{3}+\mathrm{P}_{31}
$$


Power flow from station D3 to $\mathrm{C} 1$ can be written as:

$$
P_{31}=V_{1} V_{2}\left[G_{12} \cos \left(\theta_{1}-\theta_{2}\right)+B_{12} \sin \left(\theta_{1}-\theta_{2}\right)\right]
$$

Assuming that G12 is small compared to B12 for transmission network, the above equation can be re-written as:

$$
P_{53}=P_{3}+B_{12} V_{1} V_{2} \sin \left(\theta_{1}-\theta_{2}\right)
$$

Equation (5) represents a straight line. Therefore, if the power flow in the boundary stations can be plotted against voltage magnitude multiplied by phase angle difference $\left(V_{1} V_{2} \sin \left(\theta_{1}-\theta_{2}\right)\right)$ for different conditions, $B 12$ can be determined from the gradient of that line.

This approach can be used to determine the impedance between D1-C1, D2-C1 and D4-C1 as well. With these equivalent circuit impedances derived, the external equivalent circuit between Area D and C can be constructed.

\subsection{Developing Equivalent Circuit for Extended}

\section{0 bus system}

The IEEE three area 300 bus system [151] was used with a minor modification to demonstrate the equivalent circuit creation process and to validate the equivalent circuit parame- 
ters. In the three area test system, Area-1 is connected to Area- 2 through three $230 \mathrm{kV}$ tie lines (bus 131-7, bus 150-3 and bus 144-62) as shown in Figure 11. Area-1 is then connected to Area-3 via two $66 \mathrm{kV}$ lines (201-204 and 207 to 206), three $115 \mathrm{kV}$ lines (69211, 79-211 and 80-211), and two $345 \mathrm{kV}$ lines (81-194 and 81-195). The diagram only shows the tie lines connecting different areas for clarity. Drawings of the entire bus system can be found elsewhere $[152,153]$. In the three area 300 bus system, the only connection between Area-2 and Area-3 is across Area-1. In order to create another connection, a new area called Area-4 was added between Area- 2 and Area-3 as shown in Figure 12. 


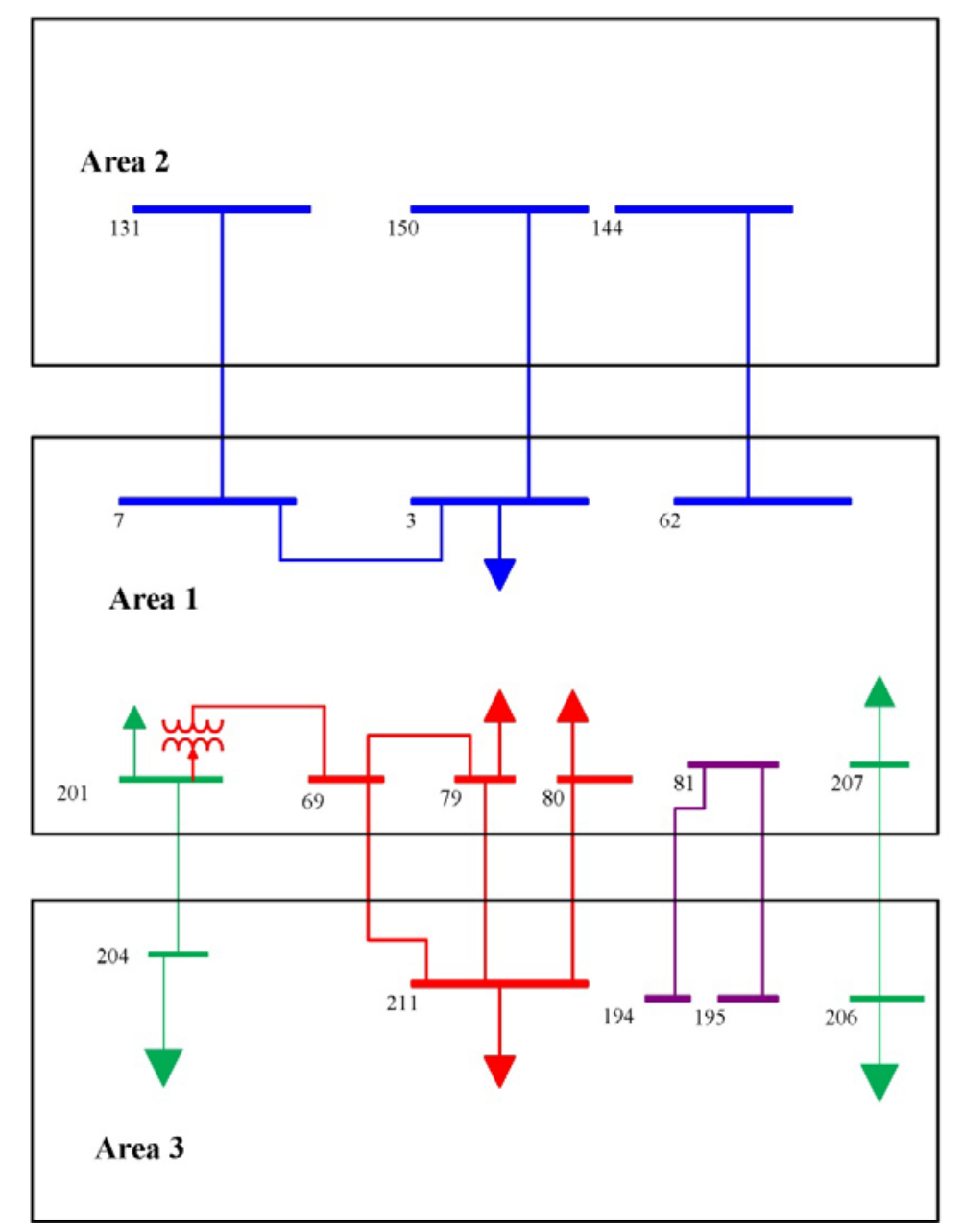

Figure 11: Three area 300 Bus System

There are four tie lines between Area-4 and Area-3. They include a $345 \mathrm{kV}$ tie line between bus 664 and 731, a $230 \mathrm{kV}$ tie line between bus 281 and 721, a $115 \mathrm{kV}$ tie line between bus 235 and 711 and a $66 \mathrm{kV}$ tie line between 250 and 761 . Area-4 is only connected to Area-2 through one $230 \mathrm{kV}$ tie line between bus 161 and 725 . 


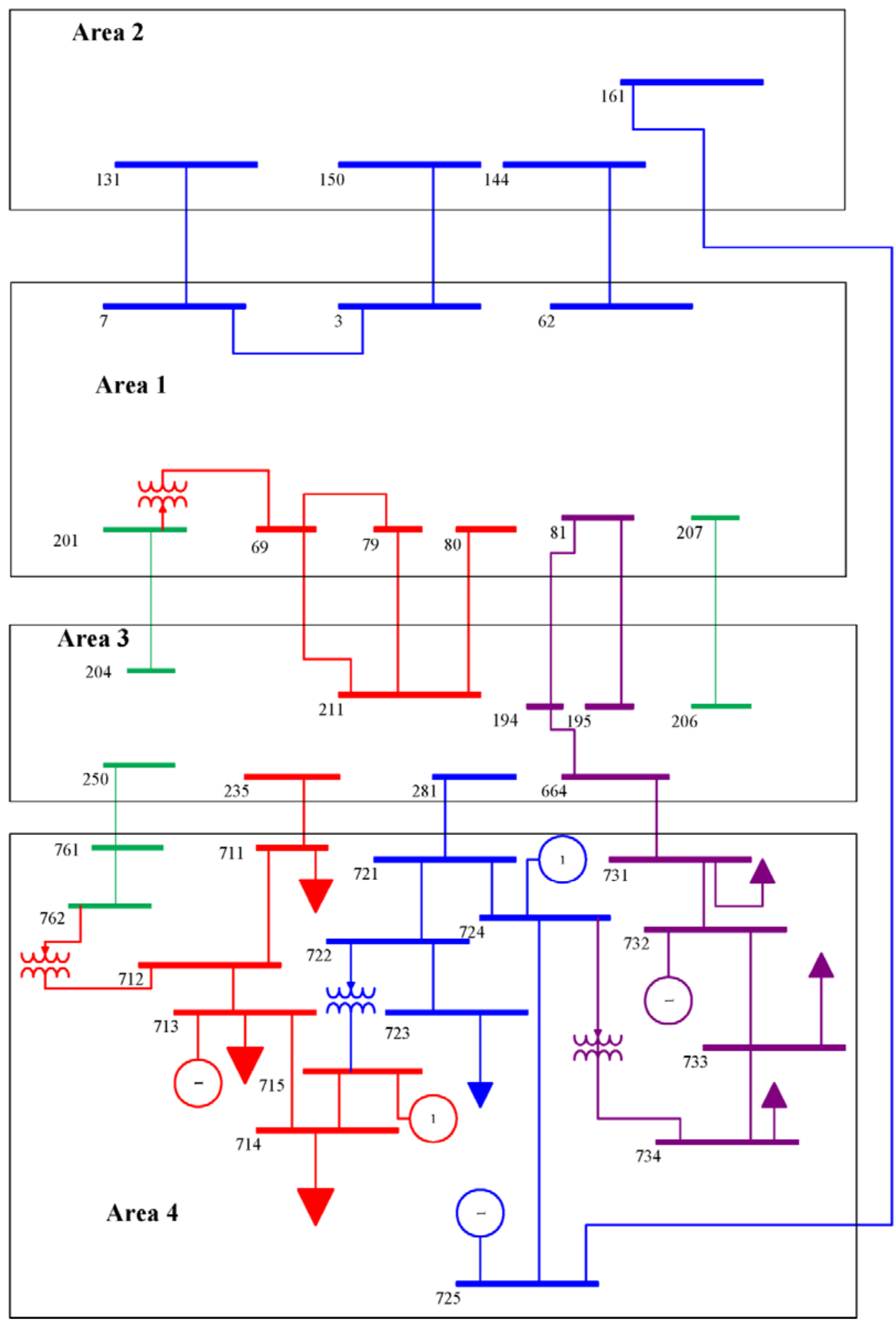

Figure 12: Three Area System with Added External Area 
Comparing Figure 12 with Figure 9, it can be seen that Area-1 in Figure 12 is equivalent to Area-A in Figure 9. Area-2 and Area-3 in Figure 12 are equivalent to the network of Area-C and Area-D, which are included in the Area-A EMS model. Similarly, Area-4 is equivalent to the network of Area-C and Area-D that are not included in the Area-A EMS model. The power system model shown in Figure 12 is the full system. But AreaA's EMS only has the power system model shown in Figure 11. The objective of this exercise is to create an equivalent circuit to represent the impact of external Area-4 to be used in the Area-1 EMS model.

In order to simulate a change in the operating state of the power system, a transfer scenario was created between Area-3 and Area- 2 in the modified power flow shown in Figure 4 by increasing generation in Area-3 and increasing load in Area-2 by steps of 20 MW until the generators in Area-3 reach their maximum capacity. When generation in Area-3 was increased with load in Area-2, the excess power in Area-3 had two paths to flow into Area-2. One path was across Area-1 and the other path was across Area-4. The amount of active power flow at each simulation step when the transfer level is increased by $20 \mathrm{MW}$ in each step is shown in Table 1 . The second column of the table shows the amount of power that flowed from Area-3 to Area-1. The third column shows the amount of power that flowed from Area-3 to Area-4. The remaining four columns show how the flow distributed across the four tie lines between Area -3 and Area-4. The last column shows the difference between the initial point and the final point. 
Table 1: Active Power Flow Across Various Interfaces and Paths

\begin{tabular}{|c|c|c|c|c|c|c|}
\hline Point No. & $\begin{array}{l}\text { From } \\
\text { Area-3 to } \\
\text { Area-1 }\end{array}$ & $\begin{array}{l}\text { From } \\
\text { Area-3 to } \\
\text { Area-4 }\end{array}$ & $\begin{array}{l}\text { From bus } \\
235 \text { to } 711 \\
(138 \mathrm{kV})\end{array}$ & $\begin{array}{l}\text { From bus } \\
250 \text { to } 761 \\
(66 \mathrm{kV})\end{array}$ & $\begin{array}{l}\text { From bus } \\
281 \text { to } 721 \\
(230 \mathrm{kV})\end{array}$ & $\begin{array}{l}\text { From bus } \\
664 \text { to } 731 \\
(345 \mathrm{kV})\end{array}$ \\
\hline 1 & -33.7 & -59.5 & -0.1 & -0.3 & -54.9 & -4.1 \\
\hline 2 & -13.5 & -55.5 & 1.2 & -0.4 & -52.4 & -3.9 \\
\hline 3 & 6.7 & -51.6 & 2.5 & -0.5 & -50.0 & -3.7 \\
\hline 4 & 26.9 & -47.7 & 3.9 & -0.6 & -47.6 & $\begin{array}{l}-3.4 \\
\end{array}$ \\
\hline 5 & 47.0 & -43.8 & 5.2 & -0.7 & -45.1 & -3.1 \\
\hline 6 & 67.0 & -39.8 & 6.5 & -0.7 & -42.7 & -2.9 \\
\hline 7 & 87.0 & -35.9 & 7.8 & -0.8 & -40.3 & -2.6 \\
\hline 8 & 107.0 & -32.0 & 9.1 & -0.9 & -37.8 & -2.4 \\
\hline 9 & 126.9 & -28.1 & 10.4 & -0.9 & -35.4 & -2.1 \\
\hline 10 & 146.8 & -24.1 & 11.7 & -1.0 & -33.0 & -1.8 \\
\hline 11 & 166.6 & -20.2 & 13.0 & -1.1 & -30.6 & -1.5 \\
\hline 12 & 186.4 & -16.3 & 14.3 & -1.2 & -28.1 & -1.3 \\
\hline 13 & 206.2 & -12.4 & 15.6 & -1.2 & -25.7 & -1.0 \\
\hline 14 & 225.9 & -8.6 & 16.8 & -1.3 & -23.3 & -0.8 \\
\hline 15 & 245.6 & -4.7 & 18.1 & -1.4 & -20.9 & -0.5 \\
\hline 16 & 265.3 & -0.8 & 19.4 & -1.5 & -18.5 & -0.3 \\
\hline 17 & 284.9 & 3.1 & 20.7 & -1.5 & -16.1 & 0.0 \\
\hline 18 & 304.4 & 7.0 & 22.0 & -1.6 & -13.7 & 0.3 \\
\hline
\end{tabular}




\begin{tabular}{|c|c|c|c|c|c|c|}
\hline 19 & 323.8 & 10.9 & 23.3 & -1.7 & -11.3 & 0.6 \\
\hline 20 & 343.2 & 14.9 & 24.6 & -1.8 & -8.8 & 0.8 \\
\hline 21 & 362.5 & 18.8 & 25.9 & -1.8 & -6.3 & 1.1 \\
\hline 22 & 381.8 & 22.8 & 27.3 & -1.9 & -3.9 & 1.3 \\
\hline 23 & 401.0 & 26.8 & 28.6 & -2.0 & -1.4 & 1.6 \\
\hline 24 & 420.2 & 30.8 & 29.9 & -2.0 & 1.1 & 1.8 \\
\hline 25 & 439.4 & 34.7 & 31.3 & -2.1 & 3.5 & 2.0 \\
\hline 26 & 458.5 & 38.6 & 32.6 & -2.2 & 6.0 & 2.2 \\
\hline 27 & 477.6 & 42.5 & 33.9 & -2.2 & 8.4 & 2.5 \\
\hline 28 & 496.6 & 46.5 & 35.2 & -2.3 & 10.9 & 2.7 \\
\hline 29 & 515.6 & 50.4 & 36.5 & -2.4 & 13.3 & 3.0 \\
\hline 30 & 534.5 & 54.3 & 37.8 & -2.5 & 15.8 & 3.2 \\
\hline 31 & 553.4 & 58.2 & 39.1 & -2.5 & 18.2 & 3.4 \\
\hline 32 & 572.2 & 62.2 & 40.5 & -2.6 & 20.6 & 3.7 \\
\hline 33 & 591.0 & 66.1 & 41.8 & -2.7 & 23.1 & 3.9 \\
\hline 34 & 609.7 & 70.0 & 43.1 & -2.8 & 25.5 & 4.2 \\
\hline 35 & 628.4 & 73.9 & 44.4 & -2.8 & 27.9 & 4.4 \\
\hline 36 & 647.0 & 77.8 & 45.7 & -2.9 & 30.4 & 4.7 \\
\hline Difference & 680.7 & 137.3 & 45.8 & -2.6 & 85.3 & 8.8 \\
\hline
\end{tabular}

By looking at column two and three, it can be seen that a total of $818 \mathrm{MW}$ of power transferred from Area-3 to Area-2. Of that, $680.7 \mathrm{MW}$ or $83.2 \%$ of power flowed 
across Area-1 into Area-2 and only $137.3 \mathrm{MW}$ or $16.8 \%$ of power travelled across Area-4 into Area-2. Further investigation into the power in Area-4 showed that power flow changes across $66 \mathrm{kV}$ boundary buses 250 to 761 were very small. Therefore, for the purpose of creating an equivalent circuit between Area-3 and Area-2 to represent Area-4, the connection across $66 \mathrm{kV}$ was ignored. The base power flow from Line 250 to 761 was modelled as a load in the equivalent circuit.

In order to calculate the admittances between the other three boundary buses in Area-3 and Area-2, power transferred from each of the boundary buses in Area-3 at different transfer conditions is plotted against $V_{1} V_{2} \sin \left(\theta_{1}-\theta_{2}\right)$. These plots are shown in Figure 13 to Figure 15. The gradients of those plots were used to calculate the equivalent impedance between each boundary bus in Area-3 and the boundary bus in Area-2. Table 2 shows the calculated equivalent impedance for each path with the correlation coefficient. Figure 16 shows the three area 300 bus system with the equivalent network. In this model, the entire Area-4 is replaced by three lines (2350-1610, 6640-1610 and 281-1610) from Area-3 to Area-1. Two zero impedance transformers between 235-2350 and 664 to 6640 are added to account for different voltage levels and a zero impedance branch was added between bus 1610 to 161 . 


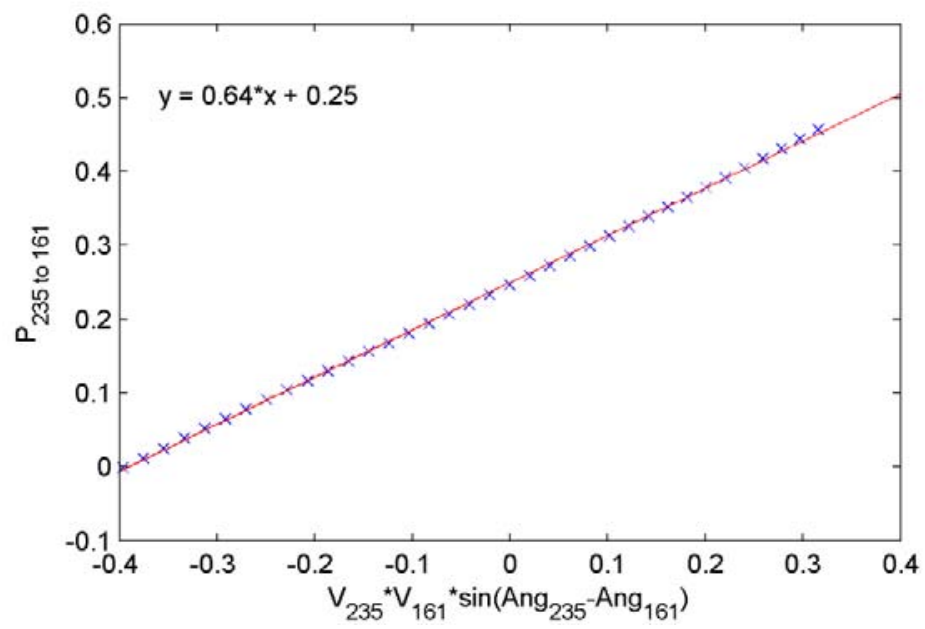

Figure 13: Plot of Power Flow into Boundary Station with Voltages and Angle Difference Multiplication for 138 kV Boundary Station

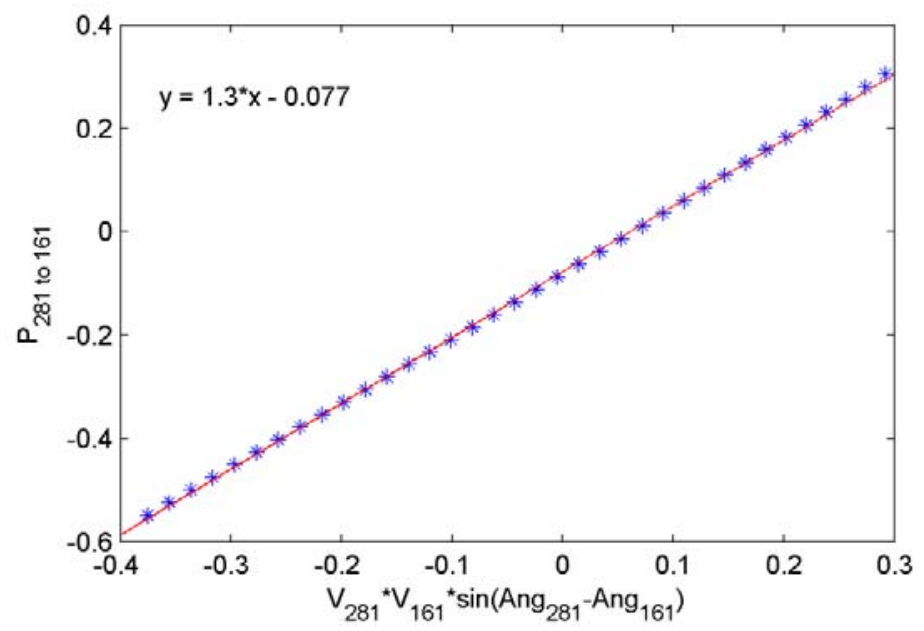

Figure 14: Plot of Power Flow into Boundary Station with Voltages and Angle Difference Multiplication for 230 kV Boundary Station 


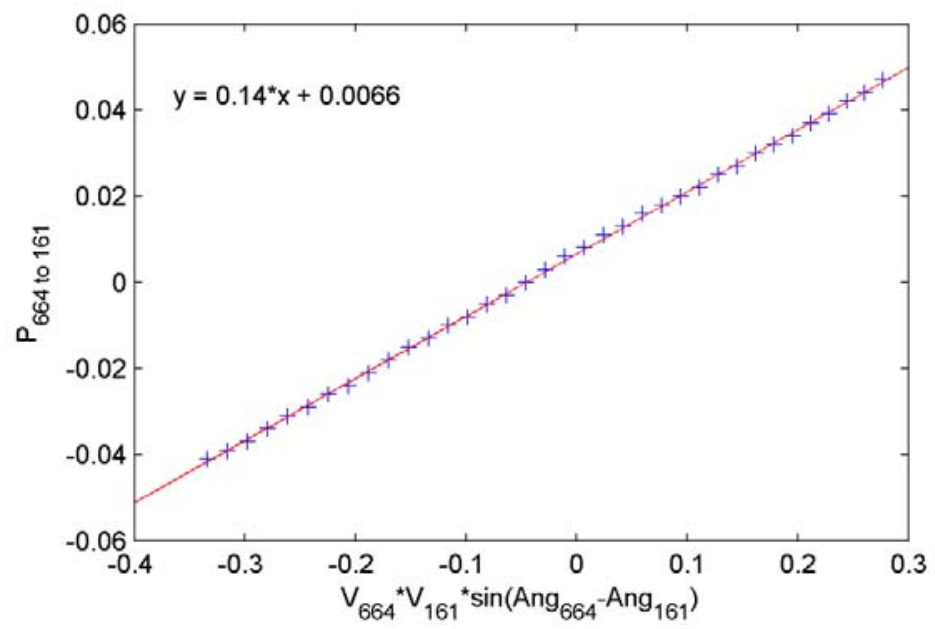

Figure 15: Plot of Power Flow into Boundary Station with Voltages and Angle Difference Multiplication for $345 \mathrm{kV}$ Boundary Station 


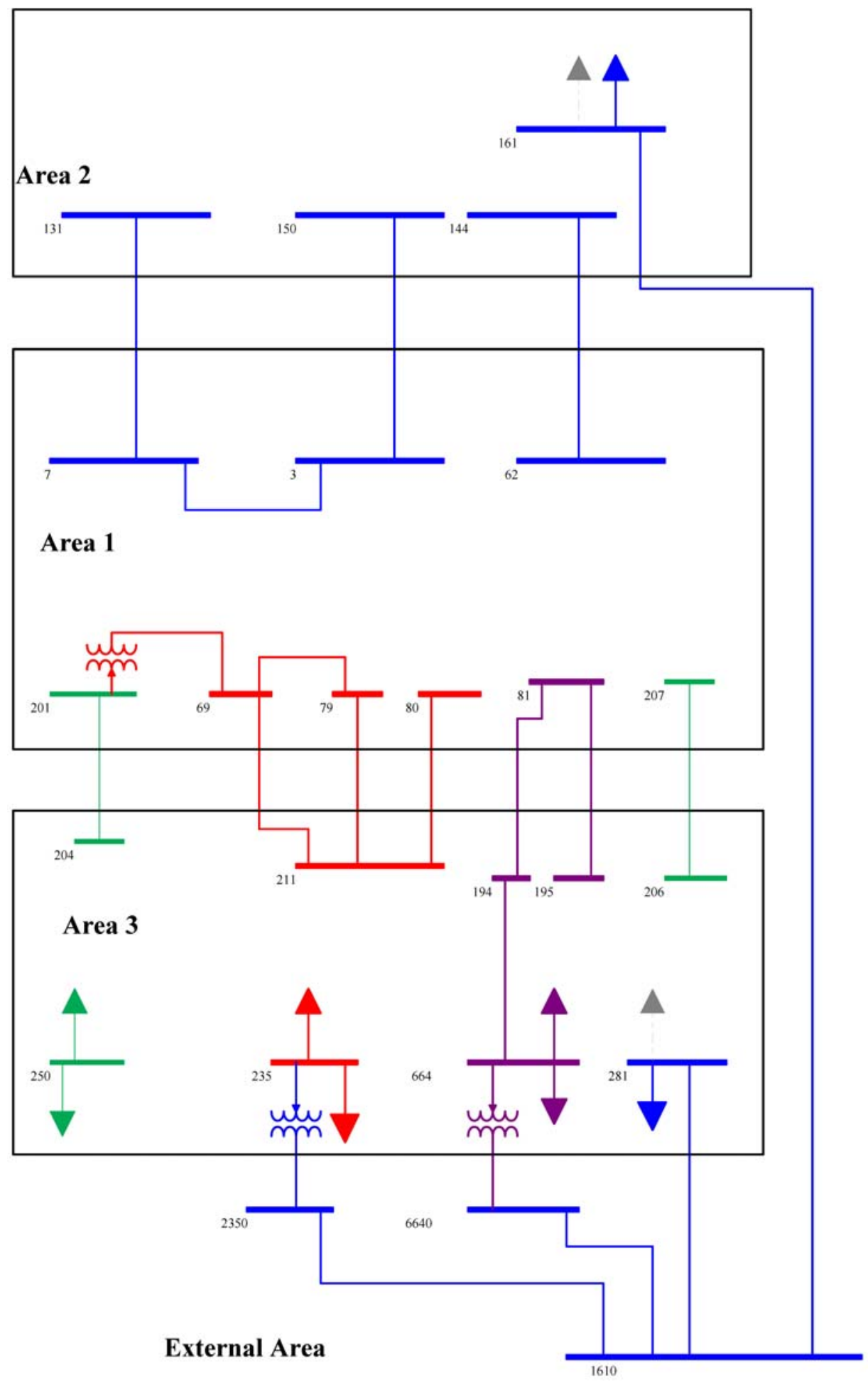

Figure 16: Four Area System with Equivalent Circuit 
Table 2: Equivalent Circuit Parameters and correlation coefficient

\begin{tabular}{|l|l|l|l|}
\hline & $\mathbf{1 3 8} \mathbf{~ k V}$ line & $\mathbf{2 3 0} \mathbf{~ k V}$ line & $345 \mathrm{kV}$ line \\
& $\mathbf{( 2 3 5 0 - 1 6 1 0 )}$ & $\mathbf{( 2 8 1 - 1 6 1 0 )}$ & $\mathbf{( 6 6 4 0 - 1 6 1 0 )}$ \\
\hline Equivalent impedance (p.u.) & 1.56 & 0.79 & 6.93 \\
\hline Correlation Coefficient & 0.9998 & 0.9999 & 0.9998 \\
\hline
\end{tabular}

\subsubsection{Boundary Matching and Model Merging}

As explained above, power utilities only have real-time data corresponding to their internal area. They do not have real-time data corresponding to the external area. Therefore, once the external equivalent circuit is connected to the internal model, it is necessary to carry out some form of boundary matching exercise to ensure that the internal state of the power system data does not change by adding the equivalent circuit. In real-time security assessment this is carried out in the state estimator. Consider the simple power system shown in Figure 17, where the internal system is connected to the external system via four boundary stations B1, B2, B3 and B4. In boundary stations B1 and B2, there are two internal lines and one external line, and in boundary stations B3 and B4 there is only one internal line and one external line each. Utilities normally have visibility of the boundary stations including flow coming in from the external area. Therefore, it can be assumed that all flows in the boundary stations, including flows to the external areas, are known. Now, according to the nodal equation and assuming that there is no power loss in the stations, the following equation can be written for active and reactive power entering and leaving station B1. 


$$
\left(\mathrm{P}_{1}+\mathrm{jQ} \mathrm{Q}_{1}\right)+\left(\mathrm{P}_{2}+\mathrm{jQ} \mathrm{Q}_{2}\right)=\mathrm{P}_{4}+\mathrm{jQ} \mathrm{Q}_{4}
$$

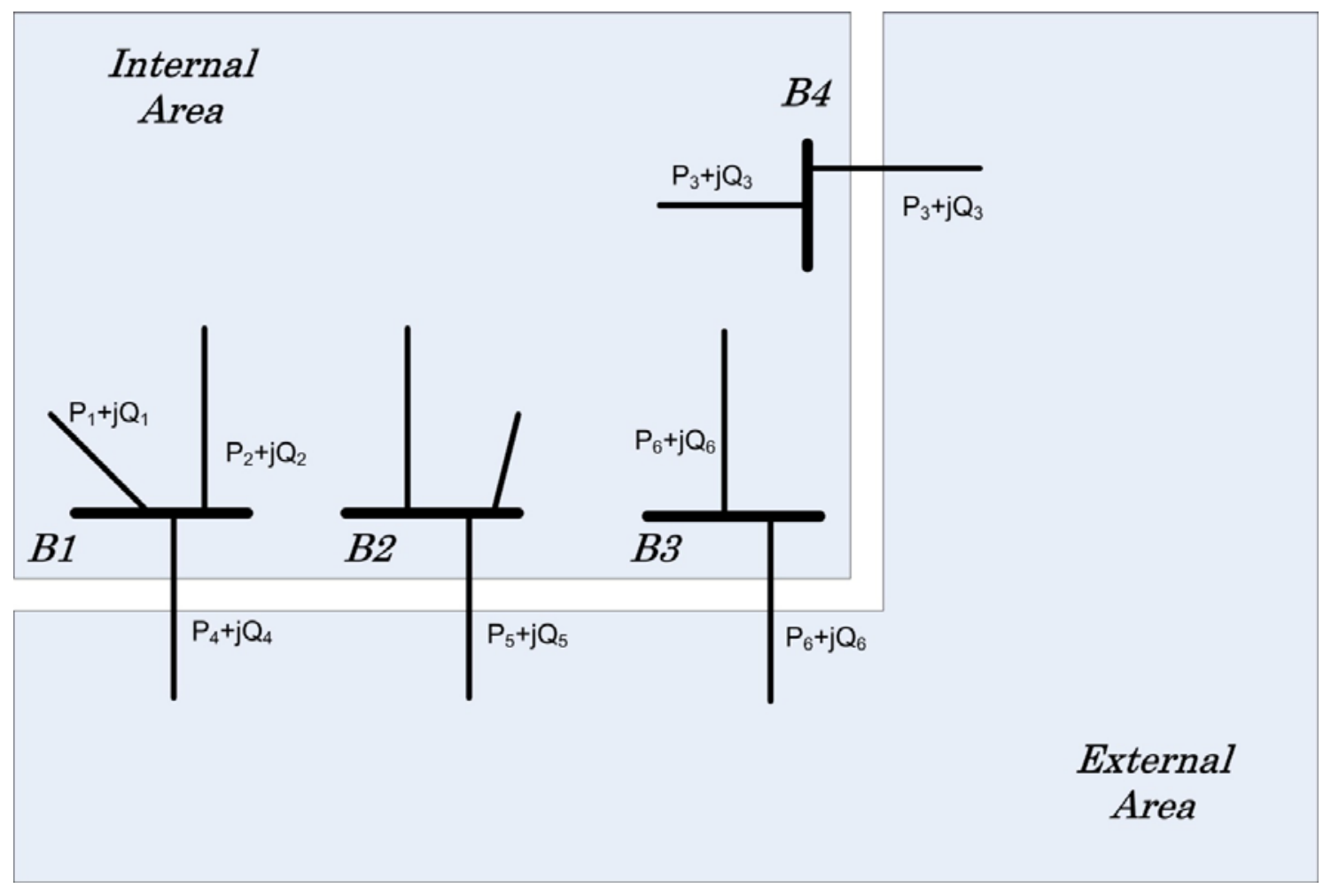

Figure 17: Internal System with External System

Consider the equivalent circuit prepared and it is necessary to join the equivalent circuit with the internal area. Also assume that the connection between the boundary station B2 and the equivalent circuit can be ignored. This is illustrated in Figure 18. 


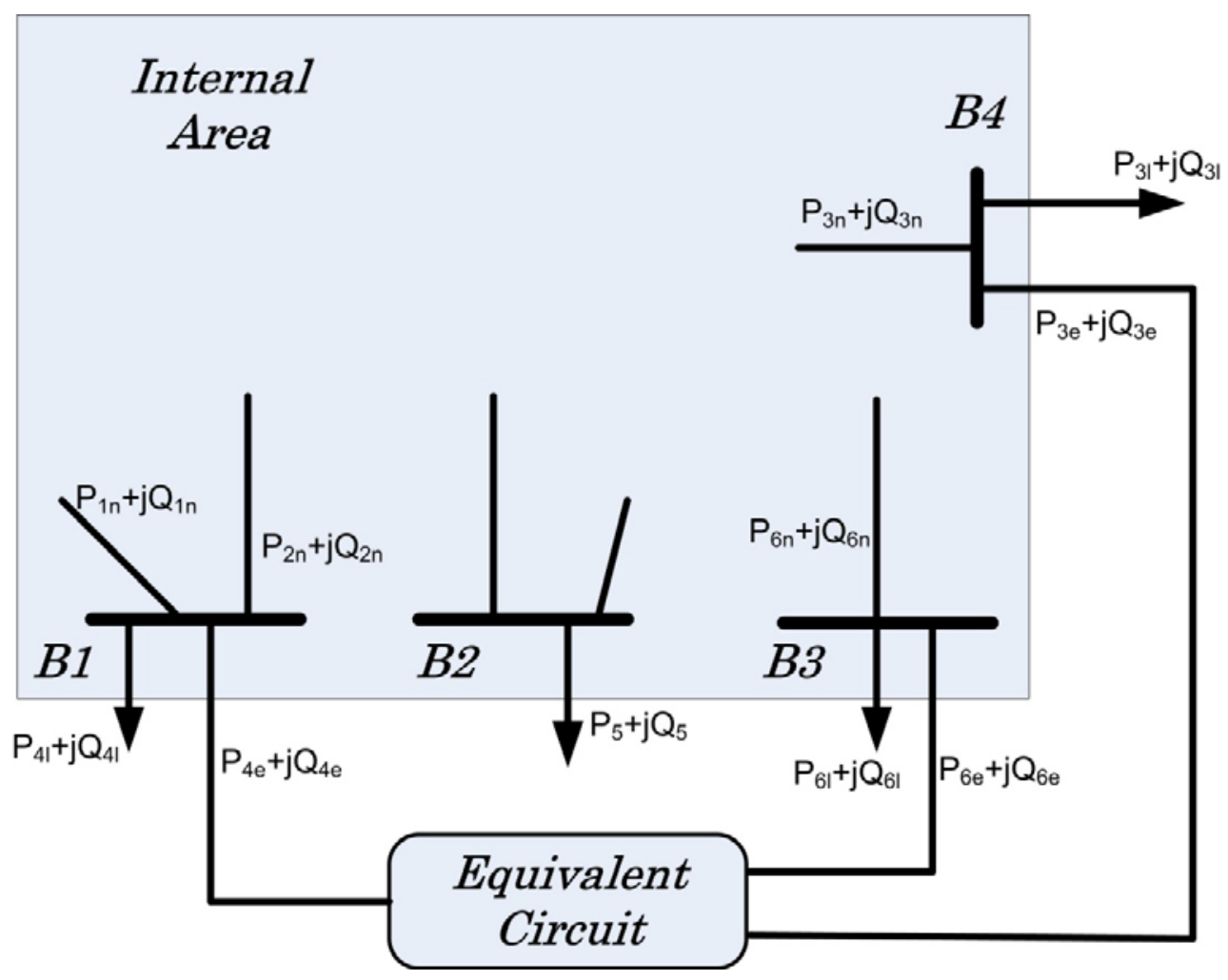

Figure 18: Internal System with Equivalent Circuit

Since, boundary station B2 is not connected to the equivalent network, the flow on the external line from the boundary station B2 can be modelled as a load in the station. Once the equivalent circuit is connected to the internal area, there will be a power flow between the internal area and the equivalent circuit as shown in the diagram. However, the connection of the equivalent circuit should not alter the flow on internal lines. This logic can be used to change the value of loads connected to the stations B1, B3 and B4. For an example, the value of the $\mathrm{P}_{41}$ and $\mathrm{Q}_{41}$ can be changed so that:

$$
\left(\mathrm{P}_{4 \ln }+j \mathrm{Q}_{4 \ln }\right)+\left(\mathrm{P}_{4 \mathrm{e}}+\mathrm{jQ} \mathrm{Q}_{4 \mathrm{e}}\right)=\left(\mathrm{P}_{1}+\mathrm{jQ} \mathrm{Q}_{1}\right)+\left(\mathrm{P}_{2}+\mathrm{jQ}_{2}\right)
$$


Similarly, load connected to bus B3 and B4 can be changed to provide the same internal flow as the original model. Once the load values are changed, it is necessary to resolve the power flow to obtain a valid solution. Resolving can change the flow from the equivalent circuit into the internal area, which forces a change in value to the load connected at the boundary. The process of changing load values and solving the power flow should be carried out iteratively until there is no difference in the flows towards the internal area from the boundary stations.

This approach was followed to come up with the boundary conditions for the internal area of the power system shown in the Figure 16. The equivalent circuit is connected to Area-3 at buses 250, 235, 281 and 664 and to Area-2 at bus 161. These are the boundary buses for the system under investigation. Since the power transfer from Bus 250 was small, it was ignored from the equivalent circuit and, the flow on line 250 to 761 was modelled as a load at station 250 . Load connected to boundary buses $235,281,664$ and 161 was iteratively changed until the same flows were injected from the boundary buses into the internal area.

Boundary matching is required when external equivalent circuit is connected to the internal area to ensure that adding external equivalent does not alter the internal state of the power system. 


\subsubsection{Validating Equivalent Circuit}

Equivalent circuit parameter calculation and boundary matching will provide a new base case with the equivalent circuit. It is necessary to validate the developed equivalent circuit. The purpose of developing an equivalent circuit was to use it in the security assessment of Area-1. Therefore, elements in Area-1, including tie lines between Area-2 and Area-3, were used as the contingencies and monitoring elements to validate the equivalent circuit. This provides 33 lines as contingencies and monitoring elements.

In model validation, it is often a good practice to compare the results of the analysis with other existing methods. Therefore, two additional equivalent circuits were developed by using different methods. It has been discussed that admittance reduction is one of the popular methods used in the industry. Commercially available software was used to develop an equivalent circuit using the impedance reduction method. The second equivalent circuit was developed ignoring the impact of the external circuit. In this scenario, power flows from the boundary buses into the external areas were modelled as loads in the boundary stations.

When selected contingencies were implemented in the full model, the post contingency power flow models failed to converge for five of the contingencies due to voltage collapse of the system. Since this is a system problem and not related to the equivalent circuit. These five contingencies were omitted from the analysis. The remaining 28 contingencies were also implemented into the three reduced models, which were created 
using (a) the proposed method(b) the impedance reduction method, and (c) by ignoring the external circuit in the reduced model. Combination of 28 contingencies with 33 monitoring lines for both active and reactive power produced 1848 data points for comparison. Results from the contingency analysis which includes real and reactive power on all the lines in Area-1 of the reduced models were then compared with contingency analysis of the full model. Summary of the analysis is shown in Table 3 and Table 4.

Table 3 presents the distribution of absolute error among the three methods. It can be seen that, proposed method reports 51 instances where the absolute error is between 1$2 \mathrm{MW} / \mathrm{MVAr}$. That number was 23 for the impedance reduction method and it was 119 when the impact of the reduced model was ignored. It can also be seen that the by looking at the error between 1-2 MW, impedance reduction method is better than the proposed method. It should be noted that, when solving the power flow, 1MW/MVAr was selected as the convergence tolerance according to the acceptable industry practice.

Table 3 also shows that, proposed method reports 13 instances or $0.7 \%$ where the absolute error is more than $2 \mathrm{MW} / \mathrm{MVAr}$ and impedance reduction method only shows 7 instances or $0.37 \%$ where as when the impact of equivalent circuit was ignored that number rose to 161 or $8.7 \%$ out of 81 instances or $4.4 \%$ were more than $5 \mathrm{MW} / \mathrm{MVAr}$. 
Table 3: Distribution of Absolute Error

\begin{tabular}{|l|l|l|l|l|l|}
\hline & \multicolumn{5}{l|}{ Number of occurrences when the absolute error in } \\
& MW/MVAr is \\
& $1-2$ & $2-3$ & $3-4$ & $4-5$ & $>5$ \\
\hline Proposed method & 51 & 9 & 3 & - & 1 \\
\hline Impedance reduction method & 23 & 7 & - & - & - \\
\hline Ignoring external circuit & 119 & 39 & 26 & 15 & 81 \\
\hline
\end{tabular}

Table 4: Results Comparisons Between Methods

\begin{tabular}{|l|l|l|}
\hline & $\begin{array}{l}\text { Average absolute error } \\
\text { (MW/MVAr) }\end{array}$ & $\begin{array}{l}\text { Highest absolute error } \\
\text { (MW/MVAr) }\end{array}$ \\
\hline Proposed method & 1.9 & 5.9 \\
\hline Impedance reduction method & 1.8 & 2.8 \\
\hline Ignoring external circuit & 5.6 & 28.4 \\
\hline
\end{tabular}

From Table 4, it can be seen that the highest error reported by the proposed method was 5.9 MW/MVAr where as the highest error reported by the impedance reduction method was 2.8 MW/MVAr. However, when the impact of the external circuit was ignored, the highest error increased to 28.4 MW/MVAr.

The average absolute error reported by the proposed method was 1.9 MW/MVAr, where as the average absolute error for the impedance reduction method was 1.8 
MW/MVAr. However, when the impact of the external circuit was ignored, the average error increased to $5.6 \mathrm{MW} / \mathrm{MVAr}$.

This analysis shows that the accuracy of the proposed method using the boundary bus information is very close to that of the admittance reduction method, which uses the information of the entire external system to create the equivalent circuit.

\subsection{Summary}

This chapter presented a novel method to develop equivalent circuit using available boundary information. Modified IEEE three area 300 bus system was used to demonstrate the equivalent circuit creation process and model validation.

Power system simulation was used to create transfer analysis between two areas to generate necessary data to create equivalent circuit. These data were then used to generate the equivalent circuit. Commercially available power system simulation software was used to generate another equivalent circuit based on the impedance reduction process. Another network was created ignoring the external area. All three models were subjected to the same contingency for the benchmarking purposes.

Benchmarking results revealed that proposed method provide accuracy comparable to the equivalent circuit generated using the impedance reduction method. 


\section{Chapter 4}

\section{Development of an Equivalent Circuit}

\section{using Least Square Method}

Previous chapter presented a method to generate equivalent circuit using boundary information. Even though, this method presents very good results, this method cannot be used to determine the impedance between the boundary stations. Therefore it may not produce acceptable results for some power systems. This chapter presents a more general method to calculate equivalent circuit parameters using available boundary information.

\subsection{Proposed Methodology}

Consider the power system shown in Figure 19 with the equivalent network. The equivalent network was created by connecting four boundary buses to each other. Volt- 
age and phase angle of boundary buses are $\mathrm{V}_{1} \angle \theta_{1}, \mathrm{~V}_{2} \angle \theta_{2}, \mathrm{~V}_{3} \angle \theta_{3}, \mathrm{~V}_{4} \angle \theta_{4}$ and power injection into each boundary bus is $\mathrm{P}_{1}+\mathrm{jQ}_{1}, \mathrm{P}_{2}+\mathrm{jQ}_{2}, \mathrm{P}_{3}+\mathrm{jQ} \mathrm{Q}_{3}, \mathrm{P}_{4}+\mathrm{jQ} \mathrm{Q}_{4}$ and load connected to each of the boundary bus is $\mathrm{PL}_{1}+\mathrm{jQL}_{1}, \mathrm{PL}_{2}+\mathrm{jQL}_{2}, \mathrm{PL}_{3}+\mathrm{jQL} \mathrm{L}_{3}, \mathrm{PL}_{4}+\mathrm{jQL} \mathrm{L}_{4}$.

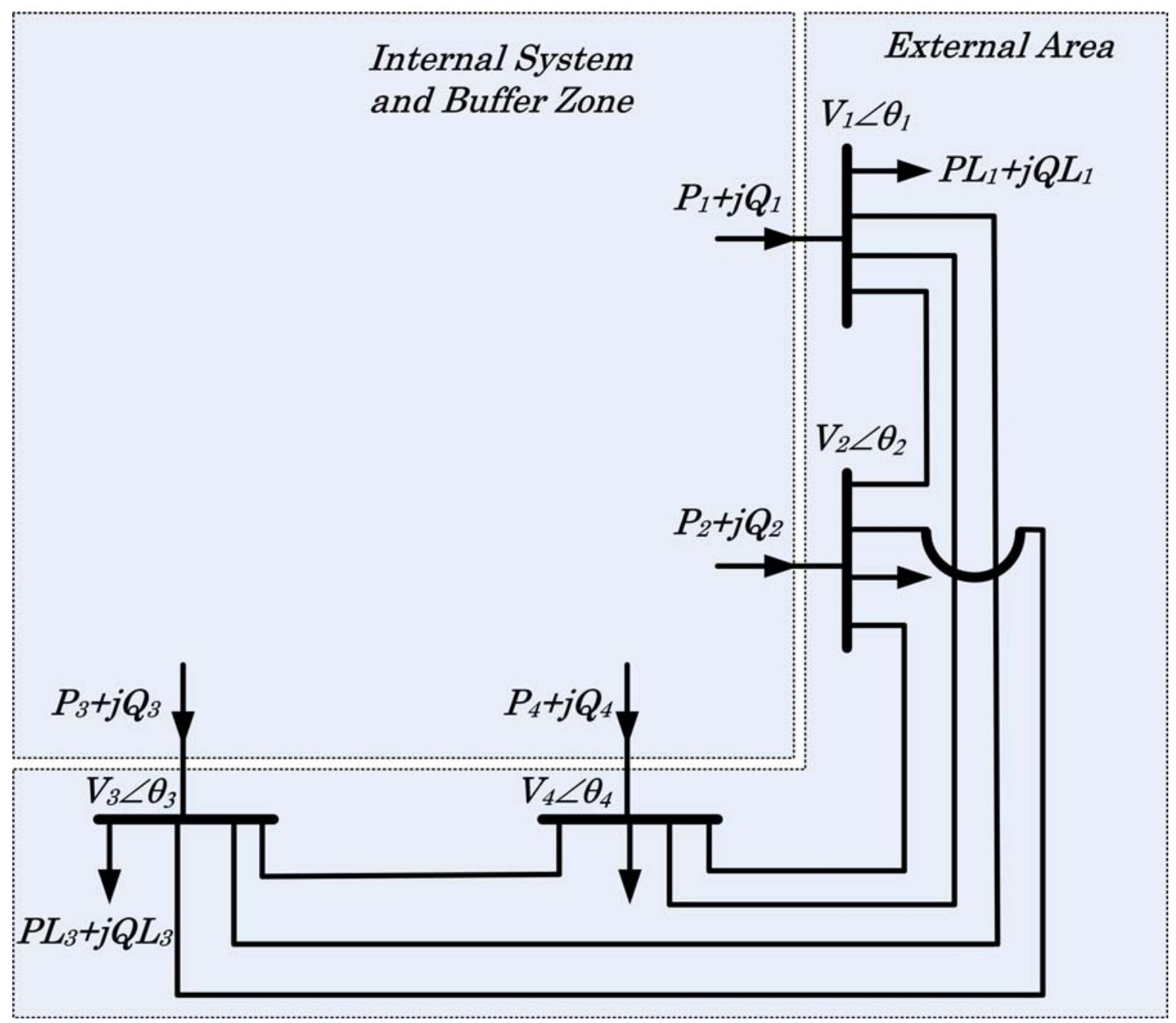

Figure 19: Power system network with equivalent network 
Following equation can be written for active power balance at bus 1 .

$$
P_{1}=P L_{1}+P_{12}+P_{13}+P_{14}
$$

Where $\mathrm{P}_{12}, \mathrm{P}_{13}, \mathrm{P}_{14}$ are the power transfer from bus 1 to bus 2 , bus 1 to bus 3 , and bus 1 to bus 4 .

The power transfer from bus 1 to bus 2 can be written as

$$
P_{12}=V_{1} V_{2}\left[G_{12} \cos \left(\theta_{1}-\theta_{2}\right)+B_{12} \sin \left(\theta_{1}-\theta_{2}\right)\right]
$$

Assuming that $G_{12}$ is small compared to $B_{12}$ for a transmission network, the above equation can be re-written as

$$
P_{12}=V_{1} V_{2} B_{12} \sin \left(\theta_{1}-\theta_{2}\right)
$$

Similarly power transfer from bus 1 to $3\left(\mathrm{P}_{13}\right)$ and bus 1 to 4 also can be written as:

$$
\begin{aligned}
& P_{13}=V_{1} V_{3} B_{13} \sin \left(\theta_{1}-\theta_{3}\right) \\
& P_{14}=V_{1} V_{4} B_{14} \sin \left(\theta_{1}-\theta_{4}\right)
\end{aligned}
$$

Now the equation (9) can be written again as:

$$
P_{1}=V_{1} V_{2} B_{12} \sin \left(\theta_{1}-\theta_{2}\right)+V_{1} V_{3} B_{13} \sin \left(\theta_{1}-\theta_{3}\right)+V_{1} V_{4} B_{14} \sin \left(\theta_{1}-\theta_{2}\right)+P L_{1}
$$

Similar equations can be written for power coming into boundary bus $2\left(\mathrm{P}_{2}\right)$ and boundary bus $3\left(\mathrm{P}_{3}\right)$.

$$
\begin{aligned}
& P_{2}=-V_{1} V_{2} B_{12} \sin \left(\theta_{1}-\theta_{2}\right)+V_{2} V_{3} B_{23} \sin \left(\theta_{2}-\theta_{3}\right)+V_{2} V_{4} B_{24} \sin \left(\theta_{2}-\theta_{4}\right)+P L_{2} \\
& P_{3}=-V_{1} V_{3} B_{13} \sin \left(\theta_{1}-\theta_{3}\right)-V_{2} V_{3} B_{23} \sin \left(\theta_{2}-\theta_{3}\right)+V_{3} V_{4} B_{34} \sin \left(\theta_{3}-\theta_{4}\right)+P L_{3}
\end{aligned}
$$


These equations can be written in Matrix form as:

$$
\left[H_{1}, I_{3}\right] X=Y_{1}
$$

Where:

$$
\begin{aligned}
& H 1=\left[\begin{array}{ccc}
V_{1} V_{2} \sin \left(\theta_{1}-\theta_{2}\right) & V_{1} V_{3} \sin \left(\theta_{1}-\theta_{3}\right) & V_{1} V_{4} \sin \left(\theta_{1}-\theta_{4}\right) \\
-V_{1} V_{2} \sin \left(\theta_{1}-\theta_{2}\right) & 0 & 0 \\
0 & -V_{1} V_{3} \sin \left(\theta_{1}-\theta_{3}\right) & 0
\end{array}\right. \\
& \left.\begin{array}{ccc}
0 & 0 & 0 \\
V_{2} V_{3} \sin \left(\theta_{2}-\theta_{3}\right) & V_{2} V_{4} \sin \left(\theta_{2}-\theta_{4}\right) & 0 \\
-V_{2} V_{3} \sin \left(\theta_{2}-\theta_{3}\right) & 0 & V_{3} V_{4} \sin \left(\theta_{3}-\theta_{4}\right)
\end{array}\right] \\
& Y_{1}=\left[\begin{array}{l}
P_{1} \\
P_{2} \\
P_{3}
\end{array}\right] \\
& X=\left[\begin{array}{l}
B_{12} \\
B_{13} \\
B_{14} \\
B_{23} \\
B_{24} \\
B_{34} \\
P L_{1} \\
P L_{2} \\
P L_{3}
\end{array}\right]
\end{aligned}
$$

and $\mathrm{I}_{3}$ is a 3 by 3 identity matrix.

When the system condition changes, the value of boundary bus voltages, boundary bus angle and power injection will change. Each set of measurement can be used to generate a new set of $\mathrm{H}$ and $\mathrm{Y}$ matrices. These $\mathrm{H}$ and $\mathrm{Y}$ matrices can then be used to generate a system of over determined linear equations as shown in equation 20 below. The 
least square solution of these equations will provide the equivalent circuit impedances and boundary loads.

$$
\left[\begin{array}{cc}
H_{1} & I_{3} \\
H_{2} & I_{3} \\
& \\
H_{n-1} & I_{3} \\
H_{n} & I_{3}
\end{array}\right] X=\left[\begin{array}{c}
Y_{1} \\
Y_{2} \\
\\
Y_{n-1} \\
Y_{n}
\end{array}\right]
$$

\subsection{Developing an Equivalent Circuit}

Four area test system derived from IEEE three area bus system discussed in section 3.2 is used for the model validation. Four area system shown in Figure 11 is shown below again as Figure 20 for easy reference. 


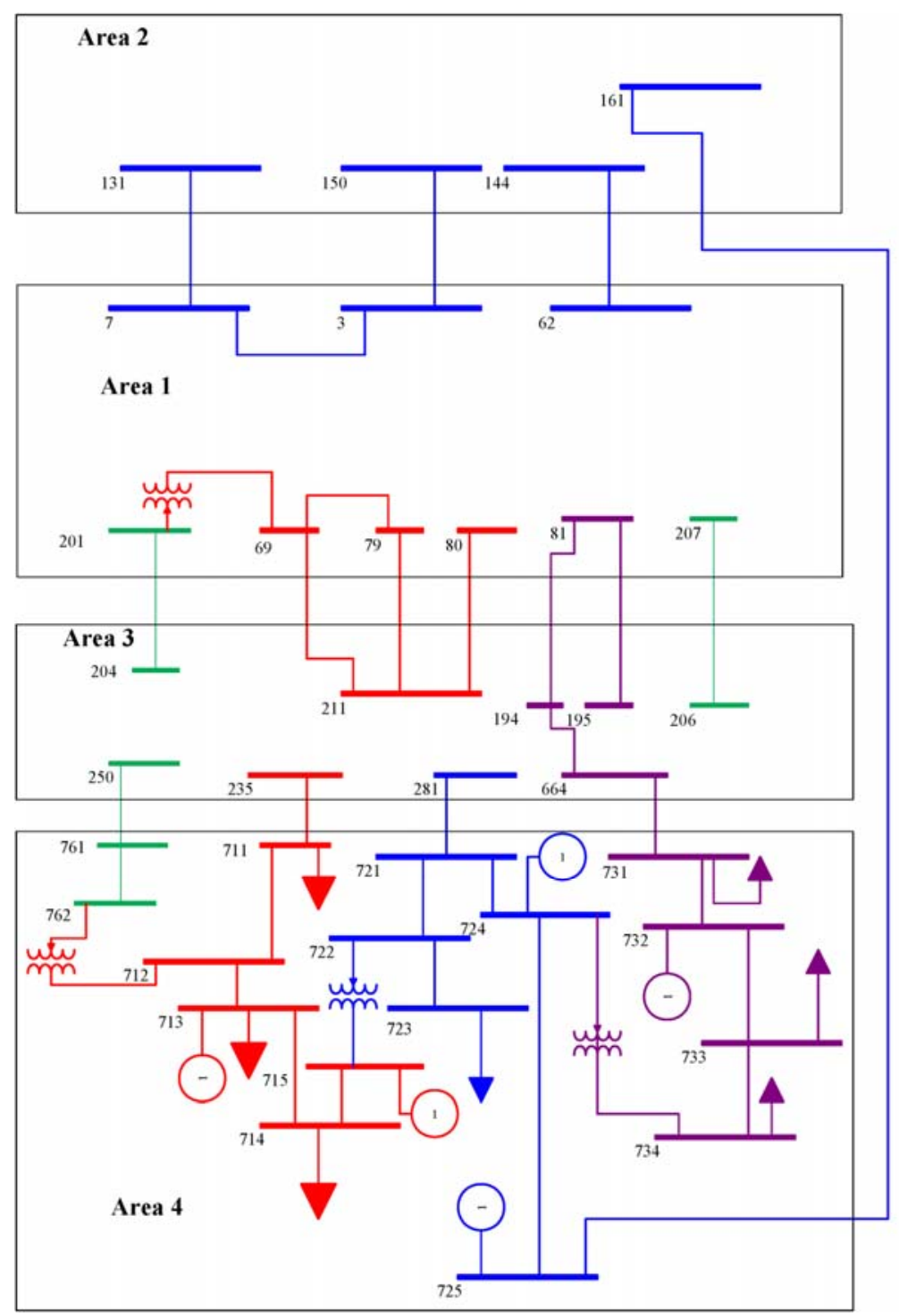

Figure 20: Four Area System

The same set of test data discussed in 3.2 was used in developing equivalent circuit. To compare the least square method with the linear regression method, equivalent circuit was first developed only connecting bus 235,664 and 281 to bus 161 as shown in Figure 21. The equivalent circuit impedance obtained for three lines using least square method was identical to the linear regression method. 


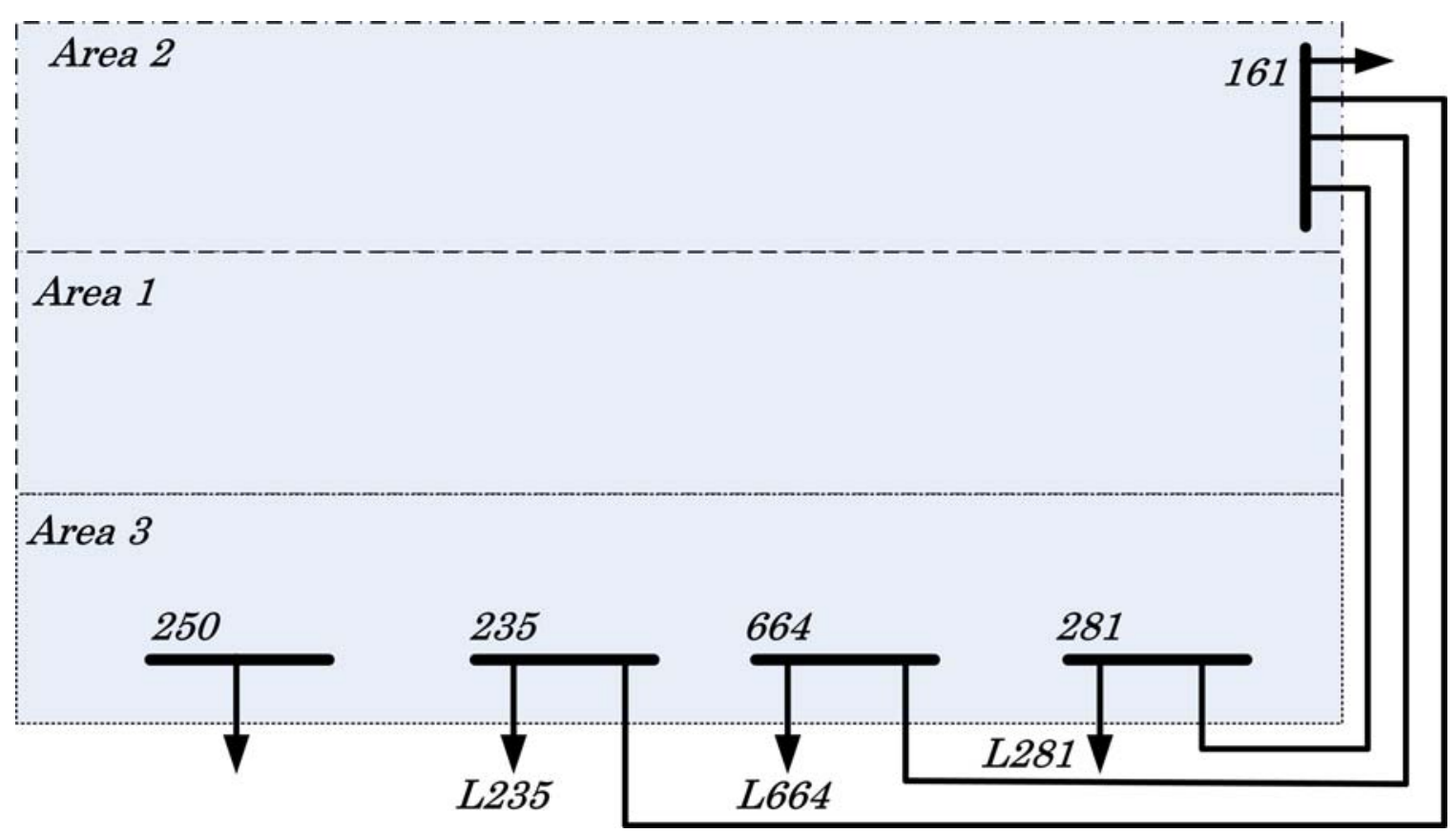

Figure 21: Simplified Equivalent Circuit

Least square method was then extended to calculate all the equivalent impedance parameters for the four area test system. Five boundary stations will provide 10 connections between stations as shown in Figure 22 . Equivalent circuit impedances calculated for the full network are shown in Table 5. 


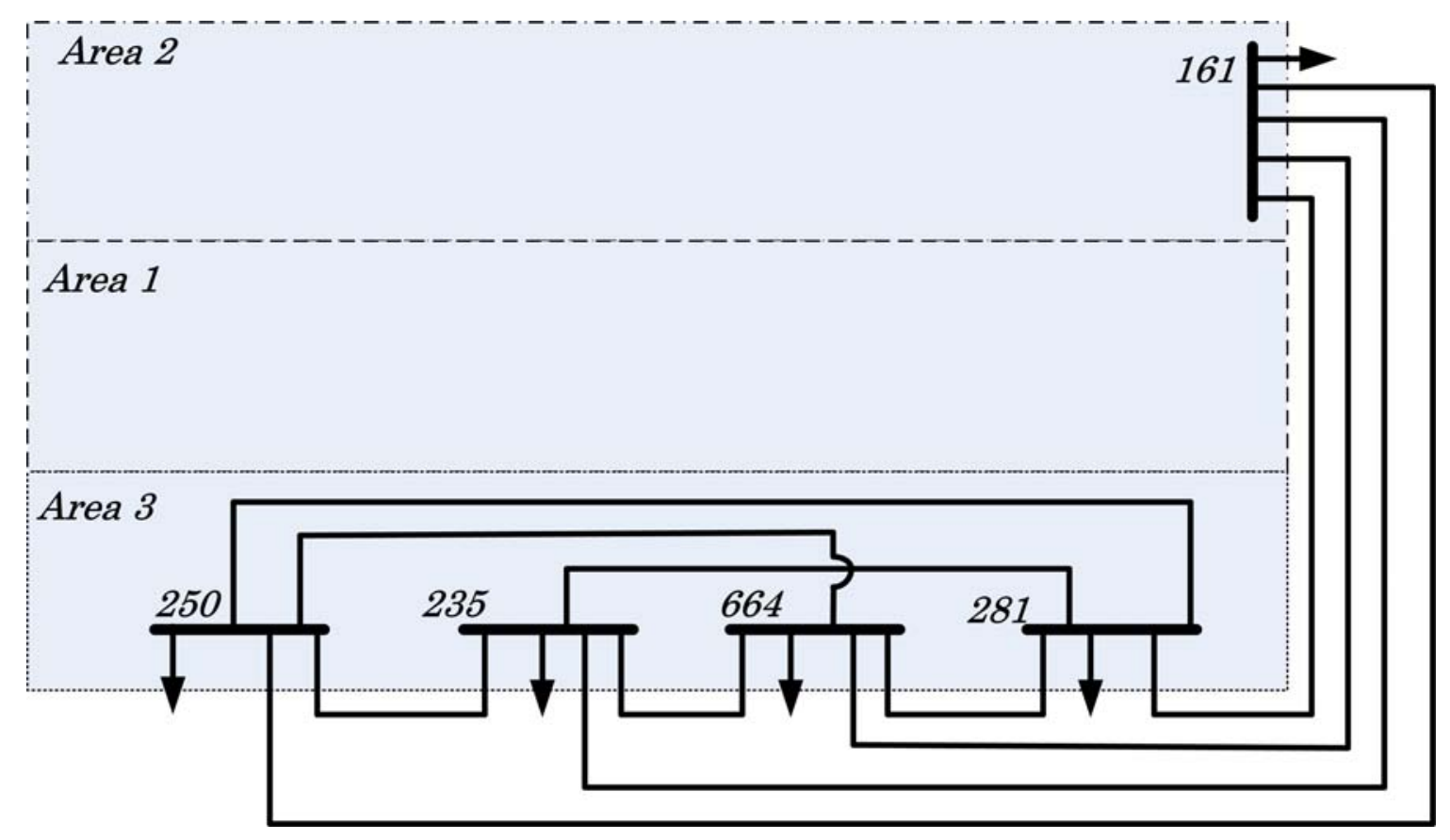

Figure 22: Equivalent circuit showing all the connections 
Table 5: Equivalent circuit parameters

\begin{tabular}{|l|l|}
\hline Line & $\begin{array}{l}\text { Impedance } \\
\text { (p.u) }\end{array}$ \\
\hline $250-235$ & 0.14 \\
\hline $250-664$ & -0.19 \\
\hline $250-281$ & -0.11 \\
\hline $250-161$ & 0.93 \\
\hline $235-664$ & 0.26 \\
\hline $235-281$ & -0.39 \\
\hline $235-161$ & -3.43 \\
\hline $664-281$ & -0.22 \\
\hline $664-161$ & -0.22 \\
\hline $281-161$ & 0.69 \\
\hline
\end{tabular}

It can be seen that some of the equivalent circuit impedances are negative. However, in a real transmission network, the transmission line reactances are positive unless the line is heavily compensated with series capacitors. It was also found that, when it attempts to generate a power flow with these parameters, power flow becomes unstable. This indicates, even though the least square method provide solution to a set of over determined linear equations, the answers of that solutions may not be practical. Therefore, it is necessary to apply additional constraints in to the least square method. 


\subsubsection{Applying Additional Constraints}

Least square problem can be modelled by adding inequality constraints to limit the impedance to have only positive value. The more details on this method can be found in literature $[157,158]$. Matlab built in functions can be used to determine the solutions to set of overdetermined system of linear equation in a given range.

The least square method was formulated to only provide positive impedance as the transmission line impedances. The method was first tested by calculating only three equivalent impedances between bus 235, 281 and 664 in Area- 3 with bus 161 in the Area-2 as shown in Figure 21. This method also produced the same value for the impedance as the method-I (Linear regression method). Least square method was then used to calculate the impedance between all the paths as shown in Figure 22. The results of the analysis are shown in Table 6. 
Table 6: Equivalent circuit impedance

\begin{tabular}{|l|l|}
\hline Line & $\begin{array}{l}\text { Impedance } \\
\text { (p.u) }\end{array}$ \\
\hline $250-235$ & 58.86 \\
\hline $250-664$ & 833.16 \\
\hline $250-281$ & $1.17 \times 10^{14}$ \\
\hline $250-161$ & $3.61 \times 10^{4}$ \\
\hline $235-664$ & 7.23 \\
\hline $235-281$ & 432.16 \\
\hline $235-161$ & 1.62 \\
\hline $664-281$ & 3.25 \\
\hline $664-161$ & 4.88 \\
\hline $281-161$ & 0.80 \\
\hline
\end{tabular}

It can be seen that links between 250-664, 250-281, 250-161 and 235-281 have very high impedance values. Therefore, it was decided to ignore those links from the equivalent circuit analysis and carry out the equivalent circuit analysis process again using the least square method. The new connections between the buses are shown in Figure 23 and the results of the analysis are shown in Table 7. 


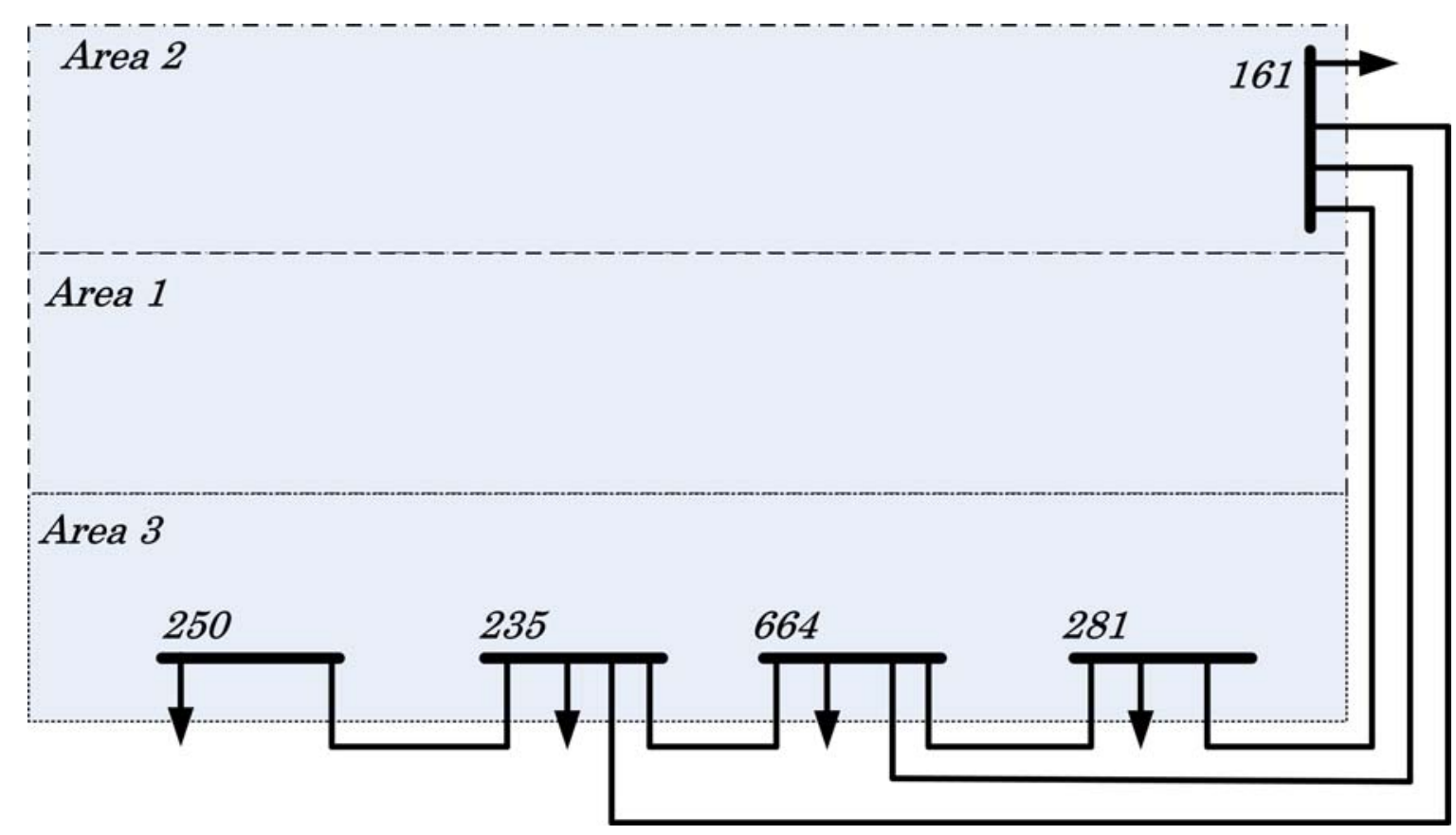

Figure 23 : Equivalent circuit connections

Table 7: Equivalent circuit parameters

\begin{tabular}{|l|l|}
\hline Line & $\begin{array}{l}\text { Impedance } \\
\text { (p.u) }\end{array}$ \\
\hline $250-235$ & 1.88 \\
\hline $235-664$ & 6.40 \\
\hline $235-161$ & 1.73 \\
\hline $664-281$ & 3.28 \\
\hline $664-161$ & 4.85 \\
\hline $281-161$ & 0.80 \\
\hline
\end{tabular}




\subsection{Results and Benchmarking}

The objective of developing an equivalent circuit is for security assessment for the internal system (Area-1). A number of benchmarking exercises are carried out to evaluate the accuracy of the developed equivalent circuit shown in Figure 23 with the security assessment in mind. This section presents the results of those analyses.

\subsubsection{Contingency Analysis}

The accuracy of the equivalent circuit was first analyzed by performing contingency analysis. Loss of single elements in Area-1 including tie lines with Area-2 and Area-3 were selected as the contingencies. The post contingency active and reactive power flow in all the branches in Area-1 were noted. These post contingency flows were then compared for the full model and the reduced model. The results of the analysis are shown in the Table 8. Note that this is the extension of Table 4 shown in section 3.2.2. 
Table 8: Results comparison

\begin{tabular}{|l|l|l|}
\hline & $\begin{array}{l}\text { Average absolute error } \\
\text { (MW/MVAr) }\end{array}$ & $\begin{array}{l}\text { Highest absolute error } \\
\text { (MW/MVAr) }\end{array}$ \\
\hline Linear regression method & 2.0 & 5.9 \\
\hline Impedance reduction method & 1.8 & 2.8 \\
\hline Ignoring external circuit & 5.6 & 28.4 \\
\hline Least square method & 2.0 & 3.6 \\
\hline
\end{tabular}

By comparing results, it can be seen that when more links were added to this particular network using the least square method, it produced slightly better results than linear regression method.

\subsubsection{Transfer Analysis}

The accuracy of the equivalent circuit was then analyzed by performing two transfer analyses. Transfer analyses were carried out between Area-1 and Area-2 as well as between Area-1 and Area-3. The transfer analysis was carried out by increasing generation in one area while increasing the load in the second area by a pre determined MW level. At each transfer level, security analysis was performed by simulating all the contingencies in Area-1 while observing voltage violation, thermal overload and voltage stability. This process is illustrated in Figure 24. In the security assessment, voltage violations were defined as deviations of bus voltages after the disturbance by more than $10 \%$ 
of the pre contingency voltage and voltage stability was defined as the inability to converge post contingency power flow.

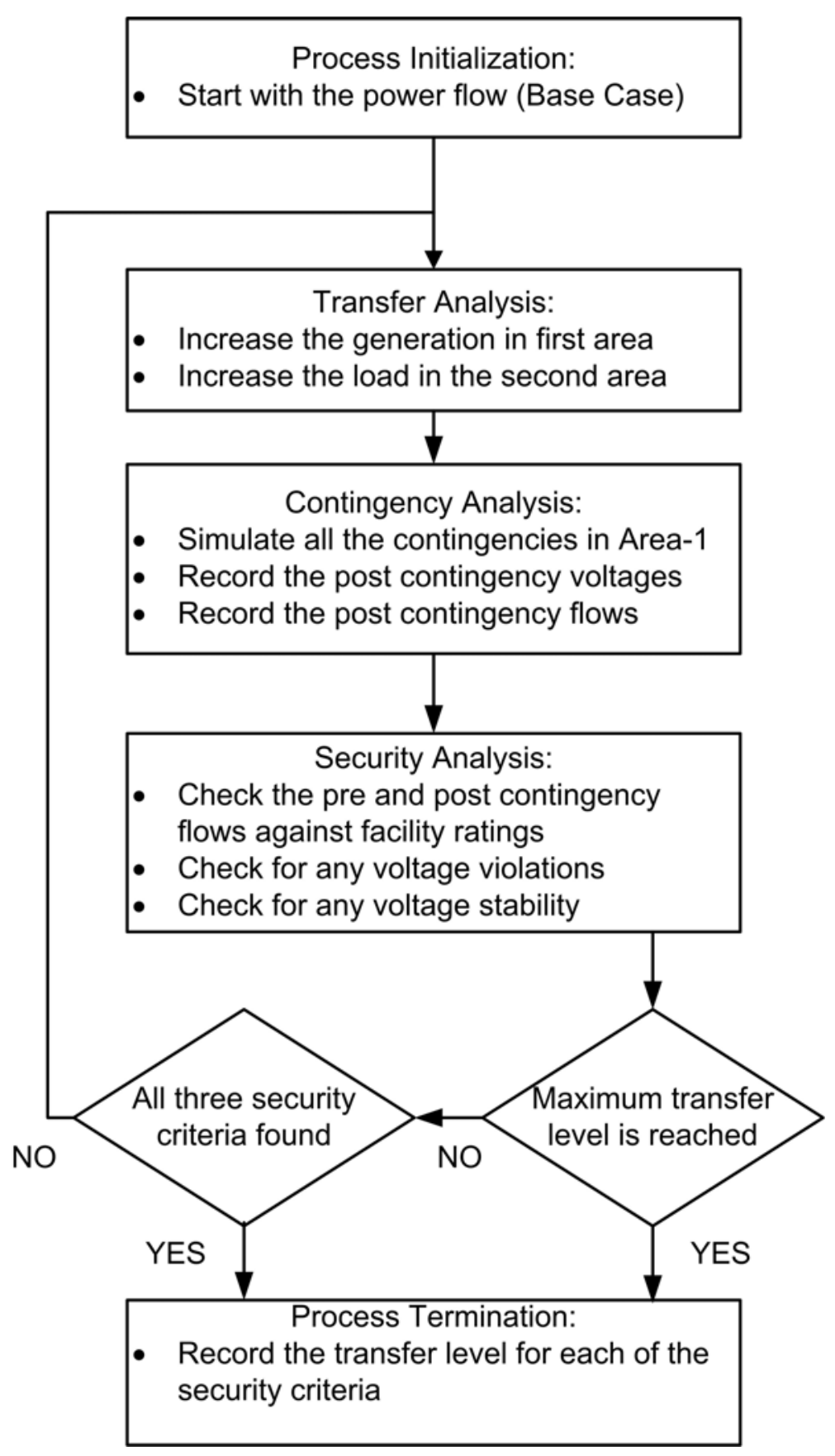

Figure 24: Security Analysis Process 
Power transfer level in which each of the security criteria was first observed for the full model and reduced model for Are-1 to Area-3 power transfer is shown in Table 9 and Table 10. Critical disturbance is the disturbance in which particular security constraints observed. Critical element is the element in which security constrained was observed. For an example, first thermal overload was observed at transfer level of 163.1 MW in full model and 165.3 MW in reduced model on branch 99-108 for contingent loss of branch 85-86. Similarly first voltage violation was observed at transfer level of 145.2 MW in the full model and 142.9 MW for reduced model at bus 63 and 526 for contingent loss of 46-81 and first voltage collapse was observed at transfer level of 149.7 MW in full model and 151.9 MW for the reduced model for contingent loss of branch 46-81.

Table 9: Transfer level Area 1 to Area 3: Full model

\begin{tabular}{|l|l|l|l|}
\hline Security Criteria & Transfer Level & Critical Disturbance & Critical Element \\
\hline Thermal Overload & 163.1 & $85-86$ & $99-108$ \\
\hline Voltage violation & 145.2 & $46-81$ & Bus 63 \& 526 \\
\hline Voltage stability & 149.7 & $46-81$ & - \\
\hline
\end{tabular}


Table 10: Transfer level Area 1 to Area 3: Reduced Model

\begin{tabular}{|l|l|l|l|}
\hline Security Criteria & Transfer Level & Critical Disturbance & Critical Element \\
\hline Thermal Overload & 165.3 & $85-86$ & $99-108$ \\
\hline Voltage violation & 142.9 & $46-81$ & Bus 63 \& 526 \\
\hline Voltage stability & 151.9 & $46-81$ & - \\
\hline
\end{tabular}

Table 11 and Table 12 show the transfer analysis results for the Area-1 to Area-2. In this transfer analysis scenario, voltage violations were not observed until the maximum transfer analysis is carried out in both models and the first thermal violation was observed in a pre contingency power flow in branch 7-131.

Table 11: Transfer level Area 1 to Area 2: Full model

\begin{tabular}{|l|l|l|l|}
\hline Security Criteria & Transfer Level & Critical Disturbance & Critical Element \\
\hline Thermal Overload & 383.7 & Pre & $7-131$ \\
\hline Voltage violation & - & - & - \\
\hline Voltage stability & 484.6 & $3-150$ & - \\
\hline
\end{tabular}

Table 12: Transfer level Area 1 to Area 2: Reduced Model

\begin{tabular}{|l|l|l|l|}
\hline Security Criteria & Transfer Level & Critical Disturbance & Critical Element \\
\hline Thermal Overload & 380.8 & Pre & $7-131$ \\
\hline Voltage violation & - & - & - \\
\hline Voltage stability & 482.3 & $3-150$ & - \\
\hline
\end{tabular}


By looking at transfer analysis results, it can be seen that equivalent circuit produced transfer level very close to the full model for both transfer analyses. They also produced same critical disturbance and critical element which is also very important.

\subsubsection{PV Analysis}

The accuracy of the equivalent circuit was also analyzed by performing PV analyses. Transfer analysis between Area-1 and Area-3 was selected for the PV analysis. Four buses in $230 \mathrm{kV}$ and $345 \mathrm{KV}$ voltage level which produced the highest voltage drop during the transfer analysis were selected for the P-V Analysis. Pre and post contingency voltage variation of those buses with the transfer level for contingency 46-81 is shown in Figure 25 to Figure 32. Contingency 46-81 was selected for this analysis as this contingency produced the voltage violation and voltage stability during the transfer analysis and assumed to have greater impact to the voltage of the studied area. 


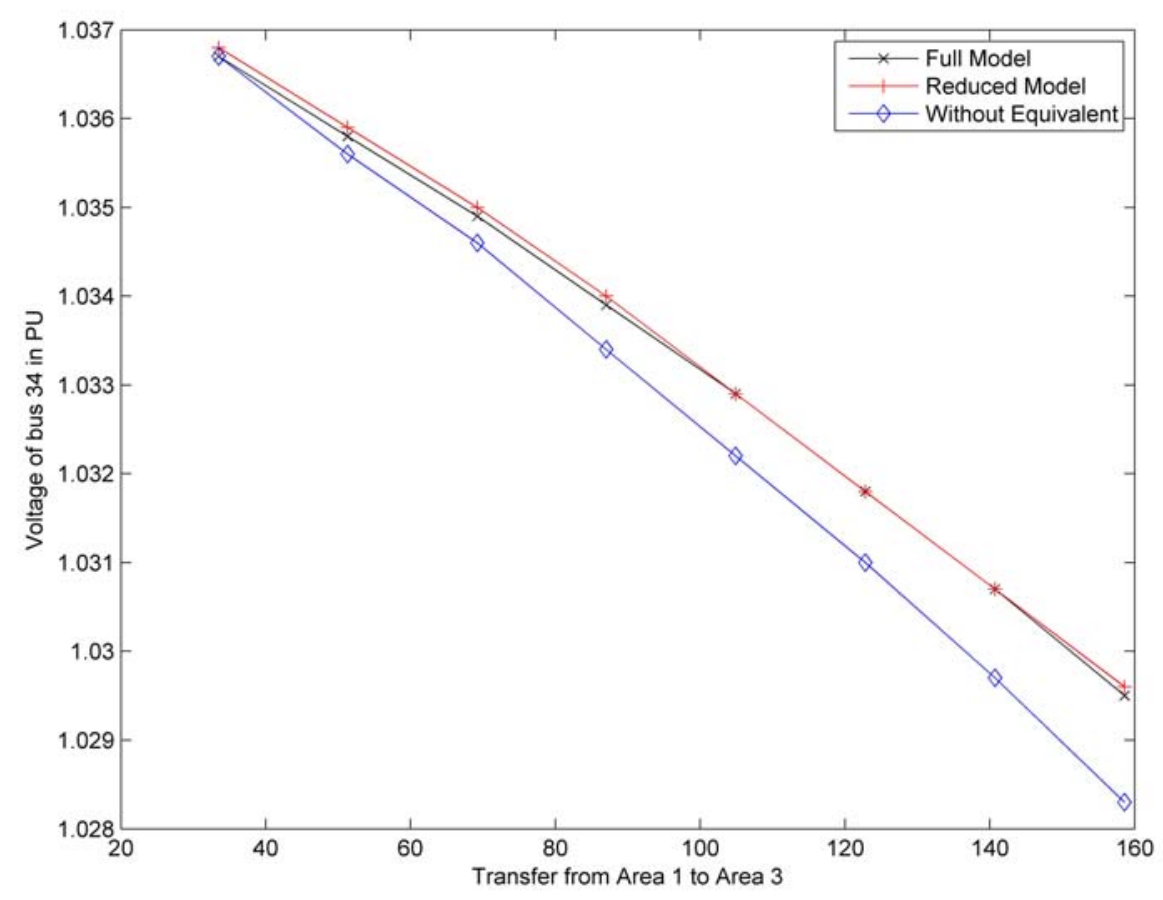

Figure 25: Pre Contingency voltage variation of bus 34 with transfer level

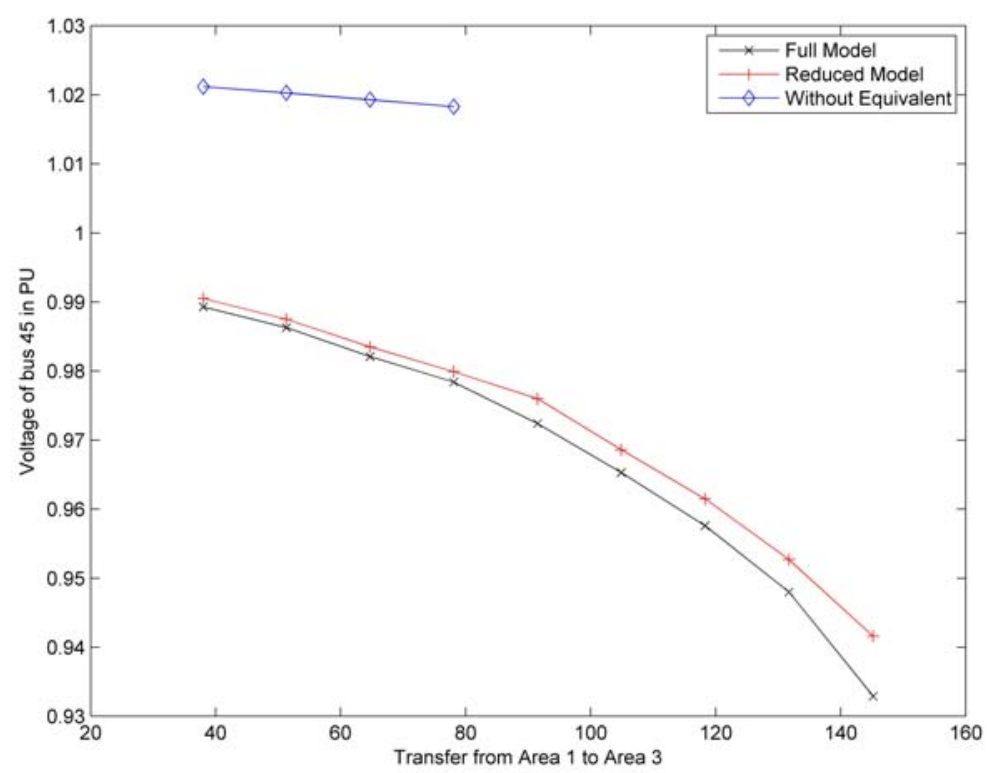

Figure 26: Post Contingency voltage variation of bus 34 with transfer level 


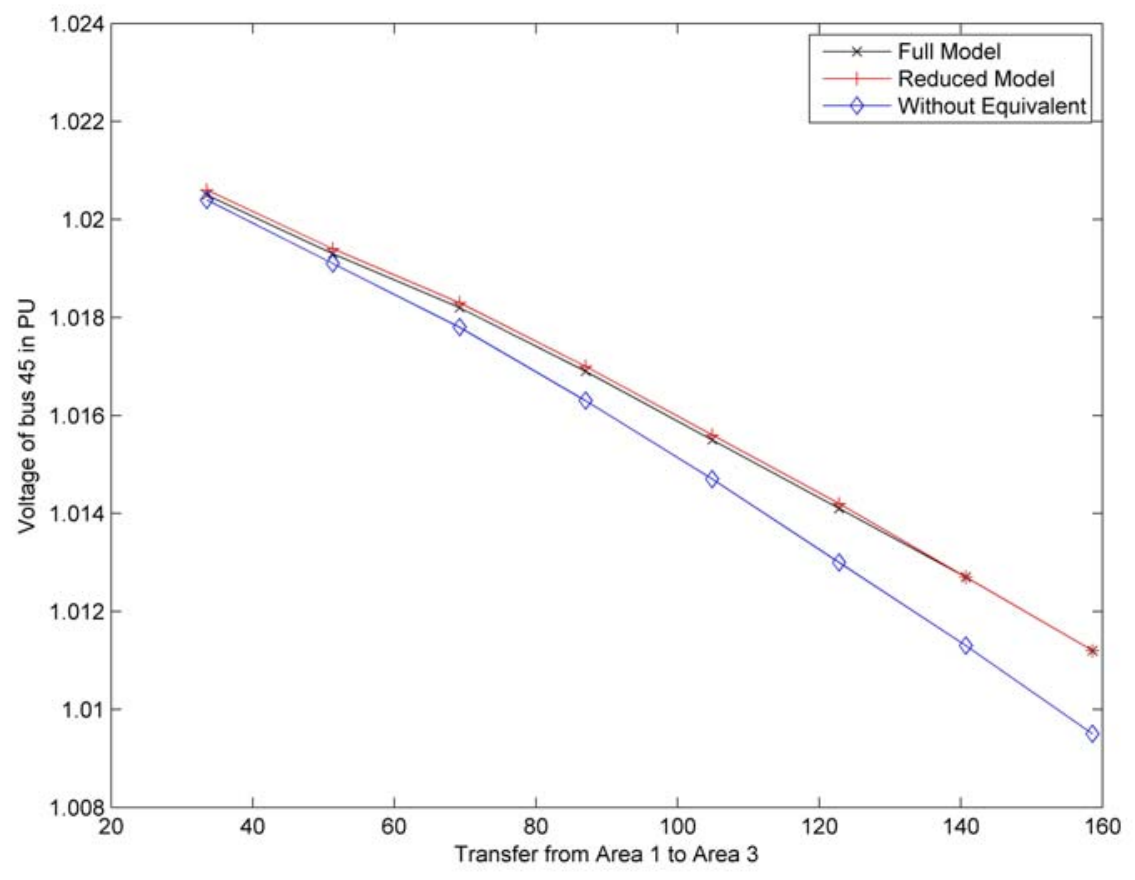

Figure 27: Pre Contingency voltage variation of bus 45 with transfer level

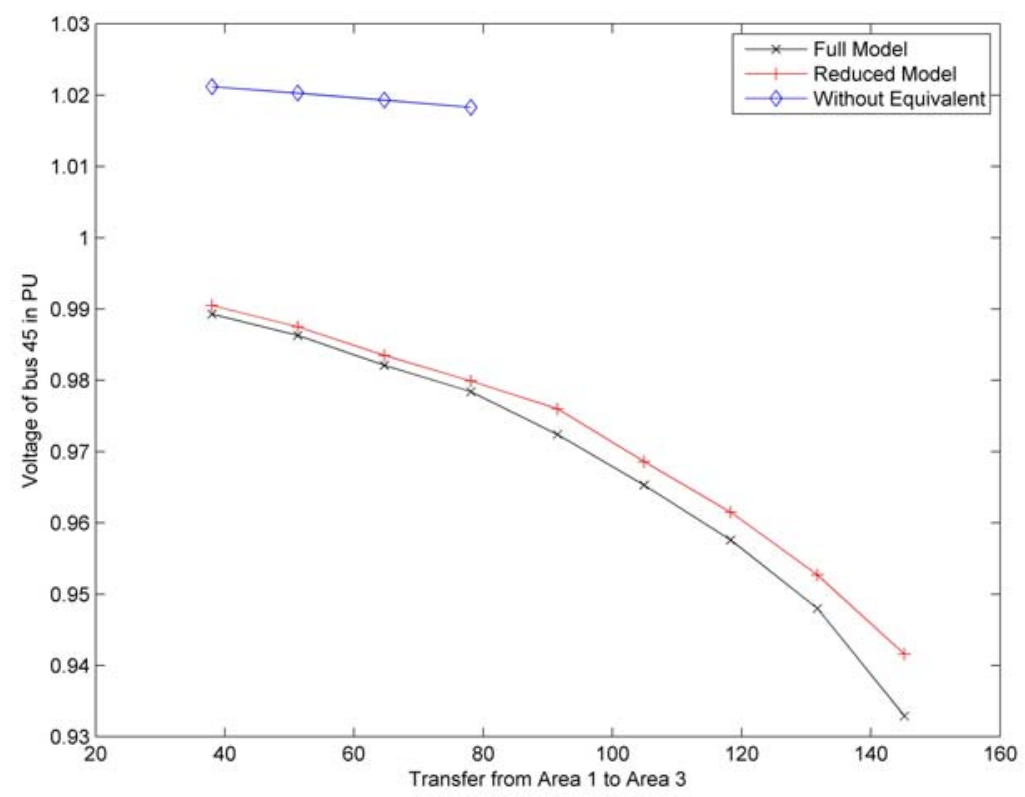

Figure 28: Post Contingency voltage variation of bus 45 with transfer level 


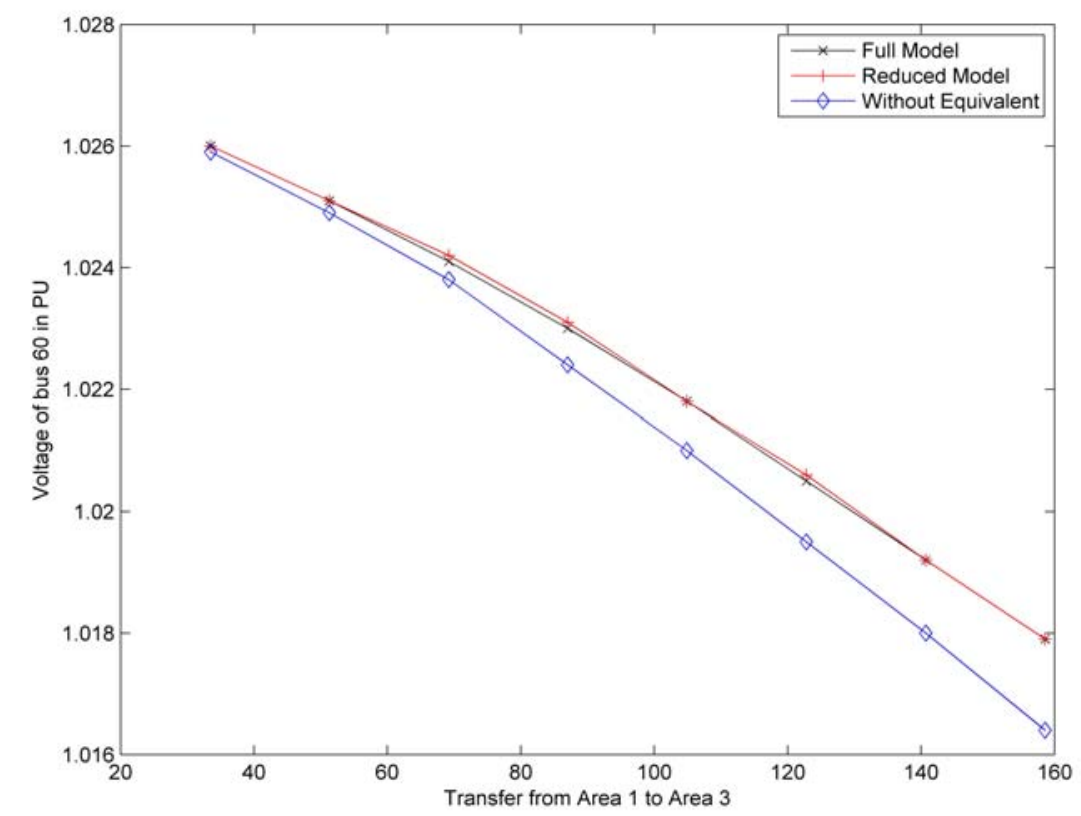

Figure 29: Pre Contingency voltage variation of bus 60 with transfer level

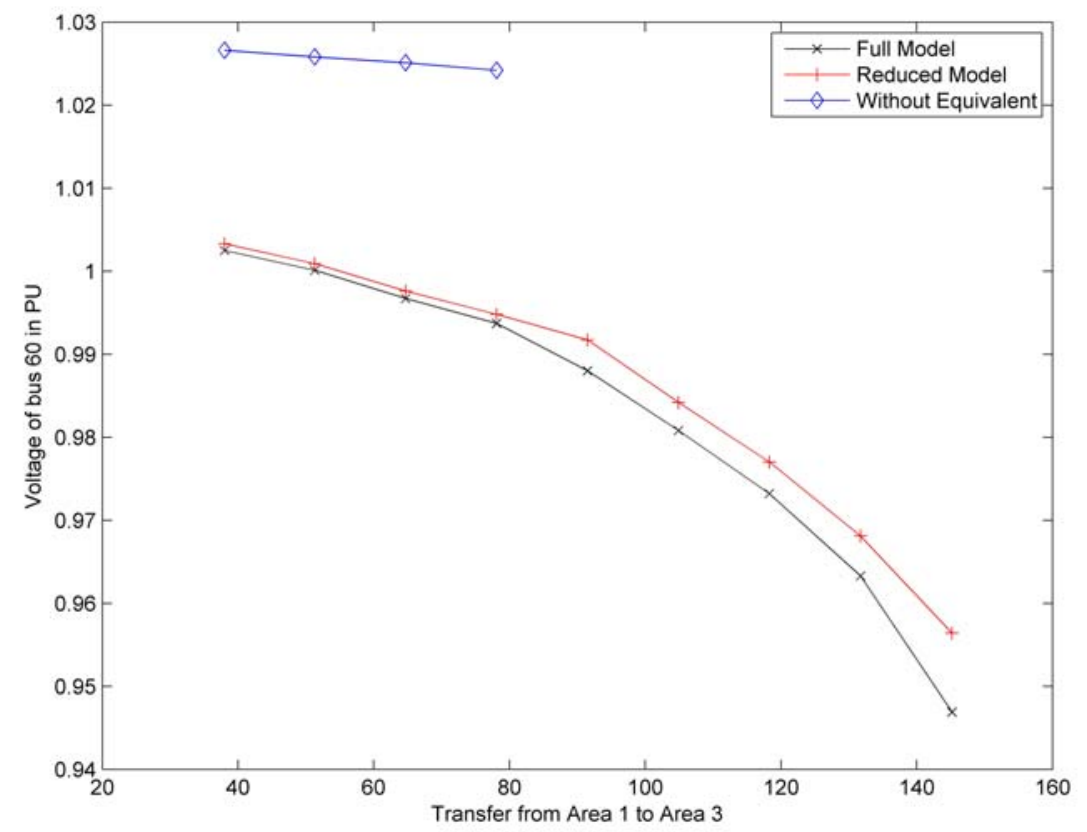

Figure 30: Post Contingency voltage variation of bus 60 with transfer level 


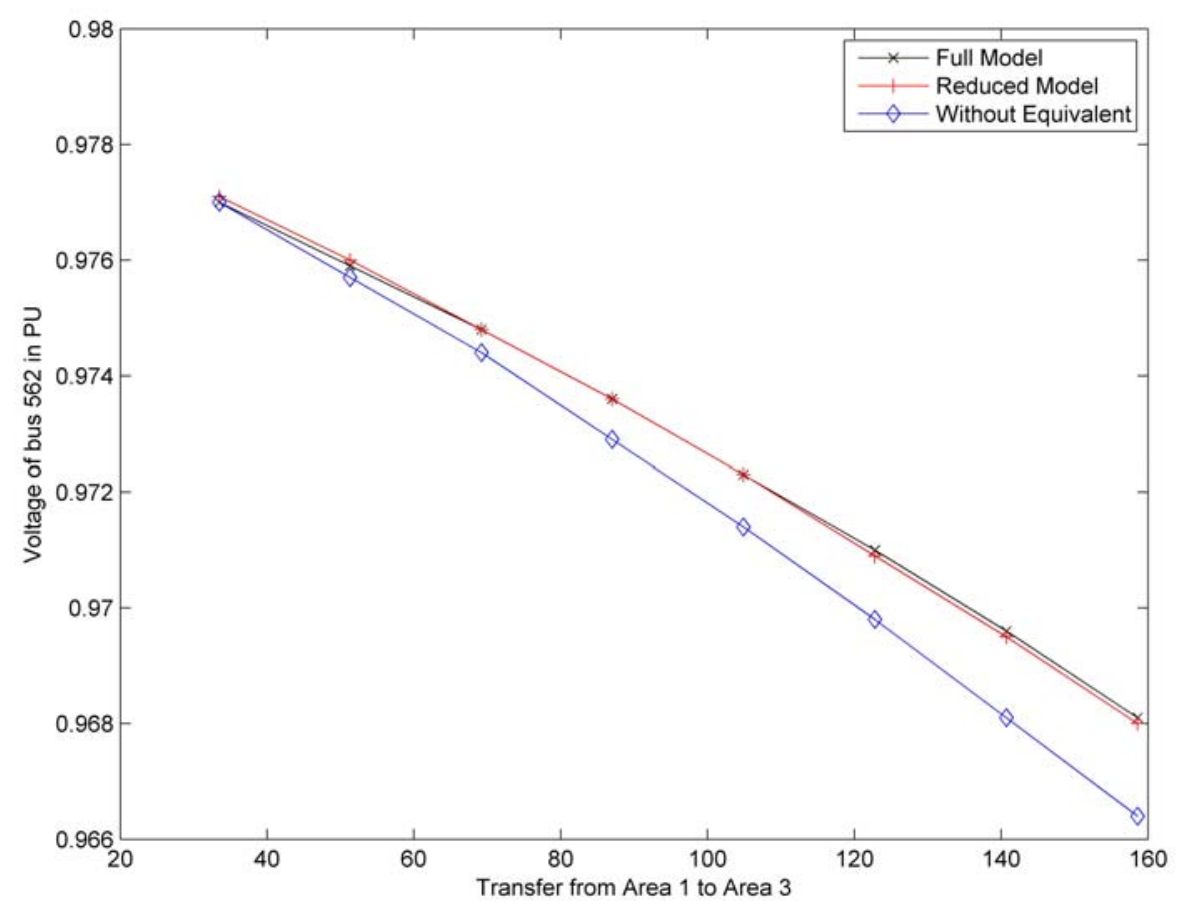

Figure 31: Pre Contingency voltage variation of bus 562 with transfer level

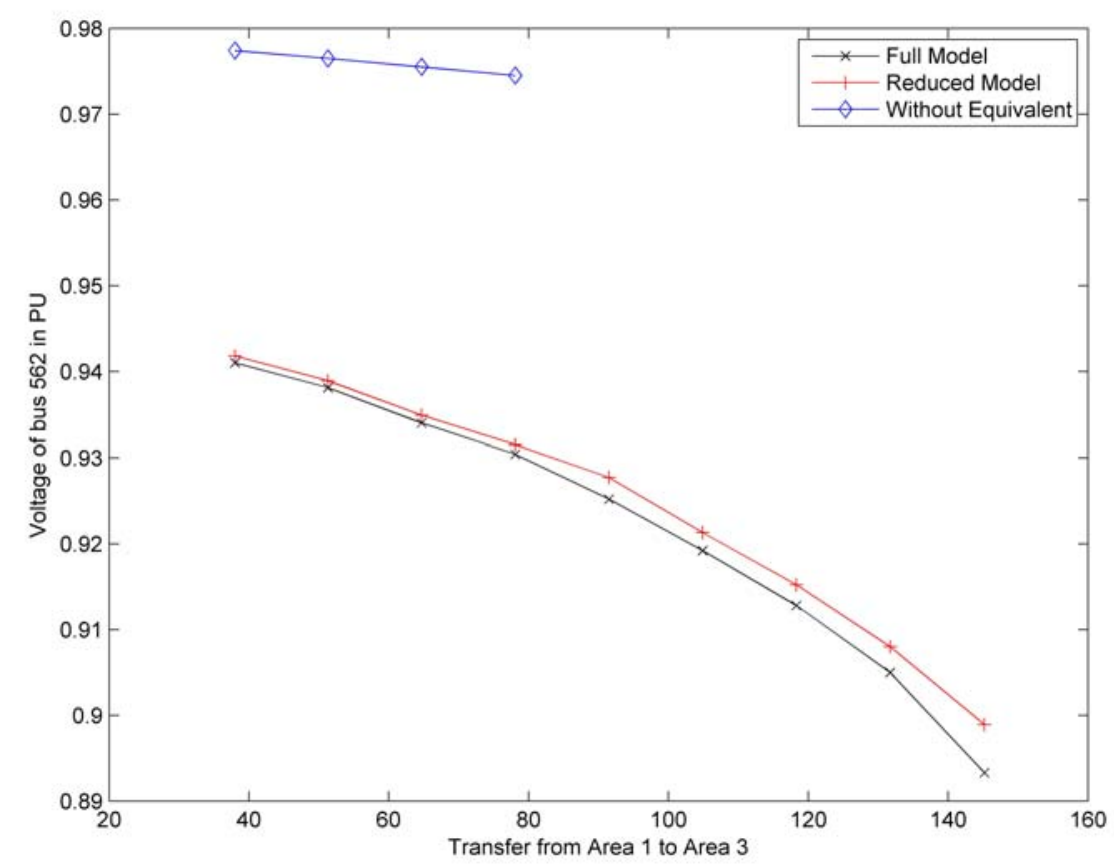

Figure 32: Post Contingency voltage variation of bus 562 with transfer level 
It can be seen that the pre and post contingency voltage variation of all the selected buses have shown very similar behaviour between the full model and the reduced model.

\subsection{Summary}

This chapter presented another method to develop an equivalent circuit using available boundary information. Modified IEEE three area 300 bus system was used to demonstrate the equivalent circuit creation process and model validation.

The validity of the equivalent circuit was thoroughly evaluated by performing contingency analysis, transfer analysis and PV analysis. All these analysis have shown that developed equivalent circuit behaves very similar to the full model and hence suitable for the real time security assessment. 


\section{Chapter 5}

\section{Model Validation with Manitoba $\mathrm{Hy}^{-}$}

\section{dro System}

This chapter provides an introduction to the Manitoba Hydro power system and demonstrates the need to develop an equivalent circuit for Manitoba Hydro. The second part of this chapter presents the equivalent circuit developed for Manitoba Hydro and model validation.

\subsection{Introduction to Manitoba Hydro}

Manitoba is a province located in central Canada between the Canadian provinces of Saskatchewan and Ontario, and north of the American states of North Dakota and Minnesota (as shown in Figure 33). Manitoba Hydro is the provincial utility responsible for generation, transmission, and distribution of electricity in the province of Manitoba. The 
utility supplies power to over a half million electricity customers spread over 650,000 square kilometers. Manitoba Hydro owns 17 generation stations, over $13000 \mathrm{~km}$ of transmission lines and $76000 \mathrm{~km}$ of distribution lines. The Manitoba Hydro system peak load for the 2014/15 period was 4773 MW [154].

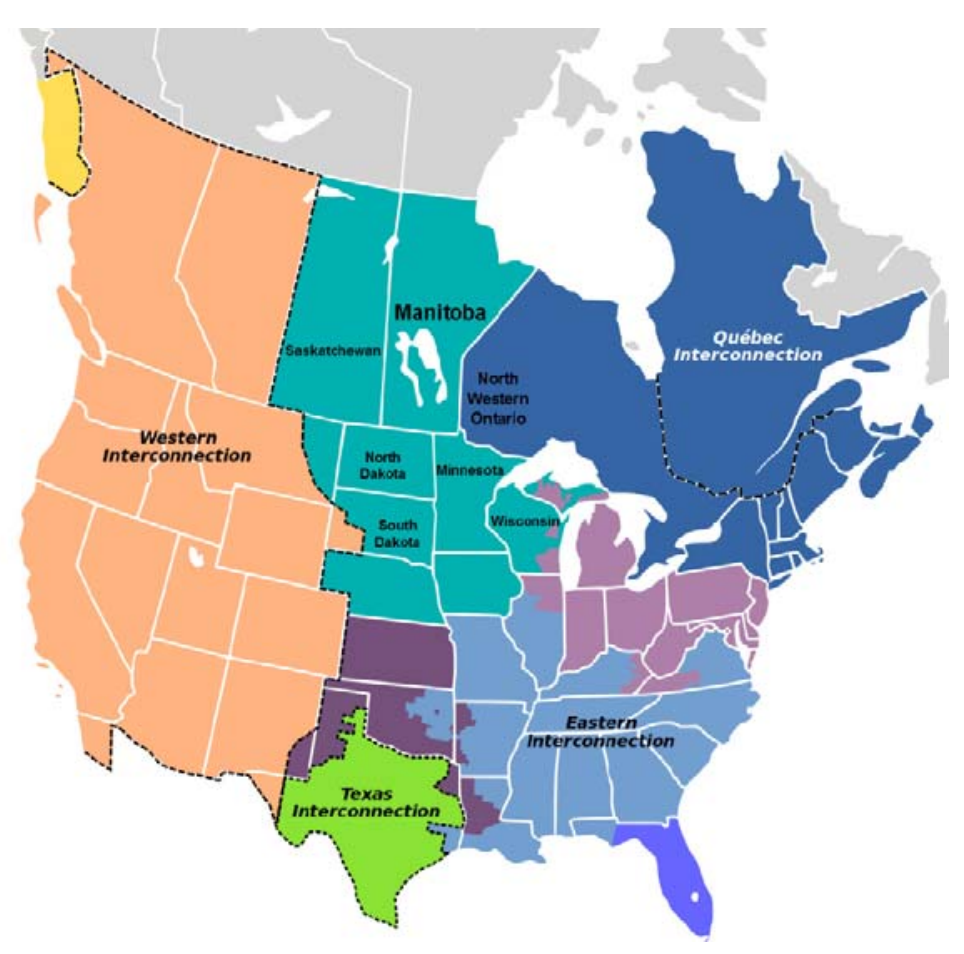

Figure 33: Geographical Location of Manitoba

\subsection{Manitoba Hydro Power System Model}

The basic layout of Manitoba Hydro's power system is shown in Figure 34. The majority of Manitoba Hydro generating stations are located in the northern part of the province. The three largest generating stations (Kettle, Long Spruce, and Limestone) are responsible for about $70 \%$ of the total generation. These three generating stations form an iso- 
lated, asynchronous island. Power from these generating stations is transmitted to Dorsey converter station (located close to Winnipeg, main load centre) through two HVDC bipoles.

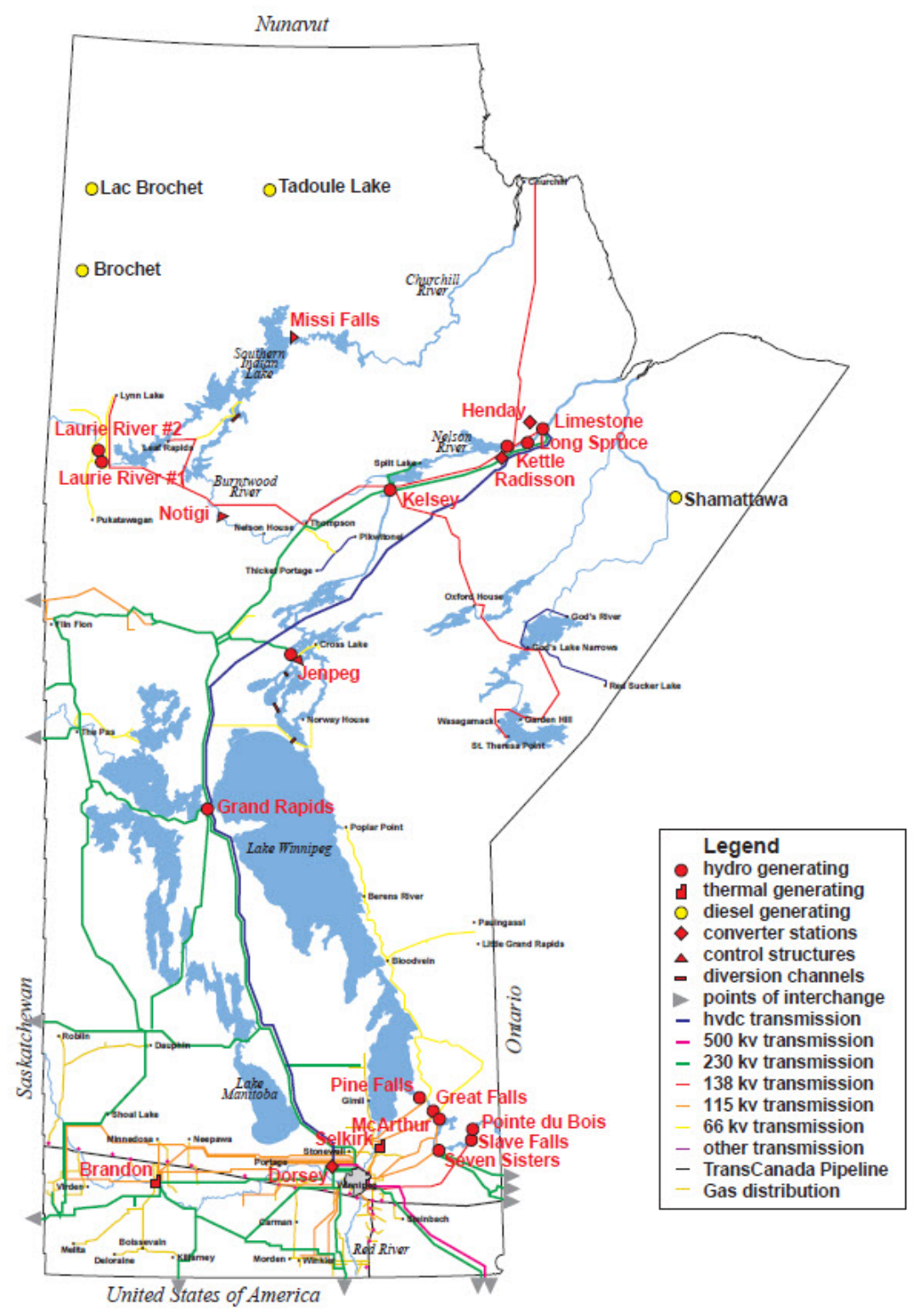

Figure 34: Manitoba Hydro Power system 
Manitoba's electrical system is connected to the Canadian provinces of Saskatchewan via three $230 \mathrm{kV}$ tie lines and connected to North Western Ontario via two $230 \mathrm{kV}$ lines with phase shifting transformers. It is connected to the United States of America via three $230 \mathrm{kV}$ tie lines and one $500 \mathrm{kV}$ tie line. The Manitoba Hydro EMS model contains the bulk electric system of Manitoba, and most major facilities in Saskatchewan, North Western Ontario, North Dakota, South Dakota, Minnesota, and parts of Montana and Wisconsin.

Flow into the boundary area from the cut-off area is modeled as loads at the boundary stations. The process is illustrated in Figure 35. Assuming arc AB is the EMS boundary, the EMS model contains all information left of arc AB. In other words, Station $\mathrm{Y}$ is the boundary station and Station $\mathrm{Z}$ is the cut off station. When developing the EMS model, flow coming into Station $\mathrm{Y}$ from Station $\mathrm{Z}$ is modeled as a load at Station Y. In real-time, the load connected to the power flow model is updated with the flow on the cut-off line and can be positive or negative based on the flow direction. 

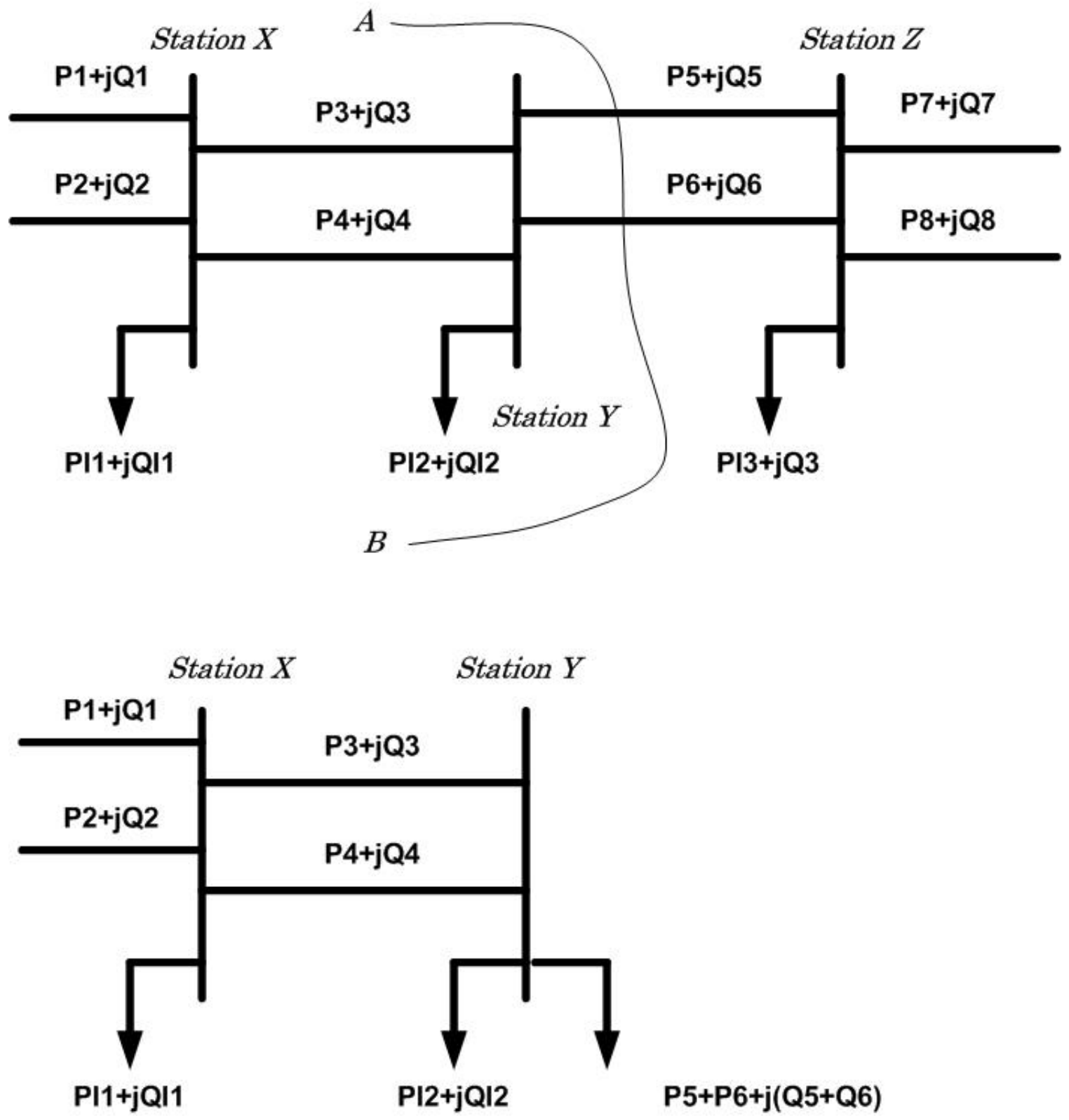

Figure 35: Modelling boundary buses in the EMS model. Actual system (above) and how it is modelled (below)

The Province of Saskatchewan is the Canadian boundary for the Eastern Interconnection. Saskatchewan is connected to the province of Alberta via a dc line and to the USA via a $230 \mathrm{kV}$ line with a phase shifting transformer. The Manitoba Hydro EMS 
boundary contains American facilities up to the Eastern Interconnection boundary in the states of South Dakota and Montana. Only a few dc lines exist between the Eastern Interconnection and the Western Interconnection. Therefore, Manitoba Hydro's EMS model contains sufficient modelling information west of the province.

Ontario's power system and its various interconnections are shown in Figure 36. North western Ontario system (shown in the top right hand of the Figure 36) is connected to the rest of the Ontario bulk electric system via two $230 \mathrm{kV}$ tie lines, called the EastWest ties and one $115 \mathrm{kV}$ tie line with a phase shifting transformer to the American state of Minnesota. Ontario system is also connected to the united state of America across three other interfaces. Ontario system also connected to the Quebec interconnection. However, all the interconnections between Ontario and Quebec are dc ties.

Close examination of Figure 36 and Figure 33 shows that there are three parallel paths existing between Manitoba and North western Ontario. The first path is across the phase shifters at the Manitoba - Ontario border near Kenora, a second path is across the phase shifter at the USA-Ontario border near International Falls, Minnesota, and a third path is through the East-West ties into the Ontario system and through the USA. Manitoba Hydro's EMS model contains the first two paths. However, due to the large geographical area it is not possible to include the third path in Manitoba Hydro's EMS model. 


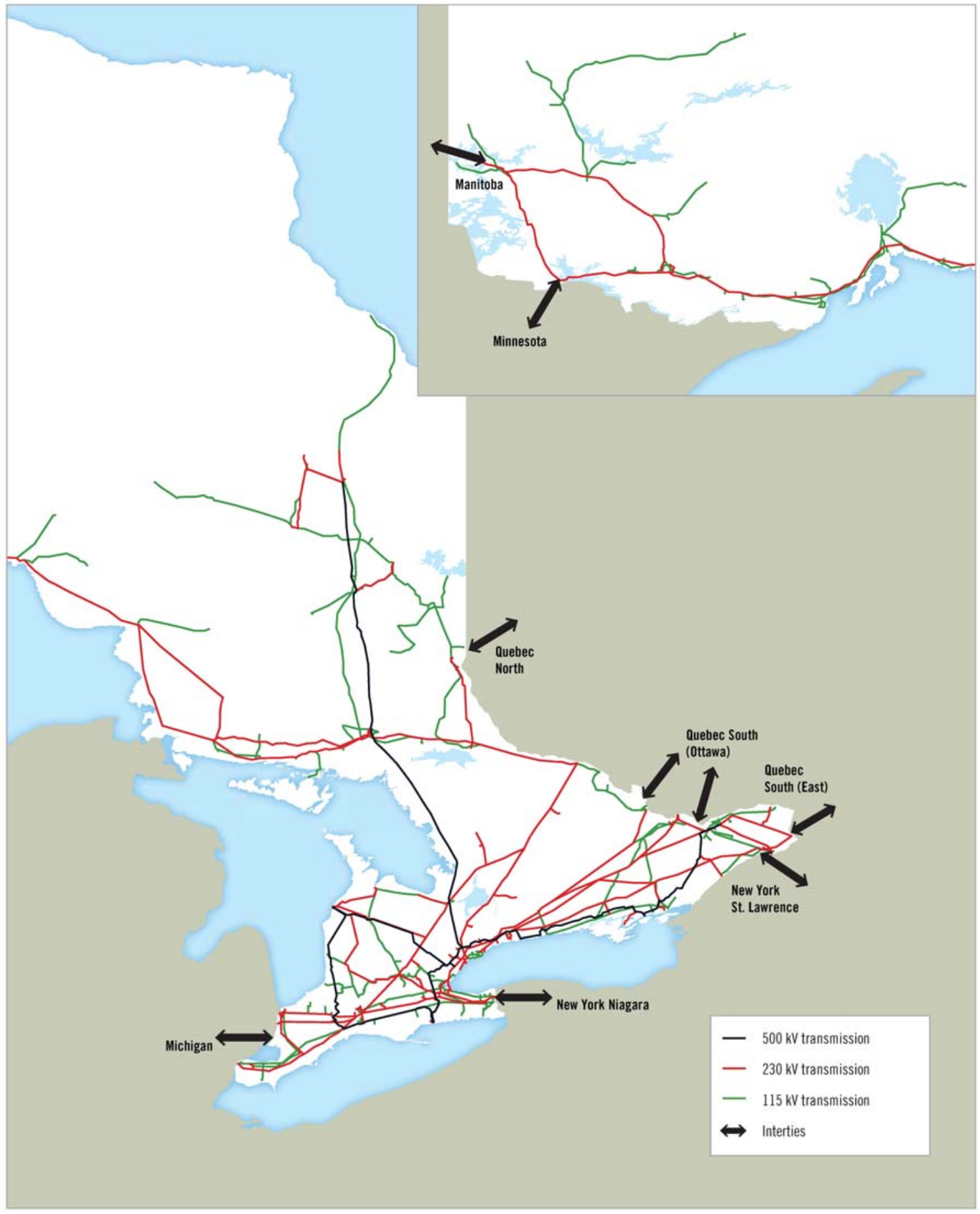

Figure 36: Ontario Interconnections 
Figure 37 and Figure 38 show the flow on Manitoba Hydro's $500 \mathrm{kV}$ line and the Ontario East-West line just before and after a trip of the $500 \mathrm{kV}$ tie line between Manitoba Hydro and USA. These data were obtained from Manitoba Hydro's real-time data archive. It can be seen that the East-West tie flow changes by approximately $80 \mathrm{MW}$ when the $500 \mathrm{kV}$ tie line tripped. However, Manitoba Hydro EMS model is cut off at North western Ontario and East-West tie lines are modelled as load at the boundary station as shown in Figure 35. Manitoba Hydro's current EMS model cannot demonstrate this behavior today. As East-West tie lines are modelled as load at the boundary, if the same contingency is simulated in the EMS model, flow change cannot be observed. Therefore, there is a need to develop an equivalent circuit to capture the impact of this high impedance path in the EMS models.

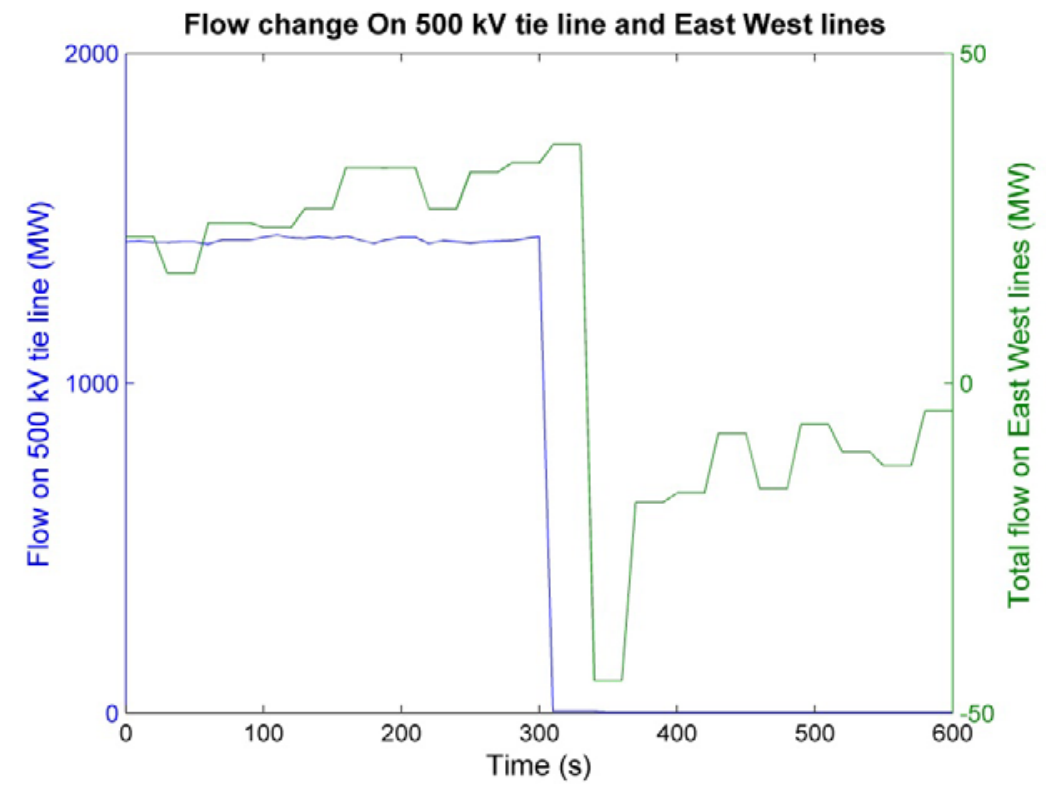

Figure 37: Flow Change on $500 \mathrm{kV}$ line and East-West tie lines 


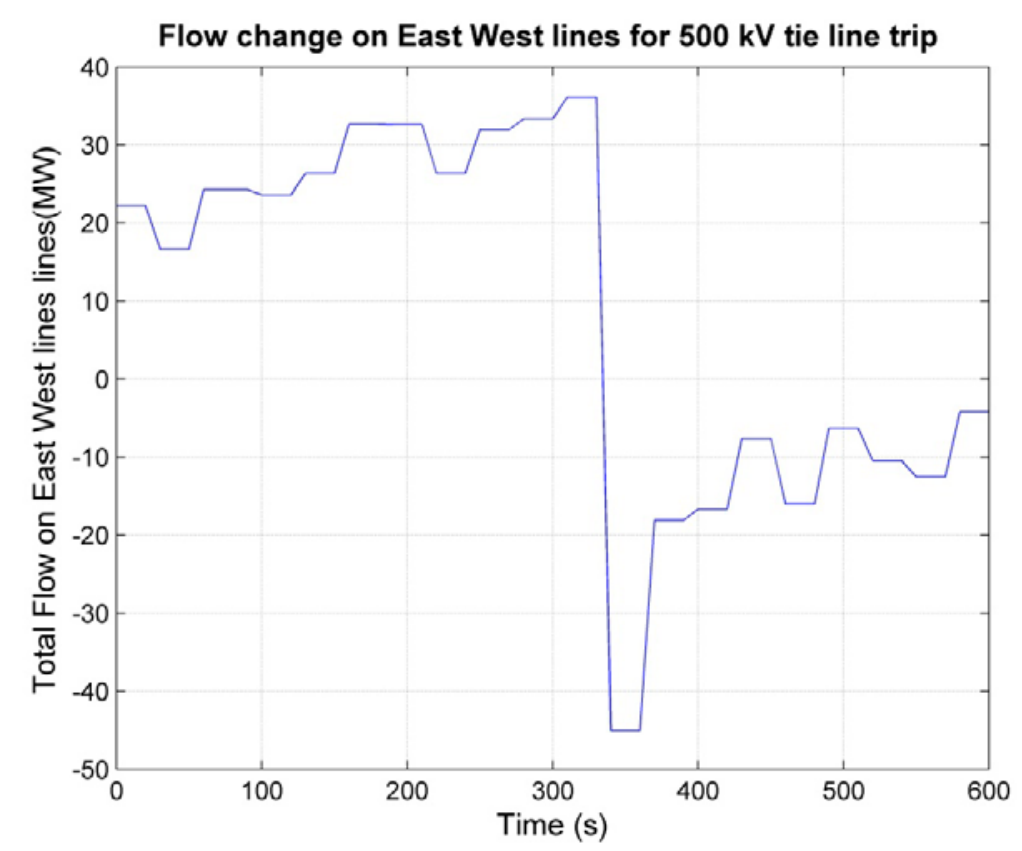

Figure 38: Flow change on East-West tie line for Loss of $500 \mathrm{kV}$ Line in Manitoba

\subsection{Creating Equivalent Circuit for Manitoba}

\section{Hydro System}

The proposed method described in Chapter 3 was used to develop an equivalent circuit between the USA and North western Ontario. A power transfer scenario was created between Manitoba and Ontario using the off-line study model, which is used in Manitoba Hydro system studies, to calculate the equivalent impedance and select the boundary stations. This exercise identified 8 boundary stations in the USA which can be connected to North western Ontario. Locations of these stations are shown in Figure 39. These stations include five $345 \mathrm{kV}$ stations along the boundary (Raun, Fort Thompson, Rockey Run, 
Adams, Gardner Park), two $230 \mathrm{kV}$ stations along the boundary (Fort Randall and New Underwood), and one $115 \mathrm{kV}$ station (Alma). The equivalent circuit parameters obtained following this method are shown in table 1 .

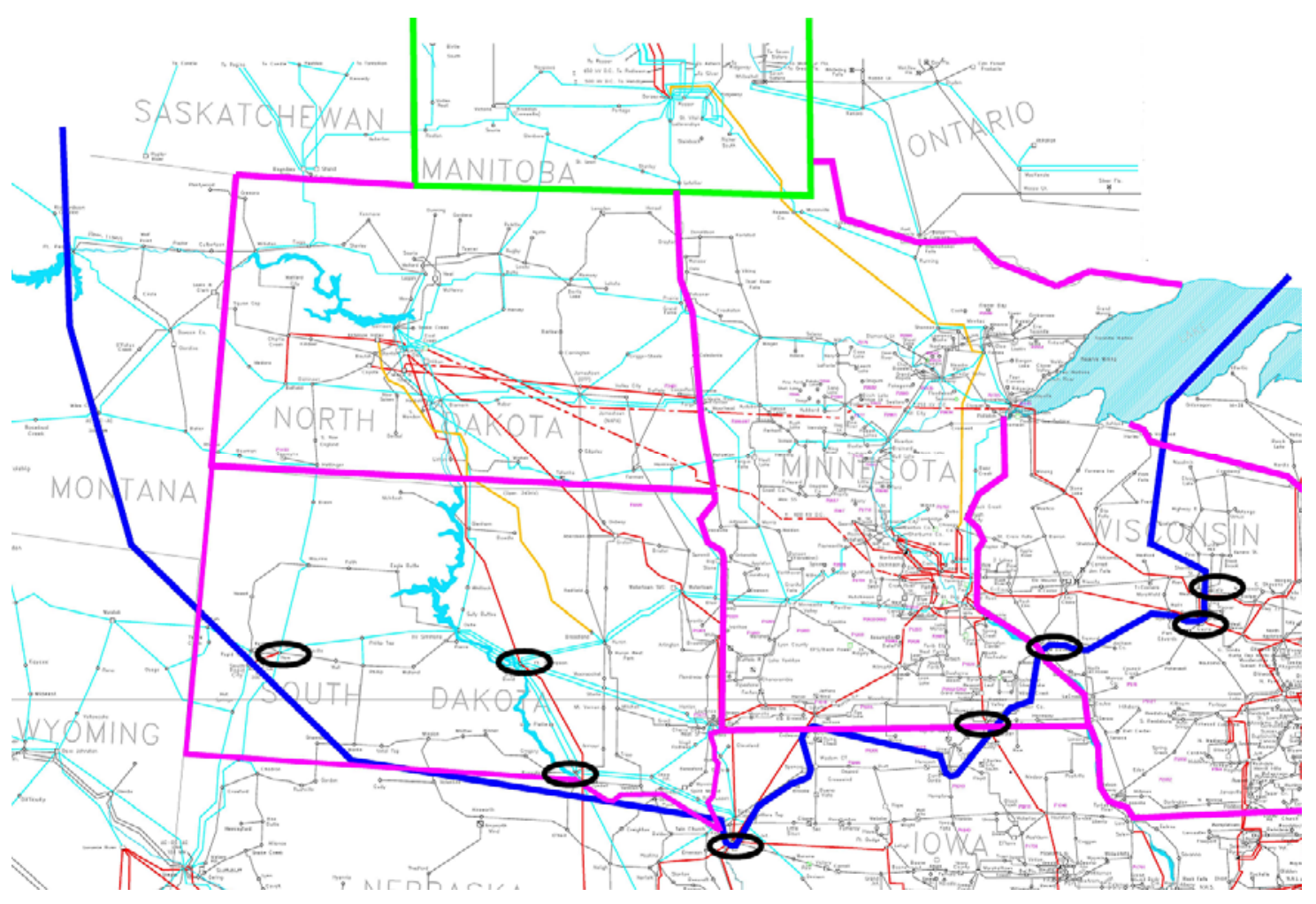

Figure 39: Location of Selected Boundary Stations in the USA 
Table 13: Equivalent Circuit parameters

\begin{tabular}{|l|l|}
\hline Link & Equivalent Circuit reactance (in pu) \\
\hline Wawa to Raun & 1.22 \\
\hline Wawa to Fort Thompson & 3.58 \\
\hline Wawa to Rockey Run & 1.92 \\
\hline Wawa to Adams & 1.11 \\
\hline Wawa to Gardener Park & 2.78 \\
\hline Wawa to New Under Wood & 8.60 \\
\hline Wawa to Fort Randall & 7.29 \\
\hline Wawa to Alma & 7.07 \\
\hline
\end{tabular}

Once the equivalent circuit parameters were calculated, the equivalent circuit was created from the off-line study model by removing lines along the Manitoba Hydro EMS boundary. A total of 91 lines were disconnected in this exercise. Power flows coming from the external area into the internal area on those boundary lines (except at the identified equivalent location points) were modeled as loads. For example, if a particular boundary station had $20 \mathrm{MW}$ and 7 MVAr of power flowing into the external area through a cut-off line, this was modeled as a load with active power of $20 \mathrm{MW}$ and 7 MVAr at the boundary stations. After all loads were modeled at the boundary stations, the equivalent circuit was added to the model to connect North Western Ontario with the identified boundary stations in the USA. Once the equivalent circuit was connected, the boundary matching exercise explained in Chapter 3 was carried out so that the internal lines in the reduced model would have the same power flow as the full model. 


\subsection{Benchmarking the Equivalent Circuit}

Various types of benchmarking exercises were carried out to evaluate the accuracy of the equivalent circuit. The benchmarking methods included contingency analysis, transfer calculation, and PV analysis. The following section describes the results of the benchmarking exercises.

\subsubsection{Contingency Analysis}

The accuracy of the equivalent model was first evaluated by comparing the contingency analysis using the full off-line study model and the reduced model derived with the proposed methodology. The results of the contingency analysis which compare following parameters between the two models are provided in Table 15 to Table 20:

- Voltage of key Manitoba Stations

- Active power flow along key interfaces

- Reactive power flow along key interfaces

- Active power along key tie lines

- Reactive power along key tie lines

- Phase shifter angles of phase shifters located close to Manitoba

The six most critical contingencies were selected for the analysis and include the four tie lines between Manitoba and the USA (lines 1 to 4), the tie line between Saskatchewan and the USA (Line 5) and the tie line between North Western Ontario and the USA (Line 
6). The pre-contingency flows on the lines are provided in Table 14 and the geographic locations of the lines are shown in Figure 40.

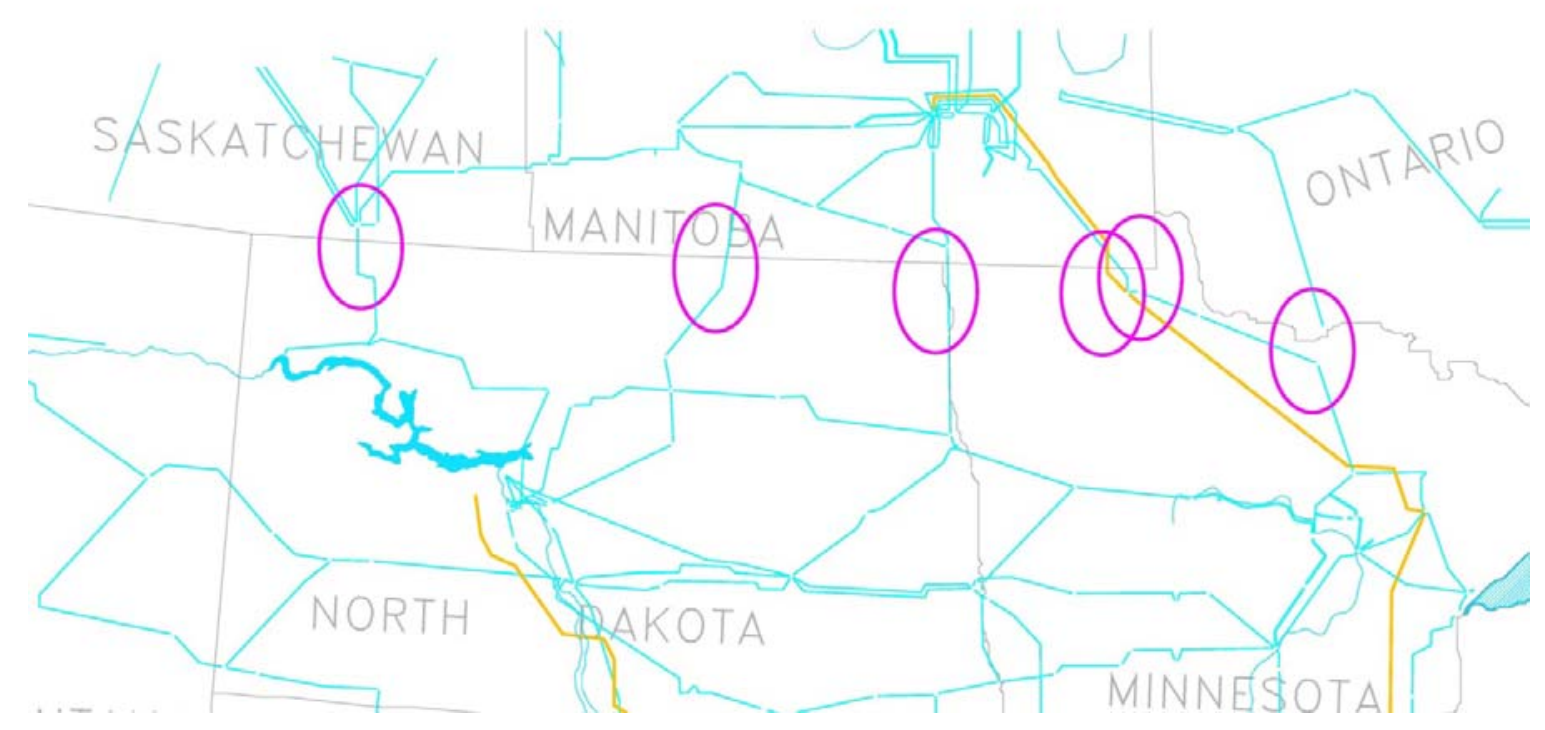

Figure 40: Lines Selected for Contingency Analysis

Table 14: Pre Contingency Flow on Selected Lines

\begin{tabular}{|l|l|}
\hline Line & Pre contingency MW flow \\
\hline Line 1 & 54 \\
\hline Line 2 & 270 \\
\hline Line 3 & 1102 \\
\hline Line 4 & 78 \\
\hline Line 5 & 166 \\
\hline Line 6 & 152 \\
\hline
\end{tabular}


The six lines were selected for the contingency analysis due to the following reasons:

- These lines provide direct and indirect connection between Manitoba and the USA. Therefore, any inaccuracies in the equivalent circuit should be visible in these contingency analyses.

- The four tie lines between Manitoba and the USA have Special Protection Schemes (SPS's) to reduce Manitoba Hydro DC by the pre-contingency flow on the line. These SPS schemes were modelled in the simulation. Therefore, these contingencies behave like generation losses. For example, loss of Line 3 will result in reducing Manitoba Hydro DC by 1102 MW.

- Line 6 connects North Western Ontario with the USA. A fraction of the power redirects through Manitoba when this line is lost and the rest goes to the main Ontario system. As mentioned before, Manitoba Hydro's system does not contain the main Ontario system; it is cut off at North Western Ontario. Equivalent impedances should be accurate in order to simulate this contingency accurately.

The first quantity compared was the bus voltages in the various Manitoba stations. Five stations were selected for this analysis, which include the Manitoba Hydro terminal station on the Manitoba - Ontario interface (Station 1) and four terminal stations on the Manitoba - USA interface (Station 2 to Station 5). Voltage comparisons between the two models are shown in Table 15. The highest error reported for the voltage was 0.0008 p.u. or the $0.08 \%$, demonstrating the equivalent circuit can produce accurate voltage profiles in Manitoba for the contingency analysis. 
Table 15: Voltage Comparison (in p.u.)

\begin{tabular}{|l|c|c|c|c|c|}
\hline & $\begin{array}{c}\text { Station } \\
\mathbf{1}\end{array}$ & $\begin{array}{c}\text { Station } \\
\mathbf{2}\end{array}$ & $\begin{array}{c}\text { Station } \\
\mathbf{3}\end{array}$ & $\begin{array}{c}\text { Station } \\
\mathbf{4}\end{array}$ & $\begin{array}{c}\text { Station } \\
\mathbf{5}\end{array}$ \\
\hline Pre contingency & 1.0811 & 1.0137 & 1.0234 & 1.0450 & 1.0231 \\
\hline Full & 1.0812 & 1.0135 & 1.0232 & 1.0450 & 1.0230 \\
\hline Reduced & -0.0001 & 0.0002 & 0.0002 & 0.0000 & 0.0001 \\
\hline Difference
\end{tabular}

Contingency of Line 1

\begin{tabular}{|l|l|l|l|l|l|}
\hline Full & 1.0812 & 1.0149 & 1.0236 & 1.0450 & 1.0236 \\
\hline Reduced & 1.0812 & 1.0145 & 1.0234 & 1.0450 & 1.0235 \\
\hline Difference & 0.0000 & 0.0004 & 0.0002 & 0.0000 & 0.0001 \\
\hline
\end{tabular}

Contingency of Line 2

\begin{tabular}{|l|l|l|l|l|l|}
\hline Full & 1.0812 & 1.0137 & 1.0333 & 1.0450 & 1.0234 \\
\hline Reduced & 1.0812 & 1.0130 & 1.0331 & 1.0450 & 1.0233 \\
\hline Difference & 0.0000 & 0.0007 & 0.0002 & 0.0000 & 0.0001 \\
\hline
\end{tabular}

Contingency of Line 3

\begin{tabular}{|l|l|l|l|l|l|}
\hline Full & 1.0811 & 1.0140 & 1.0232 & 1.0450 & 1.0187 \\
\hline Reduced & 1.0811 & 1.0139 & 1.0231 & 1.0450 & 1.0187 \\
\hline Difference & 0.0000 & 0.0001 & 0.0001 & 0.0000 & 0.0000 \\
\hline
\end{tabular}

Contingency of Line 4

\begin{tabular}{|l|r|r|r|r|r|}
\hline Full & 1.0811 & 1.0138 & 1.0236 & 1.0450 & 1.0328 \\
\hline Reduced & 1.0812 & 1.0135 & 1.0234 & 1.0450 & 1.0328 \\
\hline Difference & -0.0001 & 0.0003 & 0.0002 & 0.0000 & 0.0000 \\
\hline
\end{tabular}

Contingency of Line 5

\begin{tabular}{|l|l|l|l|l|l|}
\hline Full & 1.0811 & 0.9999 & 1.0163 & 1.0450 & 1.0211 \\
\hline Reduced & 1.0811 & 0.9991 & 1.0158 & 1.0450 & 1.0210 \\
\hline Difference & 0.0000 & 0.0008 & 0.0005 & 0.0000 & 0.0001 \\
\hline
\end{tabular}

Contingency of Line 6

\begin{tabular}{|l|r|r|r|r|r|}
\hline Full & 1.0819 & 1.0135 & 1.0231 & 1.0450 & 1.0191 \\
\hline Reduced & 1.0820 & 1.0130 & 1.0229 & 1.0450 & 1.0189 \\
\hline Difference & -0.0001 & 0.0005 & 0.0002 & 0.0000 & 0.0002 \\
\hline
\end{tabular}


The second quantity compared was the active power flow across the various interfaces. Nine interfaces were selected for this analysis which, include:

- Manitoba - USA interface (USA), Manitoba-Ontario interface (ON),

- Manitoba - Saskatchewan interface (SPC),

- North Western Ontario at the boundary cut-off point (E-W ties),

- $345 \mathrm{kV}$ Raun boundary station located in the USA (Raun),

- $345 \mathrm{kV}$ Adams boundary station located in the USA (Adams),

- $345 \mathrm{kV}$ Rockey Run boundary station located in the USA (Rockey Run),

- $345 \mathrm{kV}$ Garden Park boundary station located in the USA (Garden Park), and

- $345 \mathrm{kV}$ Forth Thompson boundary station located in the USA (Ft. Thompson).

The results of the analysis are shown in Table 16. The highest error was observed for the USA and ON interfaces for the contingent loss of Line 6 which is the loss of the tie line between North western Ontario and the USA. 
Table 16: Interface Active Power Comparison (MW)

\begin{tabular}{|c|c|c|c|c|c|c|c|c|c|}
\hline & USA & ON & SPC & $\begin{array}{c}\text { E-W } \\
\text { ties }\end{array}$ & Raun & Adams & $\begin{array}{l}\text { Rocky } \\
\text { Run }\end{array}$ & $\begin{array}{c}\text { Garden } \\
\text { Park } \\
\end{array}$ & $\begin{array}{c}\text { Ft. } \\
\text { Thompson }\end{array}$ \\
\hline \multicolumn{10}{|c|}{ Pre contingency } \\
\hline Full & 1504.7 & 19 & 4.3 & 191.8 & .7 & 8.7 & 8.6 & & -65.8 \\
\hline & 1506.8 & 197.9 & 63.6 & 194.3 & 436.6 & 9.6 & 7.5 & -466.7 & -67.2 \\
\hline Difference & -2.1 & 1.4 & 0.7 & -2.5 & 1.1 & 0.9 & -1.1 & -5.9 & 1.4 \\
\hline
\end{tabular}

Contingency of Line 1

\begin{tabular}{|l|r|r|r|r|r|r|r|r|r|}
\hline Full & 1428.9 & 197.5 & 67.6 & 193.3 & 437.4 & -183.5 & -530.1 & -472.9 & -60.7 \\
\hline Reduced & 1428.9 & 196.2 & 67.4 & 195.9 & 436.5 & -181.0 & -527.9 & -466.7 & -62.9 \\
\hline Difference & 0.0 & 1.3 & 0.2 & -2.6 & 0.9 & -2.5 & -2.2 & -6.2 & 2.2 \\
\hline
\end{tabular}

Contingency of Line 2

\begin{tabular}{|l|r|r|r|r|r|r|r|r|r|}
\hline Full & 1241.0 & 198.2 & 67.4 & 194.1 & 439.5 & -195.5 & -533.2 & -472.7 & -50.8 \\
\hline Reduced & 1240.8 & 197.2 & 67.3 & 196.2 & 437.4 & -184.1 & -528.7 & -466.1 & -55.3 \\
\hline Difference & 0.2 & 1.0 & 0.1 & -2.1 & 2.1 & -11.4 & -4.5 & -6.6 & 4.5 \\
\hline
\end{tabular}

Contingency of Line 3

\begin{tabular}{|l|r|r|r|r|r|r|r|r|r|}
\hline Full & 409.6 & 199.6 & 59.2 & 199.2 & 425.8 & -197.6 & -520.3 & -456.0 & -59.5 \\
\hline Reduced & 411.5 & 198.4 & 58.3 & 201.3 & 433.8 & -185.1 & -528.8 & -461.6 & -60.8 \\
\hline Difference & -1.9 & 1.2 & 0.9 & -2.1 & -8.0 & -12.5 & 8.5 & 5.6 & 1.3 \\
\hline
\end{tabular}

Contingency of Line 4

\begin{tabular}{|l|r|r|r|r|r|r|r|r|r|}
\hline Full & 1426.9 & 201.1 & 63.8 & 200.5 & 435.1 & -183.4 & -530.0 & -472.4 & -65.1 \\
\hline Reduced & 1428.6 & 200.1 & 63.2 & 199.9 & 435.2 & -181.5 & -528.5 & -466.7 & -66.5 \\
\hline Difference & -1.7 & 1.0 & 0.6 & 0.6 & -0.1 & -1.9 & -1.5 & -5.7 & 1.4 \\
\hline
\end{tabular}

Contingency of Line 5

\begin{tabular}{|l|r|r|r|r|r|r|r|r|r|}
\hline Full & 1648.6 & 200.9 & -88.6 & 187.8 & 440.3 & -187.9 & -532.9 & -475.6 & -47.9 \\
\hline Reduced & 1647.9 & 201.1 & -88.5 & 189.8 & 438.8 & -181.1 & -527.5 & -466.8 & -52.2 \\
\hline Difference & 0.7 & -0.2 & -0.1 & -2.0 & 1.5 & -6.8 & -5.4 & -8.8 & 4.3 \\
\hline
\end{tabular}

Contingency of Line 6

\begin{tabular}{|l|r|r|r|r|r|r|r|r|r|}
\hline Full & 1570.5 & 132.4 & 63.3 & 96.8 & 452.7 & -157.9 & -513.3 & -461.9 & -62.4 \\
\hline Reduced & 1578.3 & 124.7 & 62.7 & 104.5 & 459.5 & -157.8 & -514.7 & -457.9 & -63.2 \\
\hline Difference & -7.8 & 7.7 & 0.6 & -7.7 & -6.8 & -0.1 & 1.4 & -4.0 & 0.8 \\
\hline
\end{tabular}


The third quantity compared was the interface MVAr flow for the same nine interfaces. The results are shown in Table 17. It can be seen that reactive power flow comparison between most of the interfaces produced very satisfactory results. The largest error was 13.2 MVAr and was observed for total reactive power coming in from Rocky Run station (RockyRun) for the loss of line 6. Of the 13.2 MVAr, 7.7 MVAr is related to the errors in the steady state power flow. 
Table 17: Interface Reactive Power Comparison (MVAr)

\begin{tabular}{|c|c|c|c|c|c|c|c|c|c|}
\hline & USA & ON & SPC & $\begin{array}{c}\text { E-W } \\
\text { ties }\end{array}$ & Raun & Adams & $\begin{array}{c}\text { Rocky } \\
\text { Run }\end{array}$ & $\begin{array}{c}\text { Garden } \\
\text { Park }\end{array}$ & $\begin{array}{c}\text { Ft. } \\
\text { Thompson }\end{array}$ \\
\hline \multicolumn{10}{|c|}{ Pre contingency } \\
\hline Full & -95.7 & -86.1 & -53.0 & -45.8 & -63.2 & -37.2 & -20.3 & 16.5 & 8.2 \\
\hline Reduced & -95.5 & -85.8 & -52.9 & -46.0 & -63.7 & -36.3 & -28.0 & 21.6 & 8.6 \\
\hline Difference & -0.2 & -0.3 & -0.1 & 0.2 & 0.5 & -0.9 & 7.7 & -5.1 & -0.4 \\
\hline
\end{tabular}

Contingency of Line 1

\begin{tabular}{|l|r|r|r|r|r|r|r|r|r|}
\hline Full & -90.6 & -86.3 & -52.5 & -45.7 & -64.0 & -36.4 & -19.7 & 16.3 & 7.5 \\
\hline Reduced & -90.6 & -86.1 & -52.6 & -45.8 & -64.8 & -36.0 & -27.8 & 21.8 & 8.0 \\
\hline Difference & 0.0 & -0.2 & 0.1 & 0.1 & 0.8 & -0.4 & 8.1 & -5.5 & -0.5 \\
\hline
\end{tabular}

\section{Contingency of Line 2}

\begin{tabular}{|l|r|r|r|r|r|r|r|r|r|}
\hline Full & -73.6 & -85.9 & -53.1 & -45.8 & -65.6 & -34.2 & -19.1 & 16.1 & 6.2 \\
\hline Reduced & -74.6 & -85.6 & -53.2 & -45.7 & -66.9 & -35.5 & -27.5 & 21.8 & 6.9 \\
\hline Difference & 1.0 & -0.3 & 0.1 & -0.1 & 1.3 & 1.3 & 8.4 & -5.7 & -0.7 \\
\hline
\end{tabular}

\section{Contingency of Line 3}

\begin{tabular}{|l|r|r|r|r|r|r|r|r|r|}
\hline Full & -33.3 & -83.4 & -52.0 & -44.9 & -62.5 & -33.7 & -18.7 & 17.7 & 7.3 \\
\hline Reduced & -33.3 & -83.3 & -51.9 & -45.8 & -64.4 & -35.2 & -27.4 & 21.3 & 7.6 \\
\hline Difference & 0.0 & -0.1 & -0.1 & 0.9 & 1.9 & 1.5 & 8.7 & -3.6 & -0.3 \\
\hline
\end{tabular}

\section{Contingency of Line 4}

\begin{tabular}{|l|r|r|r|r|r|r|r|r|r|}
\hline Full & -97.7 & -85.7 & -52.8 & -45.2 & -63.4 & -36.4 & -19.7 & 16.7 & 8.1 \\
\hline Reduced & -98.0 & -86.2 & -52.9 & -46.2 & -63.9 & -36.1 & -27.7 & 21.7 & 8.5 \\
\hline Difference & 0.3 & 0.5 & 0.1 & 1.0 & 0.5 & -0.3 & 8.0 & -5.0 & -0.4 \\
\hline
\end{tabular}

\section{Contingency of Line 5}

\begin{tabular}{|l|r|r|r|r|r|r|r|r|r|}
\hline Full & -79.4 & -86.1 & -28.0 & -46.0 & -65.5 & -35.5 & -19.5 & 15.9 & 5.7 \\
\hline Reduced & -80.4 & -85.6 & -28.3 & -44.1 & -66.7 & -35.8 & -27.7 & 21.8 & 6.4 \\
\hline Difference & 1.0 & -0.5 & 0.3 & -1.9 & 1.2 & 0.3 & 8.2 & -5.9 & -0.7 \\
\hline
\end{tabular}

\section{Contingency of Line 6}

\begin{tabular}{|l|r|r|r|r|r|r|r|r|r|}
\hline Full & -96.7 & -88.2 & -52.9 & -45.7 & -63.7 & -40.8 & -21.7 & 17.4 & 7.8 \\
\hline Reduced & -95.5 & -88.2 & -53.1 & -46.8 & -63.9 & -40.1 & -34.9 & 17.0 & 8.2 \\
\hline Difference & -1.2 & 0.0 & 0.2 & 1.1 & 0.2 & -0.7 & 13.2 & 0.4 & -0.4 \\
\hline
\end{tabular}


The fourth variable compared was the active power flow along the same 6 lines used in the contingency analysis. As mentioned before these 6 lines consist of the 4 tie lines between Manitoba and USA (Line 1 to Line 4), the tie line between Saskatchewan and USA (Line 5), and the line between North Western Ontario and the USA (Line 6). The Special Protection Schemes (SPS's) associated with these lines, which reduce Manitoba Hydro DC by the pre-contingency flow on the lines, were simulated in the contingencies. Active power flow comparisons between the two scenarios are provided in Table 18. It can be seen that the largest error observed was 4.7 MW (percentage error of $0.42 \%$ ) on the Manitoba Hydro $500 \mathrm{kV}$ tie line for the loss of the tie line between North Western Ontario and the USA (Line 6). This result demonstrates the active power comparison produces very satisfactory results between the two models. 
Table 18: Active Power Comparison Along the Lines (MW)

\begin{tabular}{|c|c|c|c|c|c|c|}
\hline & Line 1 & Line 2 & Line 3 & Line 4 & Line 5 & Line 6 \\
\hline \multicolumn{7}{|c|}{ Pre contingency } \\
\hline Full & 54.0 & 270.3 & 1102.2 & 78.2 & 166.0 & 152.4 \\
\hline Reduced & 55.0 & 271.2 & 1102.6 & 78.0 & 165.3 & 153.5 \\
\hline Difference & -1.0 & -0.9 & -0.4 & 0.2 & 0.7 & -1.1 \\
\hline
\end{tabular}

Contingency of Line 1

\begin{tabular}{|l|r|r|r|r|r|r|}
\hline Full & 0.0 & 276.9 & 1075.9 & 76.1 & 169.5 & 152.1 \\
\hline Reduced & 0.0 & 278.3 & 1074.7 & 75.8 & 169.4 & 153.1 \\
\hline Difference & 0.0 & -1.4 & 1.2 & 0.3 & 0.1 & -1.0 \\
\hline
\end{tabular}

Contingency of Line 2

\begin{tabular}{|l|r|r|r|r|r|r|}
\hline Full & 94.8 & 0.0 & 1068.4 & 77.9 & 169.3 & 153.7 \\
\hline Reduced & 98.0 & 0.0 & 1065.1 & 77.8 & 169.1 & 154.5 \\
\hline Difference & -3.2 & 0.0 & 3.3 & 0.1 & 0.2 & -0.8 \\
\hline
\end{tabular}

Contingency of Line 3

\begin{tabular}{|l|r|r|r|r|r|r|}
\hline Full & 48.7 & 268.2 & 0.0 & 92.7 & 160.6 & 159.8 \\
\hline Reduced & 49.4 & 269.0 & 0.0 & 93.1 & 159.6 & 159.7 \\
\hline Difference & -0.7 & -0.8 & 0.0 & -0.4 & 1.0 & 0.1 \\
\hline
\end{tabular}

Contingency of Line 4

\begin{tabular}{|l|r|r|r|r|r|r|}
\hline Full & 53.4 & 270.4 & 1103.1 & 0.0 & 165.5 & 162.4 \\
\hline Reduced & 54.4 & 271.3 & 1103.0 & 0.0 & 164.7 & 160.6 \\
\hline Difference & -1.0 & -0.9 & 0.1 & 0.0 & 0.8 & 1.8 \\
\hline
\end{tabular}

Contingency of Line 5

\begin{tabular}{|l|r|r|r|r|r|r|}
\hline Full & 90.8 & 299.5 & 1171.7 & 86.6 & 0.0 & 150.9 \\
\hline Reduced & 93.1 & 301.5 & 1167.7 & 85.7 & 0.0 & 152.9 \\
\hline Difference & -2.3 & -2.0 & 4.0 & 0.9 & 0.0 & -2.0 \\
\hline
\end{tabular}

Contingency of Line 6

\begin{tabular}{|l|r|r|r|r|r|r|}
\hline Full & 53.2 & 271.3 & 1116.5 & 129.5 & 164.9 & 0.0 \\
\hline Reduced & 54.3 & 272.6 & 1121.2 & 130.2 & 164.3 & 0.0 \\
\hline Difference & -1.1 & -1.3 & -4.7 & -0.7 & 0.6 & 0.0 \\
\hline
\end{tabular}


The fifth variable compared was the reactive power output of the six lines under study for the same contingencies. The results of the comparison are given in Table 19 . The largest error observed was 1.3 MVAr on the Manitoba Hydro $500 \mathrm{kV}$ tie line for the loss of the tie line between North Western Ontario and USA (Line 6). 
Table 19: Line Reactive Power Comparison (MVAr)

\begin{tabular}{|c|c|c|c|c|c|c|}
\hline & Line 1 & Line 2 & Line 3 & Line 4 & Line 5 & Line 6 \\
\hline \multicolumn{7}{|c|}{ Pre contingency } \\
\hline Full & -11.800 & -29.200 & -56.700 & 2.100 & -3.700 & -16.700 \\
\hline Reduced & -12.000 & -29.200 & -56.600 & 2.300 & -3.900 & -16.700 \\
\hline Difference & 0.2 & 0 & -0.1 & -0.2 & 0.2 & 0 \\
\hline
\end{tabular}

Contingency of Line 1

\begin{tabular}{|l|r|r|r|r|r|r|}
\hline Full & 0.000 & -28.700 & -63.700 & 1.800 & -4.100 & -16.900 \\
\hline Reduced & 0.000 & -28.600 & -64.000 & 2.000 & -4.300 & -16.800 \\
\hline Difference & 0 & -0.1 & 0.3 & -0.2 & 0.2 & -0.1 \\
\hline
\end{tabular}

Contingency of Line 2

\begin{tabular}{|l|r|r|r|r|r|r|}
\hline Full & -10.200 & 0.000 & -65.700 & 2.200 & -4.000 & -16.600 \\
\hline Reduced & -10.400 & 0.000 & -66.500 & 2.400 & -4.400 & -16.700 \\
\hline Difference & 0.2 & 0 & 0.8 & -0.2 & 0.4 & 0.1 \\
\hline
\end{tabular}

Contingency of Line 3

\begin{tabular}{|l|r|r|r|r|r|r|}
\hline Full & -11.100 & -27.900 & 0.000 & 5.700 & -3.400 & -14.200 \\
\hline Reduced & -11.200 & -27.900 & 0.000 & 5.800 & -3.700 & -14.300 \\
\hline Difference & 0.1 & 0 & 0 & -0.1 & 0.3 & 0.1 \\
\hline
\end{tabular}

Contingency of Line 4

\begin{tabular}{|l|r|r|r|r|r|r|}
\hline Full & -11.800 & -29.500 & -56.500 & 0.000 & -4.200 & -20.200 \\
\hline Reduced & -12.000 & -29.500 & -56.500 & 0.000 & -3.500 & -20.700 \\
\hline Difference & 0.2 & 0 & 0 & 0 & -0.7 & 0.5 \\
\hline
\end{tabular}

Contingency of Line 5

\begin{tabular}{|l|r|r|r|r|r|r|}
\hline Full & -18.200 & -26.800 & -37.100 & 2.600 & 0.000 & -16.100 \\
\hline Reduced & -18.400 & -26.500 & -38.300 & 2.900 & 0.000 & -16.100 \\
\hline Difference & 0.2 & -0.3 & 1.2 & -0.3 & 0 & 0 \\
\hline
\end{tabular}

Contingency of Line 6

\begin{tabular}{|l|r|r|r|r|r|r|}
\hline Full & -11.800 & -29.300 & -52.800 & -2.700 & -4.100 & 0.000 \\
\hline Reduced & -12.100 & -29.200 & -51.500 & -2.600 & -3.800 & 0.000 \\
\hline Difference & 0.3 & -0.1 & -1.3 & -0.1 & -0.3 & 0 \\
\hline
\end{tabular}


Manitoba Hydro and the surrounding power system have four phase shifters connected to the system. Two of them are located on the Manitoba - Ontario interface. One is located on the Saskatchewan - USA interface and the other one is located on the North Western Ontario- USA interface. The behaviour of these phase shifters is very important for accurate calculation of power across the interface. Therefore, the phase shift angle was compared for the same set of contingencies. The results of the analysis are provided in Table 20. It can be seen that all phase shifters performed in an acceptable manner with the equivalent circuit. 
Table 20: Phase Shifter Angle Comparison (Degree)

\begin{tabular}{|c|c|c|c|c|}
\hline & PS1 & PS2 & PS3 & PS4 \\
\hline \multicolumn{5}{|c|}{ Pre contingency } \\
\hline Full & 65.5 & 38.4 & 38.4 & -18.9 \\
\hline Reduced & 64.5 & 37.4 & 37.4 & -19.3 \\
\hline Difference & 1.0 & 1.0 & 1.0 & 0.4 \\
\hline
\end{tabular}

Contingency of Line 1

\begin{tabular}{|l|r|r|r|r|}
\hline Full & 65.5 & 38.4 & 38.4 & -18.9 \\
\hline Reduced & 64.5 & 37.4 & 37.4 & -19.3 \\
\hline Difference & 1.0 & 1.0 & 1.0 & 0.4 \\
\hline
\end{tabular}

Contingency of Line 2

\begin{tabular}{|l|r|r|r|r|}
\hline Full & 65.5 & 38.4 & 38.4 & -20.3 \\
\hline Reduced & 64.5 & 37.4 & 37.4 & -20.9 \\
\hline Difference & 1.0 & 1.0 & 1.0 & 0.6 \\
\hline
\end{tabular}

Contingency of Line 3

\begin{tabular}{|l|r|r|r|r|}
\hline Full & 64.4 & 38.4 & 38.4 & -18.9 \\
\hline Reduced & 63.1 & 37.4 & 37.4 & -19.3 \\
\hline Difference & 1.3 & 1.0 & 1.0 & 0.4 \\
\hline
\end{tabular}

Contingency of Line 4

\begin{tabular}{|l|r|r|r|r|}
\hline Full & 63.3 & 40.5 & 40.5 & -18.9 \\
\hline Reduced & 60.8 & 38.7 & 38.7 & -19.3 \\
\hline Difference & 2.5 & 1.8 & 1.8 & 0.4 \\
\hline
\end{tabular}

Contingency of Line 5

\begin{tabular}{|l|r|r|r|r|}
\hline Full & 65.5 & 39.8 & 39.8 & -18.9 \\
\hline Reduced & 64.5 & 38.3 & 38.3 & -19.3 \\
\hline Difference & 1.0 & 1.5 & 1.5 & 0.4 \\
\hline
\end{tabular}

Contingency of Line 6

\begin{tabular}{|l|r|r|r|r|}
\hline Full & 65.5 & 20.4 & 20.4 & -18.9 \\
\hline Reduced & 64.5 & 19.4 & 19.4 & -19.3 \\
\hline Difference & 1.0 & 1.0 & 1.0 & 0.4 \\
\hline
\end{tabular}




\subsubsection{Use of Equivalent Circuit in Transfer Calculation}

The transfer level calculation is necessary to establish the system operating levels for various internal and external interfaces. Manitoba Hydro carries out off-line studies using the MRO model to establish transfer levels for all its internal and external interfaces including the Manitoba-Ontario, Manitoba-Saskatchewan and Manitoba-USA interfaces. The transfer level calculation usually starts with the MRO model. Once the MRO model is issued, Manitoba Hydro with the consultation of regional utilities carries out some changes to the original MRO model before starting transfer analysis. These adjustments may include adjusting transfer levels of various neighbouring interfaces to certain levels, adjusting load, adding any new system improvements which are not modelled, etc. The modified power flow, which is generally called the "base case", is then used in the transfer calculation.

The transfer level calculations usually involve increasing the transfer level in steps by adjusting generation and loads in the base case, and running a set of contingencies at each transfer level until any thermal or voltage limits are reached. This process is illustrated in Figure 41. 


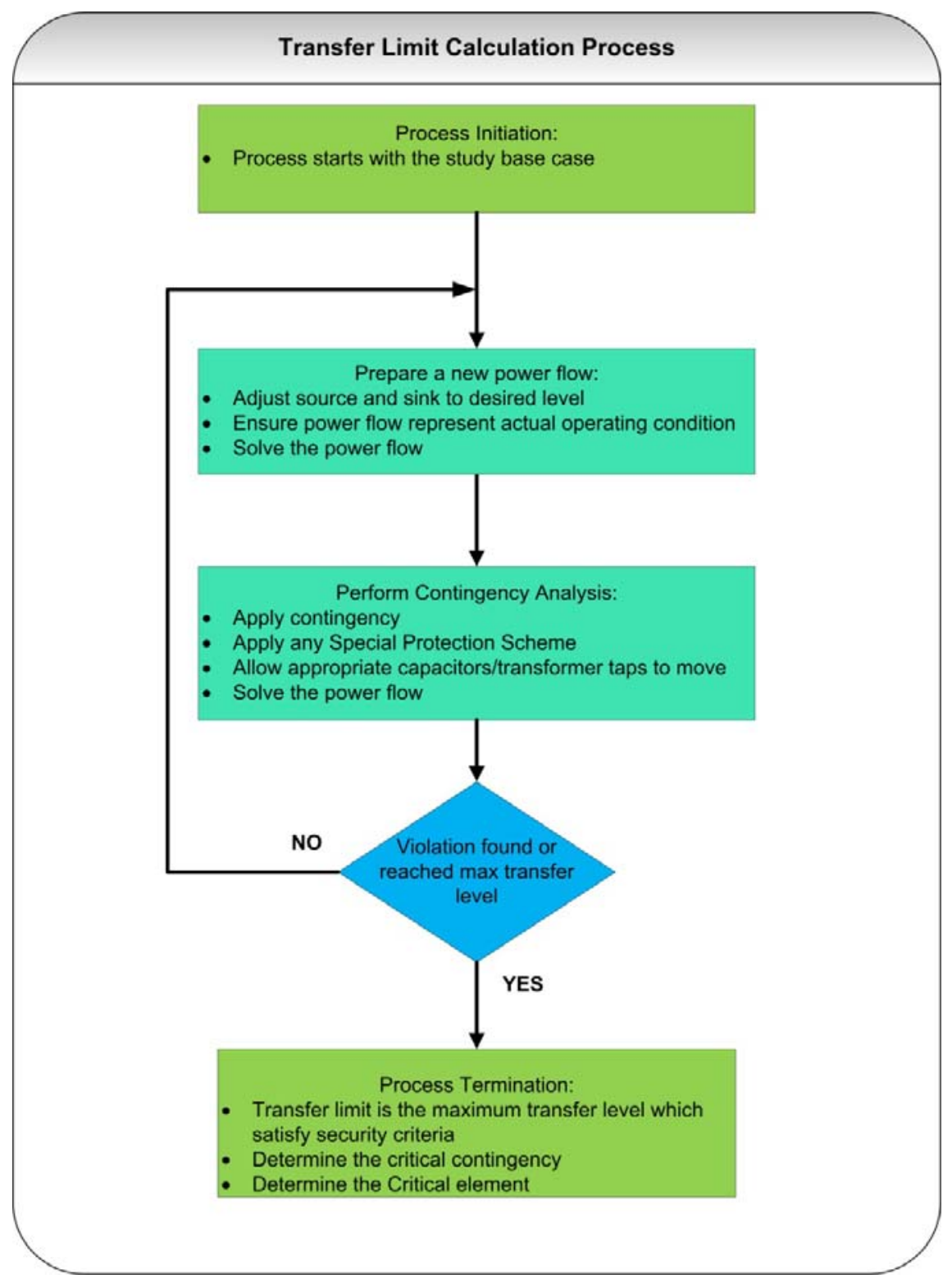

Figure 41: Transfer Limit Calculation Process

Manitoba Hydro is working towards implementing real-time transfer level calculations in Manitoba Hydro's control centre. As explained before, real-time transfer level calculations start from the power flow model derived from the EMS. Since Manitoba Hydro's EMS system does not represent the link between Manitoba and Ontario appropri- 
ately, it is necessary to use the equivalent circuit. Manitoba Hydro's base case used for the transfer calculation was cut off along the EMS boundary. The equivalent circuit developed using the proposed method was attached to the reduced model to prepare the reduced power flow model. The boundary matching procedure described in Chapter 3 was then used to map the internal line flow of the reduced model to be the same as the full model.

From the three external interfaces, the reduced EMS model greatly affected the Manitoba - USA interface. Therefore, transfer analysis was simulated for the Manitoba USA interface using the reduced power flow model and compared with the results of the transfer calculation for the full model.

Transfer level calculations were performed using the Manitoba Hydro - USA study package used to calculate Manitoba -USA transfer levels in the off-line studies. This package has a set of contingencies used in the transfer calculation, accurate modelling of all the components in the power system including phase shifter controls, SPS's, capacitors, and reactors behaviour.

The value of the transfer level for the Manitoba -USA interface was 2422 MW when the full off-line model was used to calculate the transfer level and was $2412 \mathrm{MW}$ when the reduced power flow model was used in the transfer level calculation. 
The transfer level of a particular interface is a function of the network configuration. When the network elements go out of service due to planned or forced outages, transfer levels of the interface can also change. This is also true for the Manitoba-USA interface. For example, if one tie line between Manitoba and USA is lost while Manitoba Hydro is exporting electricity to the USA, Manitoba Hydro cannot keep the same transfer level. Manitoba Hydro has to reduce the transfer level so that next element loss in the system does not jeopardise the power system. Due to that reason Manitoba Hydro calculates transfer capability for its interfaces for a number of prior outage conditions as well as for system intact. Transfer level calculations for prior outages involve additional steps to create a new base case considering the outages. Transfer level changes and the contingency analysis are then carried out on this new base case to determine the transfer level for the prior outage condition. The transfer level calculation process for prior outage conditions using the equivalent circuit is illustrated in Figure 42. 


\section{Transfer Limit Calculation Process for Prior Outage Conditions}

Process Initiation:

- Process starts with the study base case

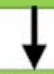

Create reduced power flow:

- Remove the boundary lines

- Add equivalent circuit, Perform the boundary matching

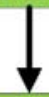

Create Prior Outage base case:

- Remove the line and solve the power flow

- Solve the power flow to generate new base case

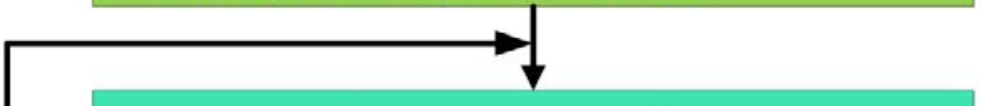

Prepare a new power flow:

- Adjust source and sink to desired level

- Ensure power flow represent actual operating condition

- Solve the power flow

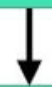

Perform Contingency Analysis:

- Apply contingency

- Apply any Special Protection Scheme

- Allow appropriate capacitors/transformer taps to move

- Solve the power flow

NO

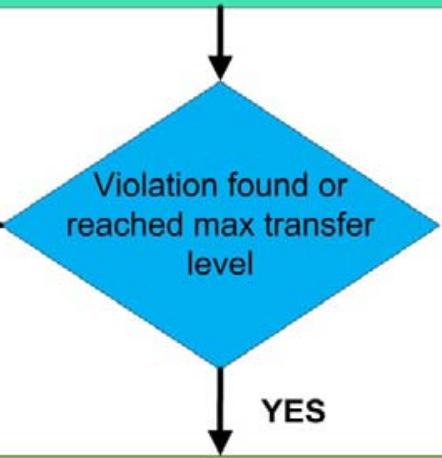

Process Termination:

- Transfer limit is the maximum transfer level which satisfy security criteria

- Determine the critical contingency

- Determine the Critical element

Figure 42: Transfer level Calculation Process for Prior Outage Condition 
Manitoba -USA transfer level was calculated for following six prior outages conditions:

- Prior outage of each of the tie lines between Manitoba and the USA (Line 1 to Line 4)

- Prior outage of the tie line between Saskatchewan and the USA (Line 5)

- Prior outage of the line between North western Ontario and the USA (Line 6)

A comparison of the transfer level calculation between the two models is shown in Table 21. It can be seen that the transfer level calculated using the two methods are very close except in two prior outage conditions. Those two are the prior outage of line 2 and line 3. Line 3 is the $500 \mathrm{kV}$ line between the Manitoba and the USA. These two scenarios were further analysed to find the reasons for the differences.

Table 21: Transfer Level Comparison between Two Models ((MW)

\begin{tabular}{|l|l|l|}
\hline Outage Condition & Using Full Model & Using Reduced Model \\
\hline System Intact & 2422 & 2412 \\
\hline Line 1 & 2180 & 2180 \\
\hline Line 2 & 1915 & 1942 \\
\hline Line 3 & 620 & 650 \\
\hline Line 4 & 2298 & 2300 \\
\hline Line 5 & 2403 & 2396 \\
\hline Line 6 & 2250 & 2235 \\
\hline
\end{tabular}


As mentioned above, Manitoba Hydro's study package used in the off-line studies was used to calculate transfer capability. Manitoba Hydro off-line transfer analysis starts with the base case which has a Manitoba-USA transfer level of $2500 \mathrm{MW}$. The Manitoba - USA study package has a program to reduce the transfer between the Manitoba - USA interface. The normal practice is reducing Manitoba Hydro northern island generation and DC while increasing generation in the USA. When creating the reduced model from the full model, some of those generators were cut off from the model. Other than creating an equivalent circuit between Manitoba and Ontario, no additional compensation was provided for the reduced generation/load in the USA area in the reduced model. This resulted in an increasing swing bus located in the USA with the reduction of the Manitoba - USA transfer level. This was the reason for the difference between the two models under those two prior outage conditions. When the swing bus power was distributed among the boundary the transfer level calculated using the equivalent model was much closer to the full model.

This exercise demonstrates that the reduced model can be use in transfer level calculation under system intact and a number of prior outage conditions. However, some prior outage conditions may require appropriate compensation for the generation and load lost due to the reduced model size. This is especially important if the transfer calculation requires large generation and or load manipulation from the current condition.

As described before, transfer calculation requires adjusting load and generation in different areas. Transfer adjustments should be carried out in the most realistic manner in 
order to get best results. Before the era of the electricity market, when the balancing area was small, most utilities knew the behavior of the generation dispatch pattern in the area. However, with the introduction of the electricity market most of the areas merged into one big balancing area. With this it is very difficult to determine the correct sink and source for transfer level calculations. To make the matter even worse, power systems today have lots of variable resources such as wind power generation. The outputs of these generators are highly unpredictable. Therefore, selecting correct source/sink pairs for transfer adjustment is not straightforward. Power system utilities should take time to analyze this problem before determining source/sink pairs in transfer calculations. Utilities should also continue to benchmark their models with real-time systems to improve their models.

\subsubsection{PV Analysis}

Accuracy of the equivalent circuit was also analyzed by carrying out PV analysis. PV analysis was started with the same set of base cases developed for the transfer level calculation which had a Manitoba-USA transfer level of 2500 MW. Manitoba-USA transfer levels were then reduced in steps of $100 \mathrm{MW}$ using the Manitoba - USA off-line transfer adjustment methods while monitoring the voltage of key Manitoba Hydro stations. The results of the analysis are shown in Figure 43. Voltage variation with the transfer level in the full model is shown in blue and voltage variation for the reduced model is shown in red. It can be seen that voltage profile between the two models is very close. The maximum voltage difference observed was only 0.0074 per unit. More importantly, this occurs 
in the first two transfer adjustments. Voltages between two models were very close in the last four transfer adjustments.
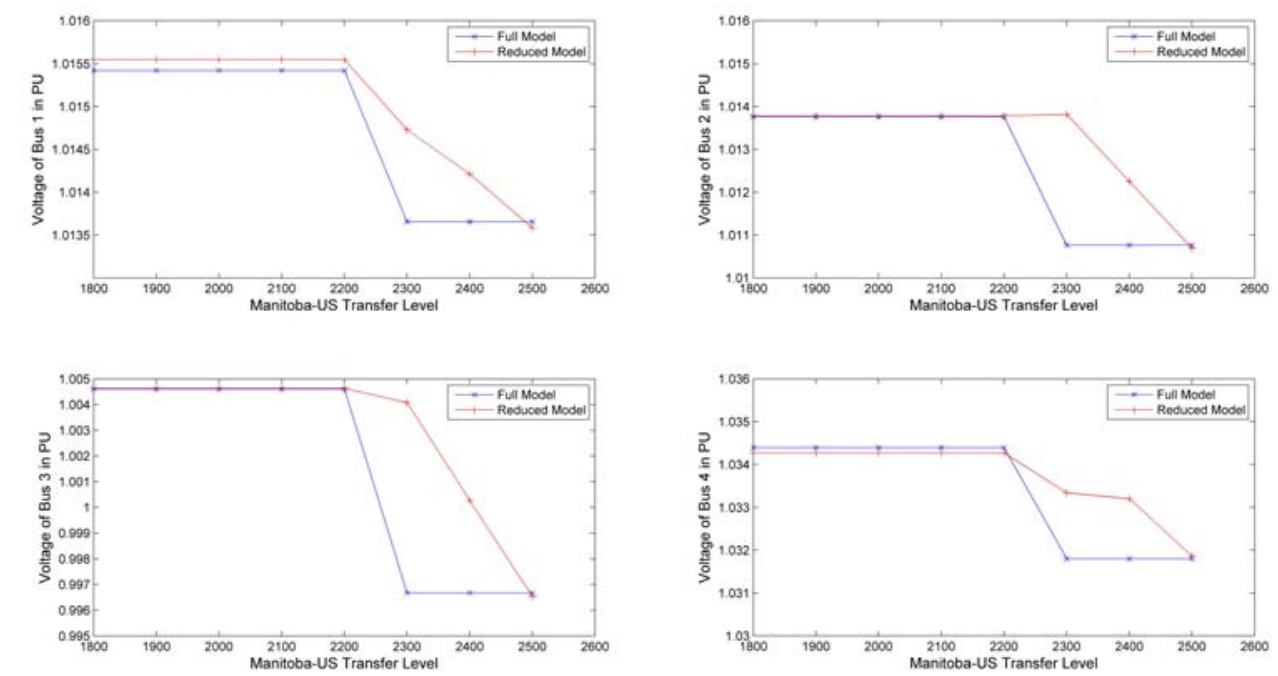

Figure 43: PV Analysis for Major Manitoba Stations. Variation of bus voltage for Station 1 to 4.

The same six contingencies selected for the contingency analysis were selected for the PV curves as well. The results of the analysis are shown from Figure 44 to Figure 49. Voltage variation with the transfer level in the full model is shown in blue and voltage variation for the reduced model is shown in red. It can be seen that all the PV curves provide comparable results. In some graphs, when the transfer level is increased, voltage suddenly increased. This is due to switching of some reactive control devices in the network. In Figure 46 and Figure 47 it appears that voltages profile of bus 3 is behaving differently in the full model and the reduced model. However, this is only highlighted due to 
the scale selected in the graphs. The voltages between two models are quite close to each other.
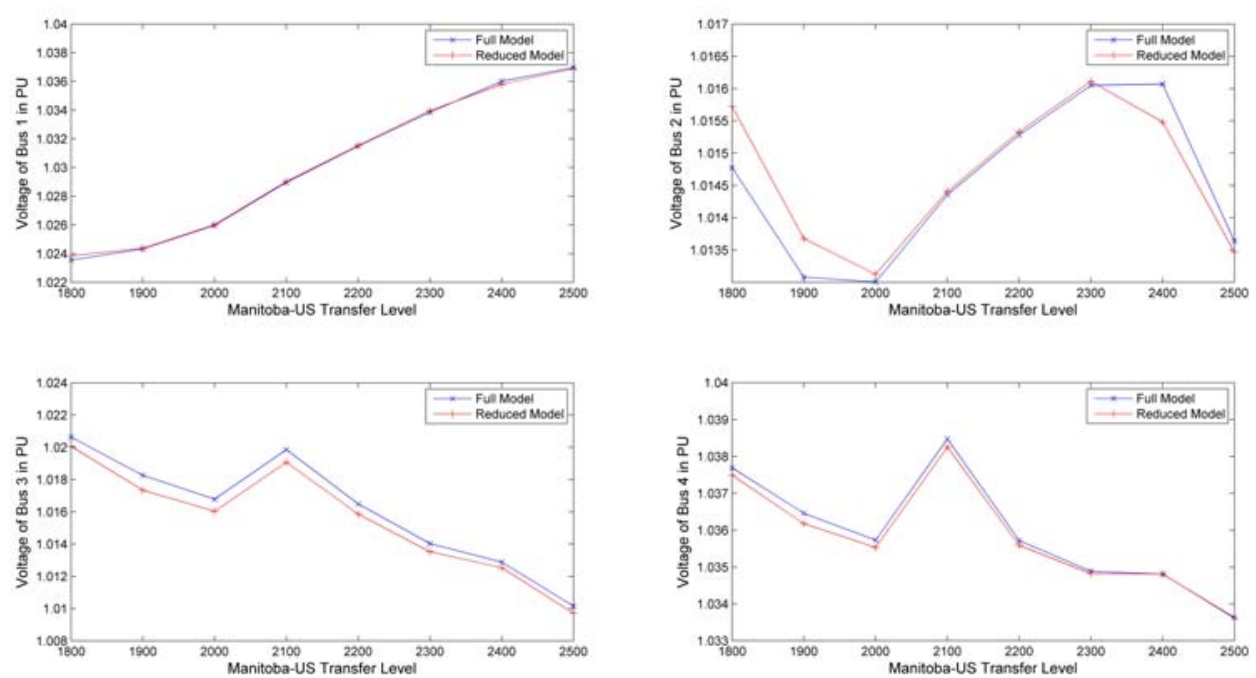

Figure 44: PV Analysis for Major Manitoba Stations. Variation of bus voltage for Station 1 to 4 for loss of Line 1 

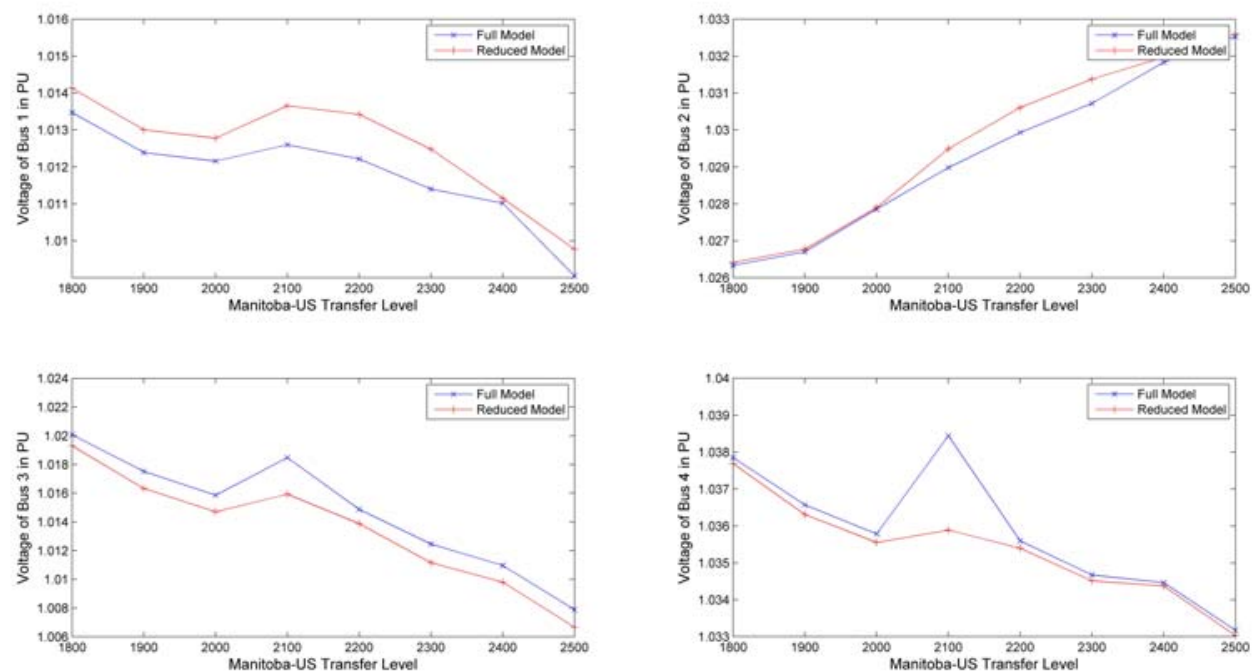

Figure 45: PV Analysis for Major Manitoba Stations. Variation of bus voltage for Station 1 to 4 for loss of Line 2
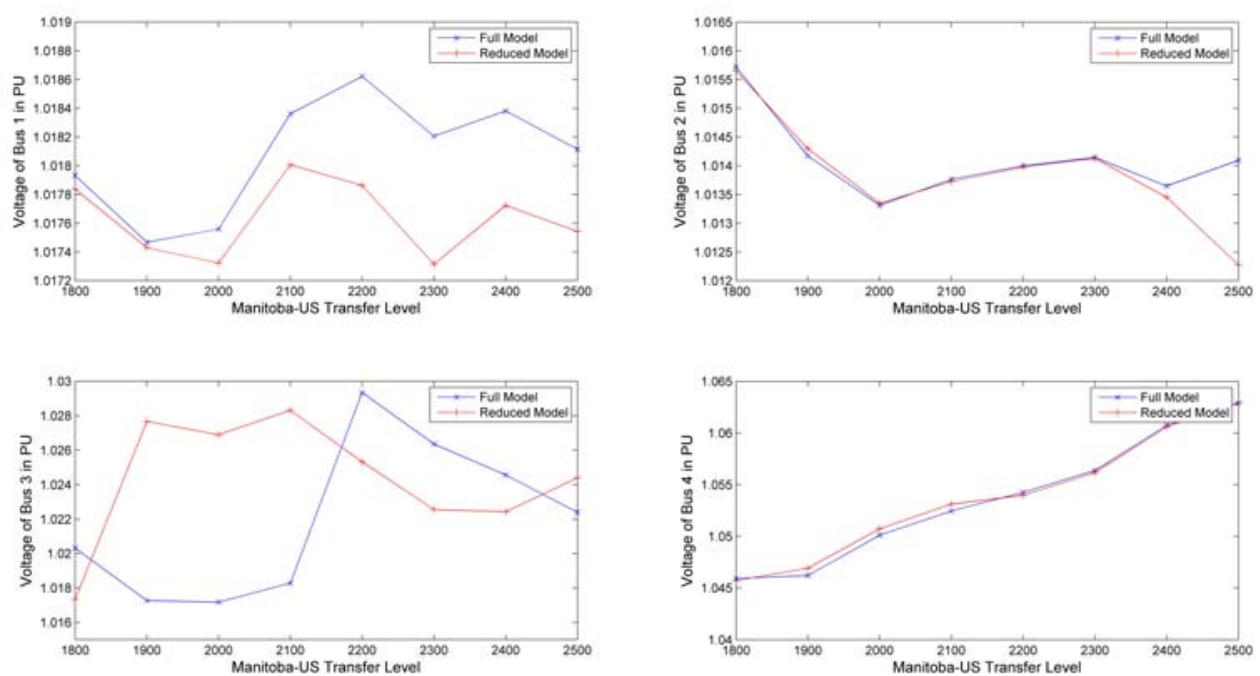

Figure 46: PV Analysis for Major Manitoba Stations. Variation of bus voltage for Station 1 to 4 for loss of Line 3 

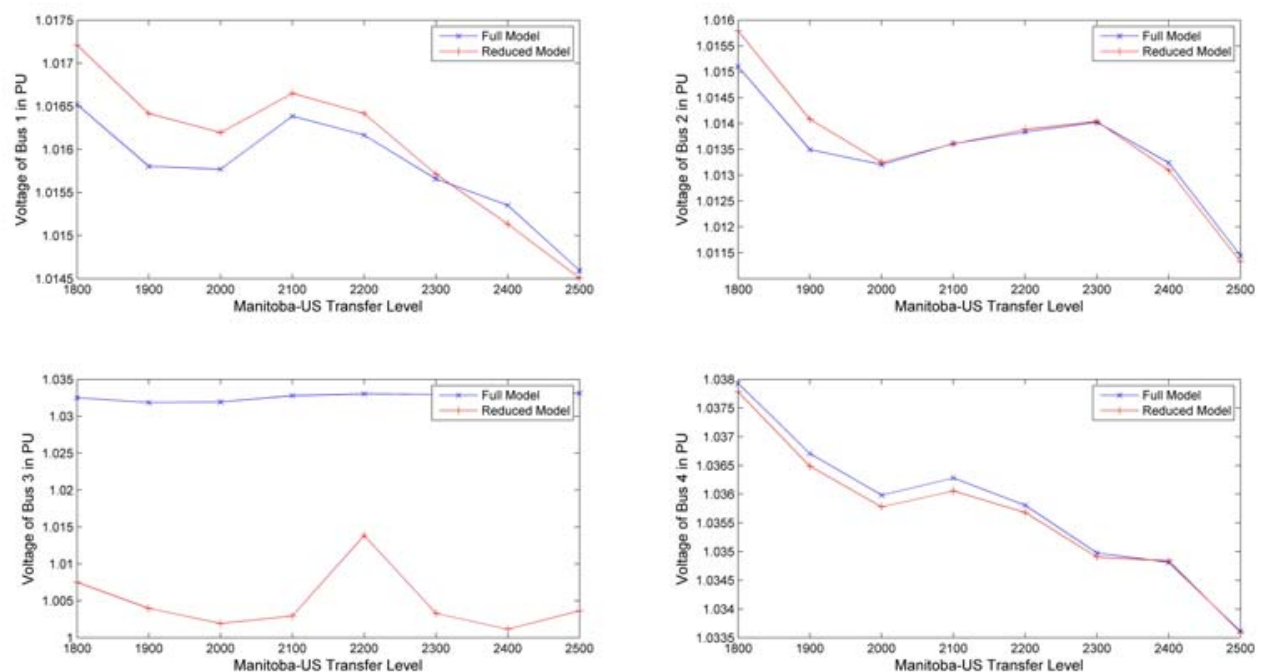

Figure 47: PV Analysis for Major Manitoba Stations. Variation of bus voltage for Station 1 to 4 for loss of Line 4
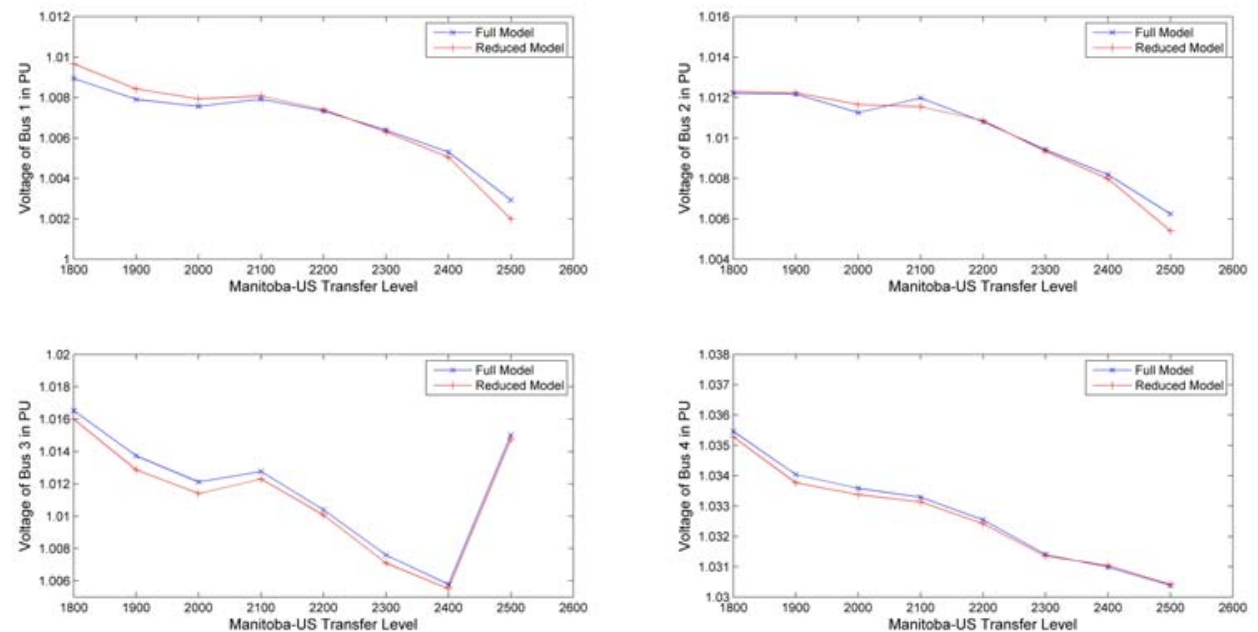

Figure 48: PV Analysis for Major Manitoba Stations. Variation of bus voltage for Station 1 to 4 for loss of Line 5 

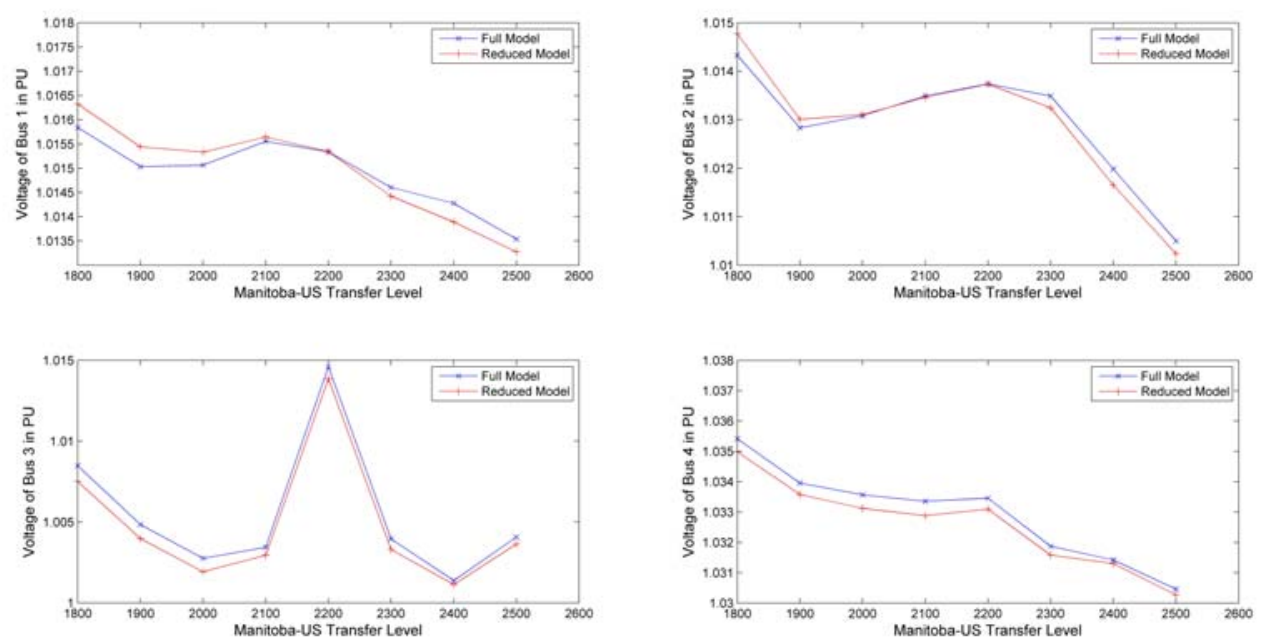

Figure 49: PV Analysis for Major Manitoba Stations. Variation of bus voltage for Station 1 to 4 for loss of Line 6

\subsection{Summary}

This chapter introduced Manitoba Hydro System and demonstrated the need to use the equivalent circuit in real-time security assessments. Proposed methodology was used to develop an equivalent circuit for the Manitoba Hydro system.

The equivalent circuit derived was benchmarked by performing contingency analysis, transfer analysis and PV analysis. Equivalent circuit demonstrates reasonable accuracy in all those scenarios. 


\section{Chapter 6}

\section{Proposed Improvements to Real-time}

\section{Limit Calculation Process}

Accurate modeling of power system response during a contingency is as important as developing a good power flow model. This chapter describes some of the possible improvements that can be carried out in the real-time limit calculation process.

\subsection{Contingency Analysis Associated with Load and Generation Loss}

One of the conditions must be satisfied in the security analysis of a power system is that, for any given island, the sum of power generated should be equal to the sum of the load connected to the system plus system losses. Off-line studies use the concept of a swing 
bus to balance the power system generation with load and losses. Typically one generator is selected as a swing machine for the island. This generator will adjust its output to balance the power in the island.

When the power system conditions change, the swing bus is used again to balance the power system. Traditionally, power systems engineers were only interested in simulating contingencies associated with transmission lines or transformers. Under these conditions, the difference between the pre-contingency power flow and post-contingency power flow is only the change of system losses due to a change of system configuration. Therefore, swing bus adjustments will be very small. Therefore, use of a swing bus under these circumstances does not introduce much error. However, recently more and more utilities are interested in simulating load losses and generation losses in their security assessments. Even under these circumstances, if the swing bus is located sufficiently far away from the study area this may not introduce much error.

Now let's look at the real-time security assessment. In real-time security assessments, the power system model comes from the utility's EMS model. Therefore, the area covered by the EMS power flow is much smaller than in off-line studies. This might prevent some utilities from moving the swing bus away from the study area. Under these circumstances the accuracy of using a swing bus during generation and load losses is questionable. 
If a generator or load is lost in the real-time system, initially all the generators connected to the island will respond to that disturbance based on their governor droop characteristics. Then the area responsible for the loss is responsible to provide adequate compensation based on the Area Control Error (ACE). For example, if a load is lost and that load cannot be brought back to the system immediately, generation in that area should be reduced accordingly to maintain the correct interchange flow with other areas. On the other hand, if a generator is lost and it cannot be brought on-line immediately, the nearby area is responsible in meeting its obligations by increasing generation or through activation of some reserve sharing agreement that may exist with other areas.

Most utilities have one or more generators designated as Automatic Generation Control (AGC) units. The function of these units is to maintain ACE at near zero with system changes.

Real-time studies engineers should take every effort to simulate the power system contingencies as close as possible to the system behavior. Under these circumstances, studies engineers should seriously reconsider the use of a swing bus during the contingencies associated with generation and load losses. Studies engineers should investigate the possibility of introducing governor and AGC behavior to power system simulations.

Some commercially available software has the capability to use governor or AGC action to balance the power flow instead of a swing bus. Unfortunately, it is not easy to select which method to use. This can be illustrated using few examples from Manitoba 
Hydro System. Figure 50 shows change of Manitoba Hydro total export during a loss one of its industrial customers. It can be seen that when Manitoba Hydro system lost the industrial customer, total Manitoba Hydro export increased by the amount equal to the lost customer load. However, within less than 2 min of the incident, Manitoba Hydro export came back to the original value. When Manitoba Hydro lost the industrial customer, Manitoba Hydro had extra generation. This extra generation initially flowed through the tie lines to neighbouring utilities. However, within less than 2 min, Manitoba Hydro AGC function managed to bring back the exports back to the original value. In order to simulate this incident accurately with the swing bus option, swing bus should be located inside the Manitoba Hydro area. On the other hand this event can be simulated accurately by modelling the AGC. 


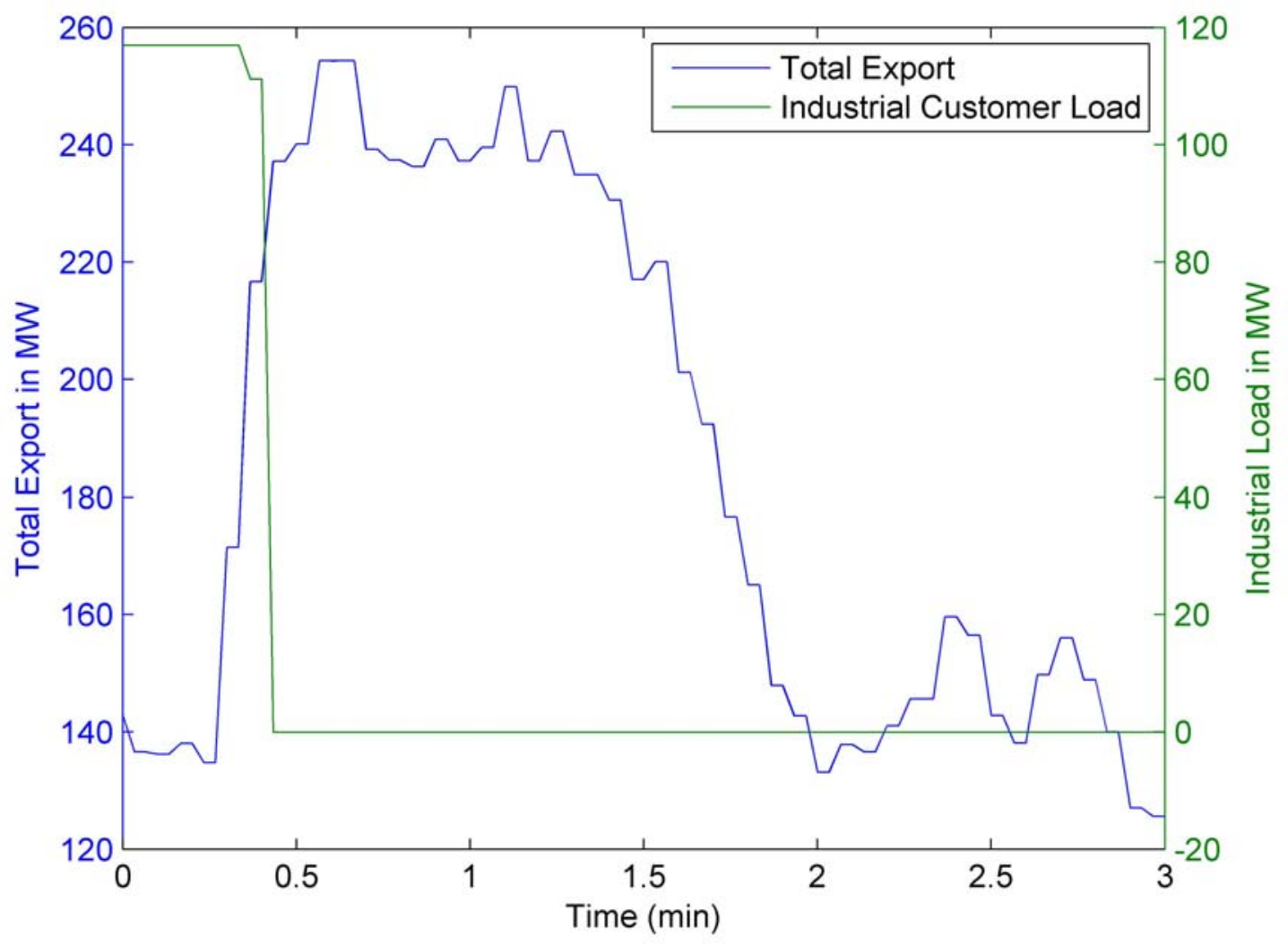

Figure 50: Change of Manitoba Hydro Total Export with a loss of industrial customer

Now consider another example, where Manitoba Hydro lost one of its tie lines with the United State of America. These tie lines have special protection schemes where when a tie line is lost, Manitoba Hydro dc is reduced by the amount of pre contingency flow on the tie line and AGC function is disabled. Manitoba Hydro system response at the time of loss of a tie line is shown in Figure 51. When Manitoba Hydro lost one of its tie lines, Manitoba Hydro total export was reduced by the pre contingency flow on the tie line. This event can be simulated accurately by located swing bus away from the Manitoba Hydro area or using governor option. 


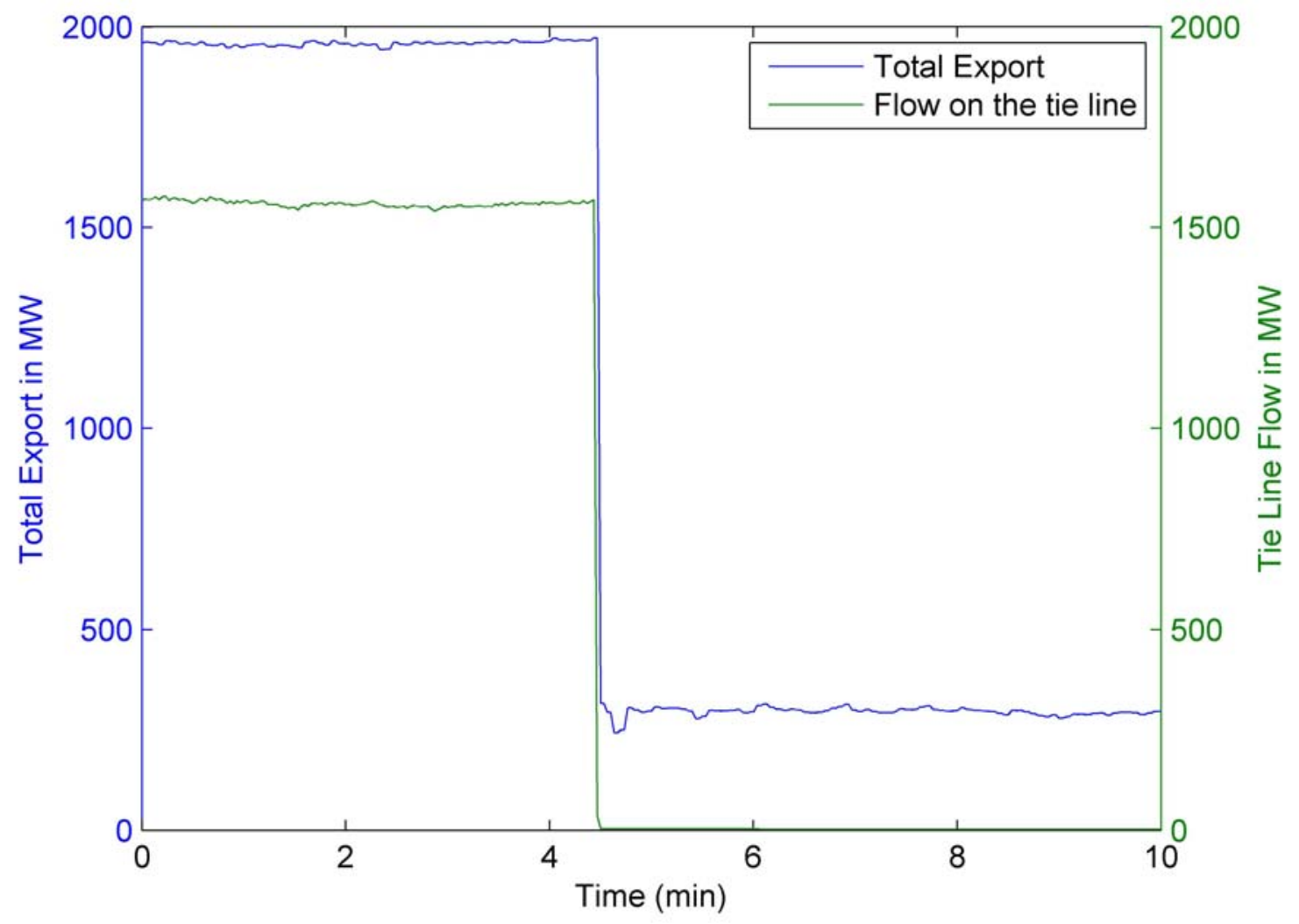

Figure 51: Change of Manitoba Hydro total export with loss of a tie line

Above two examples illustrated that using single swing bus, governor option or AGC option may not be able to provide accurate simulation response for all the contingencies. Therefore, provisions should be made to include both governor and AGC in the simulation similar to the actual system. Algorithm that can be used to simulate both governor and AGC response is shown in Figure 52.

The proposed algorithm involves solving post contingency power flow in several iterations to obtain final solution. In first iteration, power flow can be solved after applying contingency and any other Special Protection Schemes. If the contingency or SPS is 
associated with load or generation lost, the active power output of the swing bus will be changed. The change in the swing bus active power $\left(\mathrm{DP}_{\text {swing }}\right)$ then can be distributed among pre identified set of generators to simulate governor response. Power flow should then be solved to obtain governor response. At this stage it is necessary to evaluate any thermal or voltage violations. From Figure 50, it can be seen that immediately after the lost of industrial customer, Manitoba Hydro export was increased. The purpose of monitoring violation at governor time frame is to investigate the vulnerability of the system during this power surge. Relay loadability (Setting at which relay initiate trip function) or any other short term facility rating should be used to check the flow.

If the AGC function is enabled, this can be simulated by reversing the Governor dispatch and distributing swing bus active power difference among the AGC units to simulate the AGC response. This proposed approach will be able to provide an accurate response for different types of contingencies as mentioned above. 


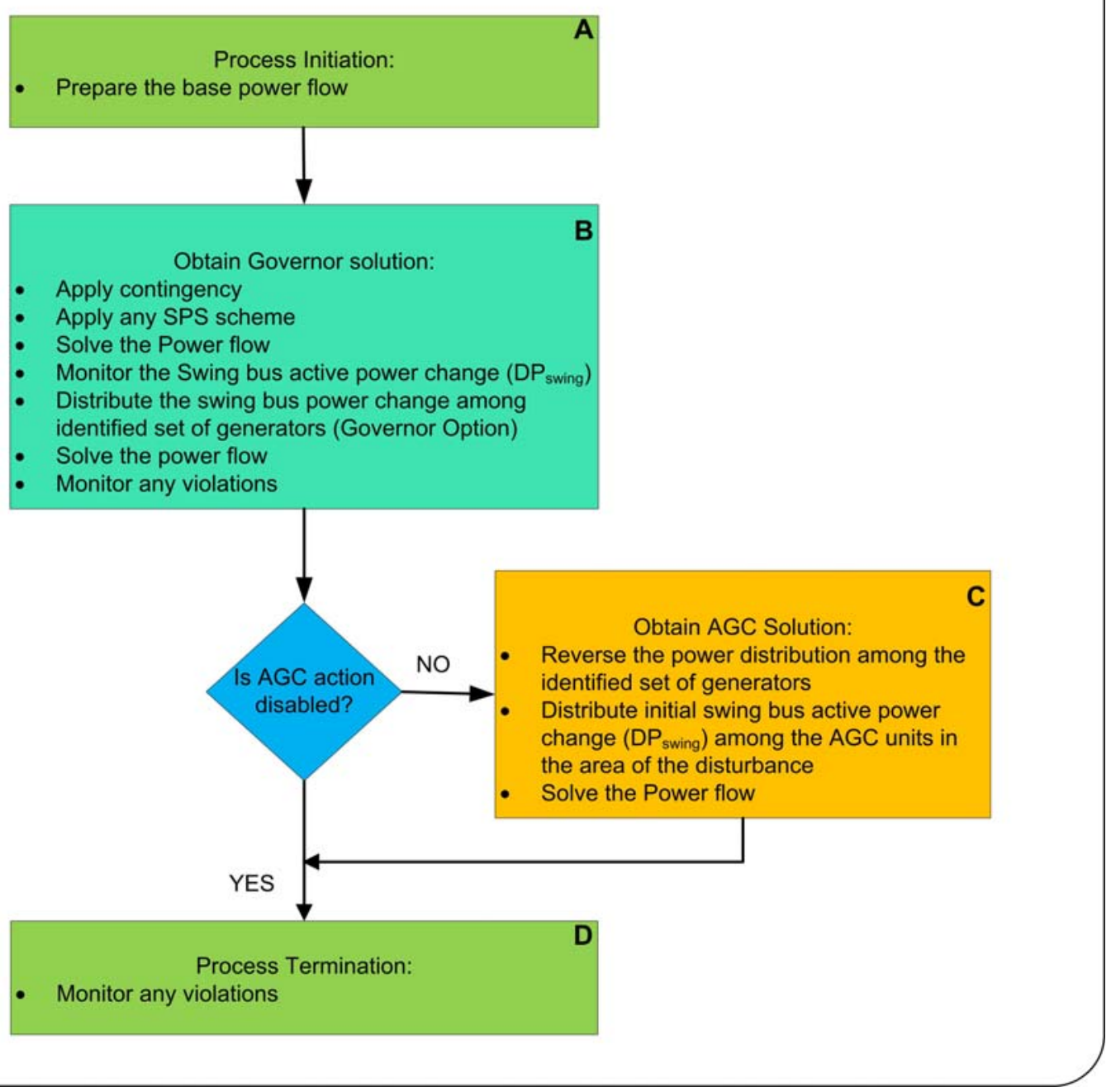

Figure 52: Algorithm to simulate both governor and AGC response

\subsection{System Response during Contingency Analy-}

\section{$\operatorname{sis}$}

Power system response to the disturbance is not limited to generators. Many other devices connected to the system can also respond to the disturbance. They include but are not limited to: switching reactive control devices, operation of various special protection 
systems, phase shifter adjustments, etc. These control actions need to be accurately modeled the real-time analysis. Under some circumstances, the order in which they operate is also important. In time domain simulation, modeling events in the correct sequence is straightforward. However, this is not trivial in non time domain simulations.

Some utilities including Manitoba Hydro solve the post-contingent power flow model in several steps to obtain the final-post contingent power flow. Each step is associated with control schemes such as activation of an SPS, phase shifter control, capacitors and reactor control, etc. However there is lots of customization and coordinating required to develop this kind of scheme. Therefore there is a need for a universally accepted approach where major power system software venders support this feature in their software.

Figure 53 shows a flow chart that can be used in real-time security assessments considering all the major components in the system, and governor and AGC response. The process starts with the real-time power flow received from the EMS. If necessary this power flow can be changed to reflect system conditions more accurately. Adding an equivalent circuit to represent an external area is one such example (Section A). 


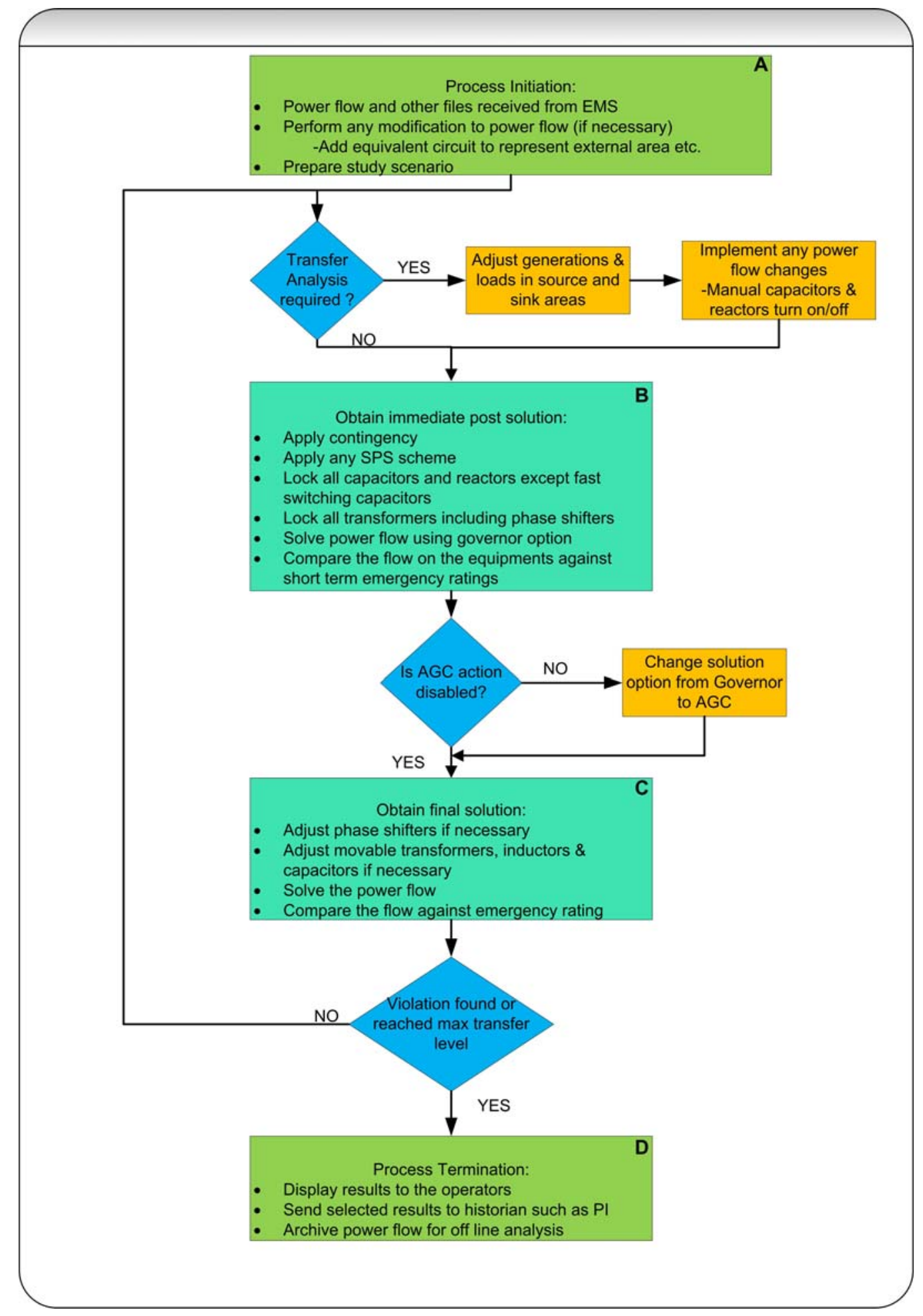

Figure 53: Proposed Real-time security assessment method 
If transfer analysis is required, then it is necessary to adjust power sources and sinks appropriately to develop a pre-contingency transfer scenario. Once this precontingency power flow model is prepared, it is very important to ensure that it represents the actual system behaviors. For example, a change in the transfer level may create certain bus voltages to be outside of the normal operating range. Yet, there may be capacitors or reactors close by which can control the voltage. However, if this capacitor or reactor is in manual mode, the power flow solution process may not switch that device. In real life, system operators always use capacitors and reactors to maintain system voltages at normal levels. Therefore, it is necessary to turn on any manual reactors or capacitors to bring the bus voltage to the normal range before starting contingency analysis.

The flowchart shows a two stage process for solving the post-contingency power flow model. Two stage solution processes are used in the some commercially available software. During this solution iteration, any contingencies are applied with any follow up SPS. All the capacitors, reactors and phase shifters are locked except any fast switching devices. The power flow is then solved using a governor solution. The resultant flow should then be compared against any short term emergency rating (section B). This process is lacking in the industry today. Some utilities including Manitoba Hydro check certain equipment in this solution step. However, not all equipment is checked. A recent NERC white paper on System Operating Limits (SOL) recommends the utilities to use SOL appropriately [155]. Most commercially available power flow simulation tools allow up to six ratings to be specified in the power flow. However, most software only uses two ratings in the security assessment. One rating is used to compare the steady state flow 
or the pre-contingency flow and other rating is use to compare the post-contingency flow. This should be changed to include at least three ratings to consider in the security assessment. In addition to the two ratings already used, the third rating should be used in the intermediate step of the post-contingency solution. If power system devices do not have short term ratings, relay settings can be used as the short term emergency rating.

The power flow solution then can be solved again using the governor solution and allowing all the other automatic devices in the system to move, including capacitors, reactors, phase shifters, etc (Section C). This process can be iterated until any violations are found.

Once the transfer calculation is performed, results should be displayed appropriately to the operators. Some of the selected results can be sent to a historian application such as PI. Power flow model and any of the other associated files can be stored for offline analysis (section D).

\subsection{Compensation for Reduced Generation and Loads in the Cut-off Area}

Section 5.3 demonstrates that when the equivalent circuit is used in transfer analysis where it is necessary to perform significant adjustment to generation and load, generation and load in the cut off area cannot be ignored. This section provides a small modification 
to the proposed equivalent circuit methodology that can be used under these circumstances.

Again consider the simple power system model used in chapter 2 . This power system is shown in Figure 54. The proposed method can be used to develop an equivalent circuit between the station $\mathrm{D} 3$ and $\mathrm{C} 1$ as shown in Figure 55.

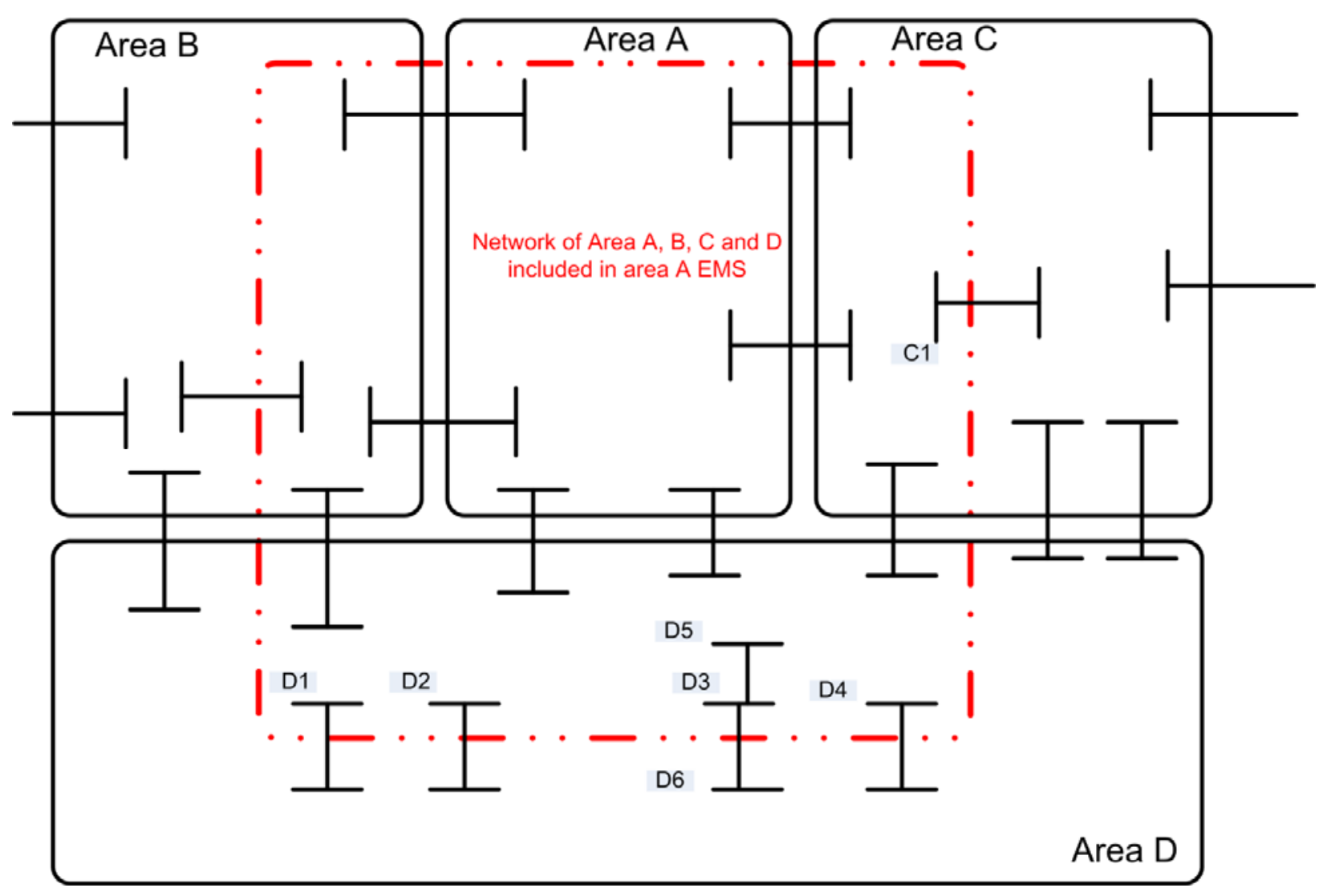

Figure 54: Part of the Interconnected System 


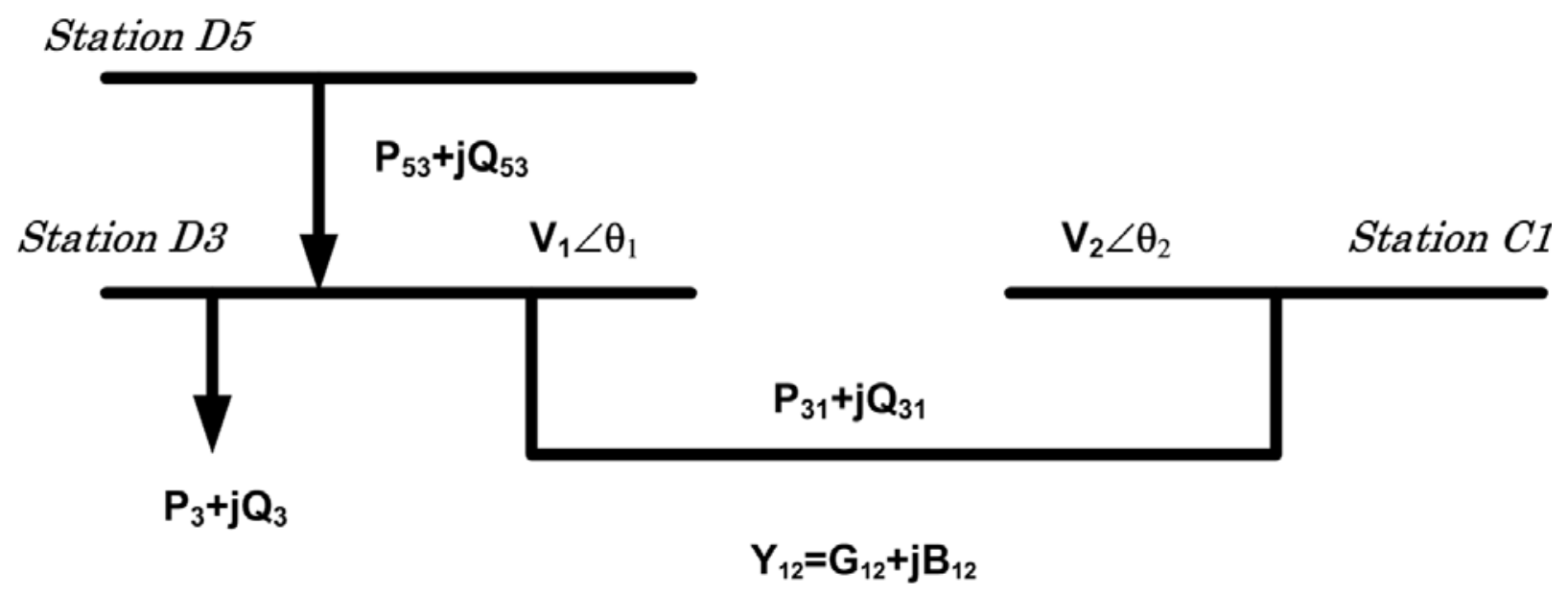

Figure 55: Proposed Equivalent system

In the equivalent circuit development, it was mentioned that the load connected to station D3 represents the actual load connected to station D3 as well as flow into the external area from the internal area. However, some contingencies may results in power coming from the external area into the internal area and this equivalent circuit cannot be used to represent this behavior. Therefore, it is proposed to add a generator at the selected boundary stations with the output of the generator set to zero. The active and reactive power limits of this generator should be carefully selected to represent the system behavior. When selecting generator capability and power coming from the external area, it is better to use past data gathered from actual system events rather than offline simulation results to obtain accurate system response.

Transfer analysis may sometime require significant adjustment to generation and load in the buffer zone. These changes sometime could change the behaviour of the buffer system. Due to this reason, some utilities would like to sink or source genera- 
tion/load at the boundary to facilitate the transfer. This can be achieved by adding load and generation at the boundary. If it is necessary to add load into boundary buses to facilitate transfer, power output of the generator connected to the boundary can set to the same as the boundary load value.

Most utilities carry out time domain dynamic simulations in addition to the static security assessments. For real-time dynamic simulation, it may be necessary to develop a dynamic equivalent circuit. One of the advantages of using a generator at the boundary stations is that, these generators can be used to represent the inertia of the external system.

\subsection{Summary}

This chapter proposed two enhancements that can improve the accuracy of the security assessment and one enhancement that can enhance the accuracy of transfer calculation, when it is necessary to adjust large amount of load or generation to calculate transfer level. 


\section{Chapter 7}

\section{Conclusions and Future Work}

This section presents the conclusion of the thesis and future works that can be carried out in this research area.

\subsection{Conclusions}

Today more and more utilities rely on real-time security assessments in power system operation. The accuracy of security assessments greatly depends on the data and the various assumptions and procedures used in the security assessment. The data used for the security assessment includes a power flow model derived based on real-time operating conditions. Utilities often use the power system network modelled in their EMS to prepare the real-time power flow model. This network usually covers the utility's own area, and some area immediately adjacent to its own area called the "Buffer Zone". The majority of the time, the area covered in this model is not sufficient to produce accurate secu- 
rity assessments. To fill this gap, most utilities use equivalent circuits created using offline studies to model the external area in their security assessments.

The subject of developing an equivalent circuit was studied by many researchers over the last 50 years. Some of the major previous research on this area were summarised in the Chapter 2. Almost all of the available methods require impedance information of the external area to develop an equivalent circuit. However, most utilities do not have access to this information in real-time. They use equivalent circuits created using off-line studies in their real-time model without proper validation. The accuracy of using the same equivalent circuit in all external operating conditions is highly questionable. Some utilities go one step further and produce multiple equivalent circuits based on the season, transfer levels, etc. This is better than using a single equivalent circuit. However, it may still not produce accurate results.

Chapter 3 of this thesis presented a simple yet effective method of deriving an equivalent circuit based on the boundary condition information. Unlike most of the existing methods like Ward and REI method, this method does not require impedance information of the external area to derive the equivalent circuit. This method only requires voltage magnitude and voltage angle of the boundary buses, and power going into the external area from the boundary buses. Utilities already have access to real-time boundary bus voltage and power information. Many utilities are now working towards adding PMU (Phaser Measurement Units) into their EMS's since in real-time operations it is impossible to use external network information to determine an equivalent circuit with changing network conditions. The proposed method provides a practical alternative to deriving an 
equivalent circuit using real-time boundary condition data from installed PMUs that can be adapted for varying system operating conditions.

Connection of an equivalent circuit to a power system model can sometimes change the active and reactive power flow values in the original system. Therefore it is necessary to carry out some form of boundary matching exercise so that boundary stations will provide same active and reactive power injection in the internal system. Section 3.2.1 proposed a simple boundary matching method which can be used to achieve this outcome.

Section 3.3 illustrated how the proposed methodology can be used to create an equivalent circuit for modified IEEE three area 300 bus system and how boundary matching can be performed after connecting equivalent circuit to avoid changes to the internal system states. The validity of the proposed methodology was tested by performing contingency analysis and comparing the results with the full model. The method was benchmarked against the impedance reduction method, which is one of the most common methods used in the industry today. Benchmarking results demonstrated that the proposed method could produce results very close to the impedance reduction method.

Even though the method suggested in Chapter 3 was published in a peer review journal [156], argument can be made this method is too specific and may not work in some power system networks. The proposed method cannot be also used to generate the equivalent circuit parameters between different stations in the same area. In order to ad- 
dress these issues a second method was proposed to calculate equivalent circuit parameters based on the boundary information. This method is presented in Chapter 4 and uses the least square method. The method was thoroughly benchmarked according to power system industry standards using contingency analysis, transfer analysis and PV analysis. All the analyses have shown that the developed equivalent circuit shows behaviour very similar to the full model and hence can be used in the security assessment.

In chapter 5, method proposed in Chapter 3 was used to create an equivalent circuit for the Manitoba Hydro system. The accuracy of the model was thoroughly investigated by performing contingency analysis including generation losses as high as 1500 MW, transfer analysis for system intact condition as well as number of prior outage conditions, and PV analyses.

The assumptions and methods used in a real-time assessment are as important as the power system model in producing accurate results. Power system studies traditionally used the concept of a swing bus to balance the power generated with the load and losses in a particular island. Traditional contingency analyses only evaluated the loss of transmission elements. They did not evaluate the impact of loss of load or generation. Under those circumstances, the use of a swing bus to balance the power flow does not produce much error. However, the use of a swing bus concept in real-time assessment where the swing bus may be located relatively close to the study area may not produce accurate results, particularly when considering contingencies associated with loss of load and generation. 
As an alternative, some commercially available power system simulation software use governor response and AGC response to balance the power flow. In the governor response, all the generators connected to the island participate in balancing generation, and in the AGC response, only the generators connected to the area responsible for the loss participate to balance the power. Even though this approach is better than the swing bus concept, sometimes it is not easy to choose between the governor and AGC options. This was illustrated in Chapter 6 using two system events that occured in the Manitoba Hydro system. Real-time systems experience both governor and AGC responses. Therefore, studies engineers should take every effort to model the correct system behaviour in simulations. Section 6.1 proposed a method where power flow solution can be used to obtain both governor and AGC solution. Unlike governor, AGC or swing bus option, this method can produce accurate simulation results for the different types of contingencies.

In addition to generators, other equipment and devices connected to the power system (such as special protection schemes, reactors, capacitors, transformers, and phase shifting transformers) also participate in determining the response of the power system after a disturbance. Different devices have different response times. Power system simulations will be more accurate if these devices can be modelled in the order in which they respond. This task is straightforward in time domain simulation. However, this is not trivial in static security assessment. Section, 6.2 of this thesis proposed a two-step solution process for solving post-contingency power flow model. An attempt was made to separate equipment connected to the power system into two steps. During the first step, it was 
proposed to solve the power flow using the governor response. In the second step, it was proposed to solve the power flow using the AGC option.

Utilities can use an equivalent circuit in real time transfer level calculation. Some of the transfer analysis may require significant adjustment of generation or load in the buffer zone to facilitate required transfer level. Since, these changes can impact the behaviour of the system; utilities prefer to adjust generation and loads in the boundary. This can be achieved by adding generators or loads at the boundary. However, care should be taken to set proper active and reactive power limits based on the actual system response.

In summary, this thesis presented a novel method that can be used to generate an equivalent circuit of a large interconnected power system for real-time security assessments. The method was tested for the IEEE 300 bus system as well as for Manitoba Hydro system. This work was published in IET Generation Transmission \& Distribution journal [156]. In contrast to other methods used by the industry, this method does not require impedance information of the external system to develop equivalent circuit and it only requires boundary bus information such as voltage magnitude, voltage angle and power coming in from external area. This boundary information is used to determine equivalent impedance between two areas. 


\subsection{Research Contributions}

Based on the concepts and results presented in this thesis, the main contributions of this work are as follows:

- Proposed a simple yet effective method to develop an equivalent circuit without using external system information. This method is based on the linear regression method and only uses boundary information. The method was validated with modified IEEE three area 300 bus system and Manitoba Hydro power system.

- Second method based on least square method was proposed to overcome some of the deficiencies associated with the first method. This method was thoroughly benchmarked according to power system industry standards by performing contingency analysis, transfer analysis and PV analysis.

- Boundary matching method which can be used to correct the active and reactive power injection from boundary stations in to internal system was proposed.

- The accuracy of using swing bus for the real time security assessment was questioned. It is also illustrated that the option to select Governor or AGC option will not work in some of the contingencies associated with generation and load losses. Therefore, it was proposed to include both governor and AGC option in the post disturbance analysis. This option was proposed to Powertceh and they will have this feature in the next VSAT version expected to release in April 2017. VSAT is one of the most popular real time security assessment tools used by the power industry around the world. 
- The importance of power system simulation reflecting actual system behaviour was highlighted. Attempt was made to group power system components into two groups based on their response time. This option was also presented to Powertech and they modified their VSAT V 16 to differentiate fast switching switch shunt devices (capacitors and reactors) for the rest of switch shunt devices.

These contributions led to the following publications in journals and conferences.

- Gayan Wijeweera, Udaya D. Annakkage, Wenjie Zhang, and Athula D. Rajapakse, "Derivation of an equivalent circuit for real-time security assessment." IET Generation, Transmission \& Distribution 10, no. 8 (2016): 1913-1920

- Gayan Wijeweera, Kevin J. Ostash, Wenjie Zhang, Allan D. Silk, "Implementation of real-time transfer limit calculation: Manitoba Hydro Experience", 2015 CIEGRE Canada conference.

- Gayan Wijeweera, Udaya D. Annakkage, Wenjie Zhang, and Athula D. Rajapakse, Michelle D. Rheault, "Development of an equivalent circuit of a large power system for real time security assessment". Submitted and presently addressing reviewer's comment for resubmission to IEEE Transactions on Power Systems.

\subsection{Future Work}

The method presented in this thesis only calculates the reactance of the equivalent circuit. The accuracy of the equivalent circuit may be increased by properly calculating the resistance of the equivalent circuit as well. 
Due to coordination required between various system operators to facilitate the sharing of real-time PMU data, it was not possible to create the equivalent circuit using real-time data. It is expected that system changes occurring due to changing system load and transfer levels can be used to generate the equivalent circuit in real-time. For example, Manitoba Hydro sells its excess energy to the USA market during peak hours of the day when the price is high and subsequently reduces the transfer during night when the price is low. The variation of voltage and voltage angle during power transfer scenarios needs to be analysed to produce the equivalent circuit in real-time.

This method assumes that all the boundary stations that need to be linked will have PMUs installed. However, in real systems it is possible that some of the boundary stations may not have PMU's installed. Therefore it is necessary to find a method to determine the voltage angle of those stations from the available PMU data.

In section 6.3, it was proposed to add generator at the boundary to account for the reduced generation in the cut-off area. However, this thesis does not describe a method to obtain various important parameters in those generators including maximum active and reactive power capability. It is proposed that real time system events should be used to determine these parameters instead of offline study results. However, further work is required in this area. 
This thesis only described steps involved in developing a static equivalent circuit. Many system operators carry out transient analysis as part of the transfer calculation as well. A dynamic equivalent circuit is required for these analyses. 


\section{Bibliography}

1. Wu, Felix F., Khosrow Moslehi, and Anjan Bose. "Power system control centers: Past, present, and future." Proceedings of the IEEE 93, no. 11 (2005): 1890-1908.

2. Savulescu, Savu Crivat. "Real-time stability assessment in modern power system control centers”. John Wiley \& Sons, 2009

3. Tomsovic, Kevin, David E. Bakken, Vaithianathan Venkatasubramanian, and Anjan Bose. "Designing the next generation of real-time control, communication, and computations for large power systems." Proceedings of the IEEE 93, no. 5 (2005): 965-979.

4. Dy-Liacco, Tomas E. "Control centers are here to stay." IEEE Computer Applications in Power 15, no. 4 (2002): 18-23.

5. Wang, L., P. P. Gelberger, and N. Ramani. "Reliability assessment of the operational functions of a power system control center." In Probabilistic Methods Applied to Electric Power Systems, 1991., Third International Conference on, pp. 229-234. IET, 1991. 
6. Bruce, A. G. "Reliability analysis of electric utility SCADA systems." In Power Industry Computer Applications., 1997. 20th International Conference on, pp. 200-205. IEEE, 1997.

7. Smith, Otto JM. "Power system state estimation." IEEE Transactions on Power Apparatus and Systems 3 (1970): 363-379.

8. Abur, Ali, and Antonio Gomez Exposito. Power system state estimation: theory and implementation. CRC press, 2004.

9. Handschin, Edmund, Fred C. Schweppe, Jurg Kohlas, and Armin Fiechter. "Bad data analysis for power system state estimation." IEEE Transactions on Power Apparatus and Systems 94, no. 2 (1975): 329-337.

10. Schweppe, Fred C., and Douglas B. Rom. "Power system static-state estimation, Part II: Approximate model." IEEE Transactions on Power Apparatus and Systems 1 (1970): 130-135.

11. Monticelli, A., 2000. Electric power system state estimation. Proceedings of the IEEE, 88(2), pp.262-282. .

12. Wu, F.F., 1990. Power system state estimation: a survey. International Journal of Electrical Power \& Energy Systems, 12(2), pp.80-87.

13. Mohagheghi, S., J. Stoupis, and Z. Wang. "Communication protocols and networks for power systems-current status and future trends." In Power Systems Conference and Exposition, 2009. PSCE'09. IEEE/PES, pp. 1-9. IEEE, 2009.

14. Robinson, J. T., T. Saxton, A. Vojdani, D. Ambrose, G. Schimmel, R. R. Blaesing, and R. Larson. "Development of the Intercontrol Center Communications Protocol (ICCP)[power system control]." In Power Industry Computer Ap- 
plication Conference, 1995. Conference Proceedings., 1995 IEEE, pp. 449-455. IEEE, 1995.

15. Ericsson, Goran. "Communication utilization in power system control. A state-ofthe-practice description." IEEE transactions on power delivery 13, no. 4 (1998): 984-989.

16. Soder, Lennart. "Reserve margin planning in a wind-hydro-thermal power system." IEEE Transactions on Power Systems 8, no. 2 (1993): 564-571.

17. Lee, Tsung-Ying. "Optimal spinning reserve for a wind-thermal power system using EIPSO." IEEE Transactions on Power Systems 22, no. 4 (2007): 1612-1621.

18. O'Sullivan, J. W., and M. J. O'Malley. "A new methodology for the provision of reserve in an isolated power system." IEEE transactions on power systems 14, no. 2 (1999): 519-524.

19. Ortega-Vazquez, Miguel A., and Daniel S. Kirschen. "Estimating the spinning reserve requirements in systems with significant wind power generation penetration." IEEE Transactions on Power Systems 24, no. 1 (2009): 114-124.

20. Ringlee, Robert J., and Allen J. Wood. "Frequency and duration methods for power system reliability calculations: II-Demand model and capacity reserve model." IEEE Transactions on Power Apparatus and Systems 4 (1969): 375-388.

21. Wang, Peng, and Roy Billinton. "Reliability assessment of a restructured power system considering the reserve agreements." IEEE Transactions on Power Systems 19, no. 2 (2004): 972-978.

22. Lopes, JA Peças, N. Hatziargyriou, J. Mutale, P. Djapic, and N. Jenkins. "Integrating distributed generation into electric power systems: A review of drivers, 
challenges and opportunities." Electric power systems research 77, no. 9 (2007): 1189-1203.

23. Gooi, H. B., D. P. Mendes, K. R. W. Bell, and D. S. Kirschen. "Optimal scheduling of spinning reserve." IEEE Transactions on Power Systems 14, no. 4 (1999): $1485-1492$.

24. Gaing, Zwe-Lee. "Particle swarm optimization to solving the economic dispatch considering the generator constraints." IEEE transactions on power systems 18 , no. 3 (2003): 1187-1195.

25. Lee, Fred N., and Arthur M. Breipohl. "Reserve constrained economic dispatch with prohibited operating zones." IEEE transactions on power systems 8 , no. 1 (1993): 246-254.

26. Su, Ching-Tzong, and Gwo-Jen Chiou. "A fast-computation Hopfield method to economic dispatch of power systems." IEEE Transactions on Power Systems 12, no. 4 (1997): 1759-1764.

27. Walsh, M. P., and M. J. O'malley. "Augmented Hopfield network for unit commitment and economic dispatch." IEEE Transactions on Power Systems 12, no. 4 (1997): 1765-1774.

28. Swarup, K. S., and S. Yamashiro. "Unit commitment solution methodology using genetic algorithm." IEEE Transactions on Power Systems 17, no. 1 (2002): 87-91.

29. Sheble, Gerald B., and George N. Fahd. "Unit commitment literature synopsis." IEEE Transactions on Power Systems 9, no. 1 (1994): 128-135.

30. Shoults, Raymond R., Show Kang Chang, Steve Helmick, and W. Mack Grady. "A practical approach to unit commitment, economic dispatch and savings alloca- 
tion for multiple-area pool operation with import/export constraints." IEEE Transactions on Power Apparatus and Systems 2 (1980): 625-635.

31. Sen, Subir, and D. P. Kothari. "Optimal thermal generating unit commitment: a review." International Journal of Electrical Power \& Energy Systems 20, no. 7 (1998): 443-451.

32. Ongsakul, Weerakorn, and Nit Petcharaks. "Unit commitment by enhanced adaptive Lagrangian relaxation." IEEE Transactions on Power Systems 19, no. 1 (2004): 620-628.

33. Snyder, Walter L., H. David Powell, and John C. Rayburn. "Dynamic programming approach to unit commitment." IEEE Transactions on Power Systems 2, no. 2 (1987): 339-348.

34. Jaleeli, Nasser, Louis S. VanSlyck, Donald N. Ewart, Lester H. Fink, and Arthur G. Hoffmann. "Understanding automatic generation control." IEEE transactions on power systems 7, no. 3 (1992): 1106-1122.

35. Kumar, Prabhat, and Dwarka P. Kothari. "Recent philosophies of automatic generation control strategies in power systems." IEEE transactions on power systems 20, no. 1 (2005): 346-357.

36. Christie, Richard D., and Anjan Bose. "Load frequency control issues in power system operations after deregulation." IEEE Transactions on Power Systems 11, no. 3 (1996): 1191-1200.

37. Nanda, J., and B. L. Kaul. "Automatic generation control of an interconnected power system." Electrical Engineers, Proceedings of the Institution of 125, no. 5 (1978): 385-390. 
38. Glavitsch, H., and J. Stoffel. "Automatic generation control." International Journal of Electrical Power \& Energy Systems 2, no. 1 (1980): 21-28.

39. Nanda, Janardan, Ashish Mangla, and Sanjay Suri. "Some new findings on automatic generation control of an interconnected hydrothermal system with conventional controllers." IEEE Transactions on energy conversion 21, no. 1 (2006): 187-194.

40. Bakken, Bjorn H., and Ove S. Grande. "Automatic generation control in a deregulated power system." IEEE Transactions on Power Systems 13, no. 4 (1998): 1401-1406.

41. Endsley, Mica R. "Toward a theory of situation awareness in dynamic systems." Human Factors: The Journal of the Human Factors and Ergonomics Society 37, no. 1 (1995): 32-64.

42. Wickens, Christopher D. "Situation awareness: Review of Mica Endsley's 1995 articles on situation awareness theory and measurement." Human Factors: The Journal of the Human Factors and Ergonomics Society 50, no. 3 (2008): 397-403.

43. Watts, Barry D. "9." Situation Awareness" in Air-to-Air Combat and Friction." McNair Papers 52-55 (1996): 91.

44. Endsley, Mica R. "Toward a theory of situation awareness in dynamic systems." Human Factors: The Journal of the Human Factors and Ergonomics Society 37, no. 1 (1995): 32-64.

45. Hogg, David N., K. N. U. T. FOLLES $\oslash$, Frode Strand-Volden, and Belén Torralba. "Development of a situation awareness measure to evaluate advanced alarm 
systems in nuclear power plant control rooms." Ergonomics 38, no. 11 (1995): 2394-2413.

46. Morison, Kip, Lei Wang, and Prabha Kundur. "Power system security assessment." IEEE Power and Energy Magazine 2, no. 5 (2004): 30-39.

47. Balu, Neal, Timothy Bertram, Anjan Bose, Vladimir Brandwajn, Gerry Cauley, David Curtice, Aziz Fouad et al. "On-line power system security analysis." Proceedings of the IEEE 80, no. 2 (1992): 262-282.

48. Savulescu, Savu Crivat. Real-time stability assessment in modern power system control centers. Vol. 42. John Wiley \& Sons, 2009.

49. Marceau, R. J., J. Endrenyi, R. Allan, F. L. Alvarado, G. A. Bloemhof, T. Carlsen, G. Couto et al. "Power system security assessment: a position paper." Electra 175 (1997): 49-77.

50. U.S. Federal Energy Commission, "Final report on 1965 blackout," July 19, 1967.

51. Garcia, Freddy, N. D. R. Sarma, Venkata Kanduri, Greeshma Nissankala, Karthik Gopinath, Jithender Polusani, Tim Mortensen, and Isabel Flores. "ERCOT control center experience in using real-time contingency analysis in the new nodal market." In 2012 IEEE Power and Energy Society General Meeting, pp. 1-8. IEEE, 2012.

52. Dondeti, J. R., C. Yang, K. Trotter, A. Witmeier, and K. Sherd. "Experiences with contingency analysis in reliability and market operations at MISO." In 2012 IEEE Power and Energy Society General Meeting, pp. 1-7. IEEE, 2012. 
53. Wangen, Brett, and Hongming Zhang. "Monitoring for post-contingency system operating limit exceedance in the western interconnection." In 2012 IEEE Power and Energy Society General Meeting, pp. 1-6. IEEE, 2012.

54. Thompson, C., K. McIntyre, Sarma Nuthalapati, A. Garcia, and Elias A. Villanueva. "Real-time contingency analysis methods to mitigate congestion in the ercot region." In 2009 IEEE Power \& Energy Society General Meeting, pp. 17. IEEE, 2009.

55. Tong, J. "Real time transfer limit calculations." In Power Engineering Society Summer Meeting, 2000. IEEE, vol. 2, pp. 1297-1302. IEEE, 2000.

56. Grainger, John J., and William D. Stevenson. Power system analysis. McGrawHill, 1994.

57. Saadat, Hadi. Power system analysis. WCB/McGraw-Hill, 1999.

58. Glover, J. Duncan. Power systems analysis and design. PWS Publishing Co., 1987.

59. Midwest reliability Organization Model Building Manual.

60. Geisler, Kenneth I., and Anjan Bose. "State estimation based external network solution for on-line security analysis." IEEE Transactions on Power Apparatus and Systems 8 (1983): 2447-2454.

61. Debs, A. S. "Estimation of steady-state power system model parameters." IEEE Transactions on Power Apparatus and Systems 5 (1974): 1260-1268.

62. Debs, A. S. "Estimation of external network equivalents from internal system data." IEEE Transactions on Power Apparatus and Systems 94, no. 2 (1975): 273279. 
63. Dopazo, J. F., M. H. Dwarakanath, J. J. Li, and A. M. Sasson. "An external system equivalent model using real-time measurements for system security evaluation." IEEE Transactions on Power Apparatus and Systems 96, no. 2 (1977): 431446.

64. Bose, Anjan. "Modeling of external networks for on-line security analysis." IEEE transactions on power apparatus and systems 8 (1984): 2117-2125.

65. Lu, C. N., K. C. Liu, and S. Vemuri. "An external network modeling approach for online security analysis." IEEE Transactions on Power Systems 5, no. 2 (1990): $565-573$.

66. Kim, Hongrae, and Ali Abur. "Enhancement of external system modeling for state estimation [power systems]." IEEE transactions on power systems 11, no. 3 (1996): 1380-1386.

67. Monticelli, A., and Felix F. Wu. "A method that combines internal state estimation and external network modeling." IEEE transactions on power apparatus and systems 1 (1985): 91-103.

68. Hazen, Harold Locke, O. R. Schurig, and Murray F. Gardner. "The mit network analyzer design and application to power system problems." Transactions of the American Institute of Electrical Engineers 49, no. 3 (1930): 1102-1113.

69. Morgan, W. A., F. S. Rothe, and J. J. Winsness. "An Improved AC Network Analyzer." Transactions of the American Institute of Electrical Engineers 68, no. 2 (1949): 891-897.

70. Bobo, P. O. "Handling power system problems on an ac network calculator." Electrical Engineering 69, no. 10 (1950): 867-869. 
71. C. Concordia and H. A. Peterson "ANALYZERS", General Electric Review (Schenectady, N. Y.), pp.29 -37 1945

72. https://en.wikipedia.org/wiki/Network_analyzer_(AC_power)

73. Ward, James B. "Equivalent circuits for power-flow studies." Electrical Engineering 68, no. 9 (1949): 794-794.

74. Brown, W. T., and W. J. Cloues. "Combination Load-Flow and Stability Equivalent for Power System Representation on AC Network Analyzers [includes discussion]." Transactions of the American Institute of Electrical Engineers. Part III: Power Apparatus and Systems 74, no. 3 (1955). 782-787.

75. Jennings, Philip D., and George E. Quinan. "The use of business machines in determining the distribution of load and reactive components in power line networks." Electrical Engineering 65, no. 12 (1946): 1045-1046.

76. Dunstan, Lyle A. "Machine computation of power network performance." Transactions of the American Institute of Electrical Engineers 66, no. 1 (1947): 610624.

77. Bennett, J. M., F. V. Dakin, and U. G. Knight. "Digital Computers and their Application to Some Electrical Engineering Problems'." CIGRE, Paris (1952). Paper No. 304.

78. Dunstan, Lyle A. "Digital Load Flow Studies [includes discussion]." Transactions of the American Institute of Electrical Engineers. Part III: Power Apparatus and Systems 73, no. 1 (1954). 
79. Henderson, J. M. "Automatic Digital Computer Solution of Load Flow Studies [includes discussion]." Transactions of the American Institute of Electrical Engineers. Part III: Power Apparatus and Systems 73, no. 2 (1954).

80. Bennett, J. M. "Digital computers and the load-flow problem." Proceedings of the IEE-Part B: Radio and Electronic Engineering 103, no. 1 (1956): 16-25.

81. Ward, J. B., and H. W. Hale. "Digital computer solution of power-flow problems [includes discussion]." Transactions of the American Institute of Electrical Engineers. Part III: Power Apparatus and Systems 75, no. 3 (1956).

82. Brown, Rodney J., and William F. Tinney. "Digital solutions for large power networks." Transactions of the American Institute of Electrical Engineers. Part III: Power Apparatus and Systems 76, no. 3 (1957): 347-351.

83. Glimn, A. F., and G. W. Stagg. "Automatic calculation of load flows." Transactions of the American Institute of Electrical Engineers. Part III: Power Apparatus and Systems 76, no. 3 (1957): 817-825.

84. Johnson, David L., and J. B. Ward. "The Solution of Power System Stability Problems by Means or Digital Computers [includes discussion]." Transactions of the American Institute of Electrical Engineers. Part III: Power Apparatus and Systems 75 , no. 3 (1956).

85. Van Ness, James E. "Iteration methods for digital load flow studies." Transactions of the American Institute of Electrical Engineers. Part III: Power Apparatus and Systems 78, no. 3 (1959): 583-586. 
86. Gupta, P. P., and MW Humphrey Davies. "Digital computers in power system analysis." Proceedings of the IEE-Part A: Power Engineering 108, no. 41 (1961): 383-398.

87. Laughton, M. A., and MW Humphrey Davies. "Numerical techniques in solution of power-system load-flow problems." In Proceedings of the institution of electrical engineers, vol. 111, no. 9, pp. 1575-1588. IET Digital Library, 1964.

88. Sasson, Albert M., and Fernando J. Jaimes. "Digital methods applied to power flow studies." IEEE Transactions on Power Apparatus and Systems 7 (1967): $860-867$.

89. Tinney, William F., and Clifford E. Hart. "Power flow solution by Newton's method." IEEE Transactions on Power Apparatus and systems 11 (1967): 14491460.

90. Freris, L. L., and A. M. Sasson. "Investigation of the load-flow problem." Electrical Engineers, Proceedings of the Institution of 115, no. 10 (1968): 1459-1470.

91. Stott, Brian. "Review of load-flow calculation methods." Proceedings of the IEEE 62, no. 7 (1974): 916-929.

92. Duran, Hernando, and N. V. Arvanitidis. "Simplifications for area security analysis: A new look at equivalence." IEEE Transactions on Power Apparatus and Systems 2 (1972): 670-679.

93. Stagg, Glenn, Jorge Dopazo, Oscar Klitin, and Louis Vanslyck. "Techniques for the real-time monitoring of power system operations." IEEE transactions on power Apparatus and Systems 4, no. PAS-89 (1970): 545-555.. 
94. Dopazo, J., O. Klitin, G. Stagg, and L. Van Slyck. "State calculation of power systems from line flow measurements." IEEE transactions on power Apparatus and Systems 7, no. PAS-89 (1970): 1698-1708.

95. Liacco, TE Dy. "Real-time computer control of power systems." Proceedings of the IEEE 62, no. 7 (1974): 884-891.

96. Deckmann, S., A. Pizzolante, A. Monticelli, B. Stott, and O. Alsac. "Studies on power system load flow equivalencing." IEEE Transactions on power Apparatus and Systems 6, no. PAS-99 (1980): 2301-2310.

97. Deckmann, Sua, A. Pizzolante, A. Monticelli, B. Stott, and O. Alsac. "Numerical testing of power system load flow equivalents." IEEE Transactions on Power Apparatus and Systems 6 (1980): 2292-2300.

98. Wu, Felix Fulih, and A. Monticelli. "Critical review of external network modelling for online security analysis." International Journal of Electrical Power \& Energy Systems 5, no. 4 (1983): 222-235.

99. Snyder Jr, W.L., Vemuri, S., Papalexopoulos, A.D., Oatts, M.L., Contaxis, G.C., Singh, J., Smith, R.A. and Savulescu, S.C., 1994. External network modeling-Recent practical experience. IEEE Transactions on Power Systems (Institute of Electrical and Electronics Engineers);(United States), 9(1).

100. Rahimi, A. F., K. Kato, S. H. Ansari, V. Brandwajn, G. Cauley, and D. J. Sobajic. "On external network model development." IEEE transactions on power systems 11, no. 2 (1996): 905-910. 
101. Monticelli, A., S. Deckmann, A. Garcia, and B. Stott. "Real-time external equivalents for static security analysis." IEEE Transactions on Power Apparatus and Systems 2 (1979): 498-508.

102. Lo, K. L., L. J. Peng, J. F. Macqueen, A. O. Ekwue, and N. H. Dandachi. "Extended Ward equivalent of external system for on-line security analysis." In Advances in Power System Control, Operation and Management, 1993. APSCOM93., 2nd International Conference on, pp. 54-59. IET, 1993.

103. Aschmoneit, F. C., and J. F. Verstege. "An external system equivalent for online steady-state generator outage simulation." IEEE Transactions on Power Apparatus and Systems 3 (1979): 770-779.

104. Van Amerongen, R. A. M., and H. P. Van Meeteren. "A generalised Ward equivalent for security analysis." IEEE Transactions on Power Apparatus and Systems 6 (1982): 1519-1526.

105. Machowski, J., A. Cichy, F. Gubina, and P. Omahen. "External subsystem equivalent model for steady-state and dynamic security assessment." IEEE Transactions on power systems 3, no. 4 (1988): 1456-1463.

106. Shoults, Raymond R., and William J. Bierck. "Buffer system selection of a steady-state external equivalent model for real-time power flow using an automated sensitivity analysis procedure." IEEE transactions on power systems 3 , no. 3 (1988): 1104-1111..

107. Snyder, W.L., 'Load flow equivalent circuits. An overview', IEEE PES Winter Meeting, New York, NY, 1972, pp. 436-444.

108. P. Dimo, Nodal Analysis of Power Systems, Abacus Press, England. 1975. 
109. Tinney, W. F., and W. L. Powell. "The REI approach to power network equivalents." In Proceedings of the PICA Conference, Toronto, pp. 314-320. 1977

110. Liacco, TE Dy, S. C. Savulescu, and K. A. Ramarao. "An on-line topological equivalent of a power system." IEEE Transactions on Power Apparatus and Systems 5, no. PAS-97 (1978): 1550-1563.

111. Irisarri, G., A. Sasson, and J. Dopazo. "Real-time external system equivalent for on-line contingency analysis." IEEE Transactions on Power Apparatus and Systems 6, no. PAS-98 (1979): 2153-2171.

112. Savulescu, Savu C. "Equivalents for security analysis of power systems." IEEE Transactions on Power Apparatus and Systems 5 (1981): 2672-2682.

113. Housos, Efthymios, and Guillermo Irisarri. "Real time results with on-line network equivalents for control center applications." IEEE Transactions on Power Apparatus and Systems 12 (1981): 4830-4837.

114. Housos, E., G. Irisarri, R. Porter, and A. Sasson. "Steady state network equivalents for power system planning applications." IEEE Transactions on Power Apparatus and Systems 6, no. PAS-99 (1980): 2113-2120.

115. Wu, F. F., and N. Narasimhamurthi. "Necessary conditions for REI reduction to be exact." In IEEE TRANSACTIONS ON POWER APPARATUS AND SYSTEMS, vol. 98, no. 4, pp. 1144-1144. 345 E 47TH ST, NEW YORK, NY 100172394: IEEE-INST ELECTRICAL ELECTRONICS ENGINEERS INC, 1979.

116. Tinney, William F., and Joseph M. Bright. "Adaptive reductions for power flow equivalents." IEEE transactions on power systems 2, no. 2 (1987): 351-359. 
117. Tinney, W. F., V. Brandwajn, and S. M. Chan. "Sparse vector methods." IEEE transactions on power apparatus and systems 2 (1985): 295-301.

118. Joskow, Paul, and Edward Kahn. "A quantitative analysis of pricing behavior in California's wholesale electricity market during summer 2000." In Power Engineering Society Summer Meeting, 2001, vol. 1, pp. 392-394. IEEE, 2001.

119. Borenstein, Severin, James Bushnell, and Frank Wolak. Diagnosing market power in California's restructured wholesale electricity market. No. w7868. National Bureau of Economic Research, 2000.

120. Fang, R. S., and A. K. David. "Transmission congestion management in an electricity market." IEEE Transactions on Power Systems 14, no. 3 (1999): 877883.

121. Jamasb, Tooraj, and Michael Pollitt. "Electricity market reform in the European Union: review of progress toward liberalization \& integration." The Energy Journal (2005): 11-41.

122. Cheung, Kwok W., Payman Shamsollahi, David Sun, James Milligan, and Mike Potishnak. "Energy and ancillary service dispatch for the interim ISO New England electricity market." In Power Industry Computer Applications, 1999. PICA'99. Proceedings of the 21st 1999 IEEE International Conference, pp. 47-53. IEEE, 1999.

123. Wolak, Frank A., and Robert H. Patrick. The impact of market rules and market structure on the price determination process in the England and Wales electricity market. No. w8248. National Bureau of Economic Research, 2001. 
124. Cheung, Kwok W. "Ancillary service market design and implementation in North America: from theory to practice." In Electric Utility Deregulation and Restructuring and Power Technologies, 2008. DRPT 2008. Third International Conference on, pp. 66-73. IEEE, 2008.

125. Ott, Andrew L. "Experience with PJM market operation, system design, and implementation." IEEE Transactions on Power Systems 18, no. 2 (2003): 528534.

126. Tong, Jianzhong. "Overview of PJM energy market design, operation and experience." In Electric Utility Deregulation, Restructuring and Power Technologies, 2004.(DRPT 2004). Proceedings of the 2004 IEEE International Conference on, vol. 1, pp. 24-27. IEEE, 2004.

127. Zareipour, Hamidreza, Claudio A. Cañizares, and Kankar Bhattacharya. "The operation of Ontario's competitive electricity market: overview, experiences, and lessons." IEEE Transactions on Power Systems 22, no. 4 (2007): 1782-1793.

128. Woo, Chi-Keung, Debra Lloyd, and Asher Tishler. "Electricity market reform failures: UK, Norway, Alberta and California." Energy policy 31, no. 11 (2003): 1103-1115.

129. Tinney, W. F., J. M. Bright, K. D. Demaree, and B. A. Hughes. "Some deficiencies in optimal power flow." IEEE Transactions on Power Systems 3, no. 2 (1988): 676-683.

130. Hao, Shangyou, and Alex Papalexopoulos. "External network modeling for optimal power flow applications." IEEE transactions on power systems 10, no. 2 (1995): 825-837. 
131. Nepomuceno, L., and A. Santos. "Equivalent optimization model for loss minimization: a suitable analysis approach." IEEE transactions on power systems 12, no. 4 (1997): 1403-1412.

132. Tognete, A. L., L. Nepomuceno, and A. dos Santos. "Framework for analysis and representation of external systems for online reactive-optimisation studies." IEE Proceedings-Generation, Transmission and Distribution 152, no. 6 (2005): $755-762$.

133. Shi, Di. "Power system network reduction for engineering and economic analysis." PhD diss., Arizona State University, 2012.

134. Cheng, Xu, and Thomas J. Overbye. "PTDF-based power system equivalents." IEEE Transactions on Power Systems 20, no. 4 (2005): 1868-1876.

135. R. Zimmerman, C. E. Murillo-Sanchez, and D. Gan, MATPOWER: A MATLAB Power System Simulation Package. Ithaca, NY, 2008.[Online]. Available: http://www.pserc.cornell.edu/matpower/.

136. NERC glossary of terms. Available at www.nerc.com/files/glossary_of terms.pdf accessed on January 2016

137. Liu, Minghai, and George Gross. "Effectiveness of the distribution factor approximations used in congestion modeling." In Proceedings of the 14th Power Systems Computation Conference, Seville, 24-28 June 2002. 2002.

138. Oren, Shmuel S., and Andrew M. Ross. "Economic congestion relief across multiple regions requires tradable physical flow-gate rights." IEEE Transactions on Power Systems 17, no. 1 (2002): 159-165. 
139. Hobbs, Benjamin F., Carolyn B. Metzler, and J-S. Pang. "Strategic gaming analysis for electric power systems: An MPEC approach." IEEE transactions on power systems 15 , no. 2 (2000): 638-645.

140. Chao, Hung-po, Stephen Peck, Shmuel Oren, and Robert Wilson. "Flowbased transmission rights and congestion management." The Electricity Journal 13 , no. 8 (2000): $38-58$.

141. Oh, HyungSeon. "A new network reduction methodology for power system planning studies." IEEE Transactions on Power Systems 25, no. 2 (2010): 677684.

142. Oh, HyungSeon. "Aggregation of buses for a network reduction." IEEE Transactions on Power Systems 27, no. 2 (2012): 705-712.

143. Shi, Di, Daniel L. Shawhan, Nan Li, Daniel J. Tylavsky, John T. Taber, Ray D. Zimmerman, and William D. Schulze. "Optimal generation investment planning: Pt. 1: network equivalents." In North American Power Symposium (NAPS), 2012, pp. 1-6. IEEE, 2012.

144. Shi, Di, and Daniel J. Tylavsky. "An improved bus aggregation technique for generating network equivalents." In 2012 IEEE Power and Energy Society General Meeting, pp. 1-8. IEEE, 2012.

145. Allen, Eric H., Jeffrey H. Lang, and Marija D. Ilic. "A combined equivalenced-electric, economic, and market representation of the northeastern power coordinating council us electric power system." IEEE Transactions on Power Systems 23 , no. 3 (2008): 896-907. 
146. Shi, Di, and Daniel J. Tylavsky. "A novel bus-aggregation-based structurepreserving power system equivalent." IEEE Transactions on Power Systems 30, no. 4 (2015): 1977-1986.

147. Singh, H. K., and S. C. Srivastava. "A reduced network representation suitable for fast nodal price calculations in electricity markets." In IEEE Power Engineering Society General Meeting, 2005, pp. 2070-2077. IEEE, 2005.

148. Akai, Terrence J., and Terence J. Akai. Applied numerical methods for engineers. No. 511 A33 1994. J. Wiley, 1994., pp. 171-177

149. Gubina, Ferdinand, and B. Strmenik. "Voltage collapse proximity index determination using voltage phasors approach." IEEE transactions on power systems 10, no. 2 (1995): 788-794.

150. PSSE Program Application Guide: Volume 1 pp. 8-2 accessed on January 2016

151. https://www.ee.washington.edu/research/pstca/ accessed August 2015

152. http://www.ee.washington.edu/research/pstca/pf300/pg_tca300fig.htm accessed on January 2016

153. http://al-roomi.org/power-flow/300-bus-system accessed on January 2016

154. Manitoba Hydro Electricity Board Annual Report 2014/15 issued by Manitoba Hydro. Available on line at https://www.hydro.mb.ca/corporate/ar/2014/annual_report_2014.shtml accessed on January 2016.

155. NERC white paper on "System Operating Limit Definition and Exceedance". Available on the online at 
http://www.nerc.com/pa/Stand/Prjct201403RvsnstoTOPandIROStndrds/2014 03

first_posting_white_paper_sol_exceedance_20140509.pdf accessed on January 2016.

156. Wijeweera, Gayan, Udaya D. Annakkage, Wenjie Zhang, and Athula D. Rajapakse. "Derivation of an equivalent circuit for real-time security assessment." IET Generation, Transmission \& Distribution 10, no. 8 (2016): 1913-1920.

157. C. L. Lawson, R. J. Hanson, "Solving least squares problems," Englewood Cliffs, NJ: Prentice-hall, vol. 161, 1974.

158. J. W. Demmel, "Applied numerical linear algebra” Siam, 1997 


\title{
Appendix A
}

\section{PSSE file used to modify three area 300 bus system}

\author{
text $* *$ \\ text $* *$ \\ BAT_SPLT,664,731,'731', 345 \\ BAT_SPLT,731,732,'732', 345 \\ BAT_SPLT,732,733,'733', 345 \\ BAT_SPLT,733,734,'734', 345 \\ BAT_SPLT,235,711,'711', 138 \\ BAT SPLT,711,712,'712', 138 \\ BAT_SPLT,712,713,'713', 138 \\ BAT_SPLT,713,714,'714', 138 \\ BAT_SPLT,714,715,'715', 138 \\ BAT_SPLT,250,761,'761', 66 \\ BAT_SPLT,761,762,'762', 66 \\ BAT_SPLT,281,721,'721', 230 \\ BAT_SPLT,721,722,'722', 230
}


BAT_SPLT,722,723,'723', 230

BAT_SPLT,721,724,'724', 230

BAT_SPLT,724,725,'725', 230

rdch

1

0 / END OF BUS DATA, BEGIN LOAD DATA

$731,1,1,,, 70,10$

$733,1,1,, 160,8$

$734,1,1,, 15,5$

$711,1,1,,, 35,15$

$712,1,1,, 15,3$

$713,1,1,,, 18,6$

$714,1,1,, 10,2$

$761,1,1,, 10,5$

$721,1,1,,, 40,8$

$723,1,1,,, 50,7$

0 / END OF LOAD DATA, BEGIN Fixed Shunt DATA

0 / END OF Fixed shunt DATA, BEGIN GENERATOR DATA

$732,1,200,40,100,-100,1.035,0,,,,,, 1$

$713,1,60,10,30,-30,1.035,0,,,,,,, 1$

$725,1,50,15,30,-30,1.04,0,,,,,,, 1$

$724,1,50,15,30,-30,1.04,0,,,{ }_{, \prime}, 1$

$715,1,60,10,50,-50,1.035,0,,,,,, 1$ 
0 / END OF GENERATOR DATA, BEGIN BRANCH DATA

$731,664,{ }^{\prime} 1$ ' $, \quad 0.31000 \mathrm{E}-02, \quad 0.28600 \mathrm{E}-01, \quad 0$

$731,732,,^{\prime} 1 ', \quad 0.35000 \mathrm{E}-02, \quad 0.3300 \mathrm{E}-01, \quad 0$.

$732,733,{ }^{\prime} 1$ ', $\quad 0.31000 \mathrm{E}-02, \quad 0.28600 \mathrm{E}-01, \quad 0$

$734,733,{ }^{\prime} 1$ ' $, \quad 0.5000 \mathrm{E}-02, \quad 0.4200 \mathrm{E}-01, \quad 0$

$235,711,{ }^{\prime} 1$ ', $\quad 0.5000 \mathrm{E}-03, \quad 0.177 \mathrm{E}-01, \quad 0$.

$712,711,{ }^{\prime} 1$ ' $, \quad 0.1000 \mathrm{E}-02, \quad 0.295 \mathrm{E}-01, \quad 0$

$712,713,{ }^{\prime} 1 ', \quad 0.3000 \mathrm{E}-03, \quad 0.13 \mathrm{E}-02, \quad 0$

$713,714,{ }^{\prime} 11^{\prime}, \quad 0.13 \mathrm{E}-02, \quad 0.288 \mathrm{E}-01, \quad 0.0$

$714,715,,^{\prime} 1, \quad 0.82000 \mathrm{E}-02, \quad 0.85 \mathrm{E}-01, \quad 0.02$

$250,761,{ }^{\prime} 1 ', \quad 0.302, \quad 0.446, \quad 0$

$761,762,{ }^{\prime} 1$ ', $\quad 0.24, \quad 0.421, \quad 0$

$281,721,{ }^{\prime} 1 ', \quad 0.44 \mathrm{E}-02, \quad 0.514 \mathrm{E}-01, \quad 0.0$

$722,721,{ }^{\prime} 1 ', \quad 0.11 \mathrm{E}-01, \quad 0.568 \mathrm{E}-01, \quad 0.0$

722,723,'1 ', $\quad 0.137 \mathrm{E}-01, \quad 0.957 \mathrm{E}-01, \quad 0.01$

$724,721,1^{\prime} 1, \quad 0.218-01, \quad 0.1511 \mathrm{E}-1, \quad 0.02$

$724,725,{ }^{\prime} 1$ ', $\quad 0.00754, \quad 0.0773, \quad 0.01$

$161,725,{ }^{\prime} 1 ', \quad 0.0489, \quad 0.3492, \quad 0.538$

0 / END OF BRANCH DATA, BEGIN TRANSFORMER DATA

$734,724,1$ 
$762,712,1$

$722,715,, 1$

0 / END OF TRANSFORMER DATA, BEGIN AREA DATA

0 / END OF AREA DATA, BEGIN TWO-TERMINAL DC DATA

0 / END OF TWO-TERMINAL DC DATA, BEGIN VSC DC LINE DATA

0 / END OF VSC DC LINE DATA, BEGIN SWITCHED SHUNT DATA

0 / END OF SWITCHED SHUNT DATA, BEGIN IMPEDANCE CORRECTION DATA

0 / END OF IMPEDANCE CORRECTION DATA, BEGIN MULTI-TERMINAL DC DATA

0 / END OF MULTI-TERMINAL DC DATA, BEGIN MULTI-SECTION LINE DATA 0 / END OF MULTI-SECTION LINE DATA, BEGIN ZONE DATA

0 / END OF ZONE DATA, BEGIN INTER-AREA TRANSFER DATA

0 / END OF INTER-AREA TRANSFER DATA, BEGIN OWNER DATA

0 / END OF OWNER DATA, BEGIN FACTS DEVICE DATA

0 / END OF FACTS DEVICE DATA 
chng

1

732

1

2

$-1$

713

1

2

$-1$

725

1

2

$-1$

724

1

2

$-1$

715

1

2

$-1$

$-1$ 
chng

1

161

1

1

0

$-1$

281

1

1

0

$-1$

$-1$

text $* *$ turn machine buses to code 2

text $* *$

text $* *$ 